\title{
6. A NEW LATE NEOGENE TIME SCALE: APPLICATION TO LEG 138 SITES ${ }^{1}$
}

\author{
N.J. Shackleton, ${ }^{2}$ S. Crowhurst, ${ }^{2}$ T. Hagelberg, ${ }^{3}$ N.G. Pisias, ${ }^{3}$ and D.A. Schneider ${ }^{4}$
}

\begin{abstract}
The sediments recovered during Leg 138 provide a remarkable opportunity to improve the geological time scale of the late Neogene. We have developed new time scales in the following steps. First, we constructed age models on the basis of shipboard magnetostratigraphy and biostratigraphy, using the time scale of Berggren, Kent, and Flynn (1985). Second, we refined these age models using shipboard GRAPE density measurements to provide more accurate correlation points. Third, we calibrated a time scale for the past 6 m.y. by matching the high-frequency GRAPE density variations to the orbital insolation record of Berger and Loutre (1991); we also took into account $\delta^{18} \mathrm{O}$ records, where they were available. Fourth, we generated a new seafloor anomaly time scale using our astronomical calibration of C3A.n (t) at $5.875 \mathrm{Ma}$ and an age of $9.639 \mathrm{Ma}$ for C $5 n$. $\ln (\mathrm{t})$ that is based on a new radiometric calibration (Baksi, 1992). Fifth, we recalibrated the records older than $6 \mathrm{Ma}$ to this new scale. Finally, we reconsidered the 6- to 10-Ma interval and found that this could also be partially tuned astronomically.
\end{abstract}

\section{INTRODUCTION}

In geology, the phrase "time scale" denotes the formal framework that is used to assign ages to geological deposits or to events in the geological record. It is often hard for a nongeologist to appreciate either the importance of the development of geological time scales or the difficulties that arise in generating and applying them. In this chapter, we focus on three types of "time scale." First, we have a time scale for variations in the geometry of the Earth-sun orbital system. We have used that published by Berger and Loutre (1991). Berger (1988) reviewed the history of studies of the Milankovitch theory in relation to climate in the geological past, and Berger and Loutre (1992) reviewed the accuracy of recent computations. Second, we generate a time scale for variations in sediment density (reflecting changes in the ratio of opal to calcite) that is based primarily on Sites 849,850 , and 851 , with records from Sites 846 and 847 providing important information; this time scale will probably be applicable to a large area of the equatorial Pacific Ocean. Third, we use this to recalibrate a section of the magnetic polarity time scale that is used globally to assign ages to rock sequences for recording an identified sequence of magnetic field reversals.

In another chapter (Shackleton et al., this volume), we use the time scale of this study to calibrate part of the oxygen isotope time scale (Shackleton and Opdyke, 1973; Imbrie et al., 1984). Finally, we apply our new time scale to the extensive series of biostratigraphic datums, determined by our colleagues, to refine the Neogene biostratigraphic time scale (Shackleton et al., biostratigraphic summary, this volume). The results of major synthetic studies on the geological time scale (Berggren, Kent, and Flynn [1985] and Berggren, Kent, and Van Couvering [1985]; Harland et al. [1990]) are usually presented in terms of age calibration of chronostratigraphic boundaries defined in stratotype sections. The geological literature is muddled by the fact that the word "age" has a specialized meaning: "sensu stricto the chronostratic division of rank between epoch and chron. .." (quoted from the glossary in Harland, 1978) that we do not make use of here. In this chapter, we are concerned with the numerical ages expressed in an astronomical unit (years) and calibrated through slower astronomical cycles.

${ }^{1}$ Pisias, N.G., Mayer, L.A., Janecek, T.R., Palmer-Julson, A., and van Andel, T.H. (Eds.), 1995. Proc. ODP, Sci. Results, 138: College Station, TX (Ocean Drilling Program).

${ }^{2}$ University of Cambridge, Godwin Laboratory, Free School Lane, Cambridge, CB2 3RS, United Kingdom.

${ }^{3}$ College of Oceanography, Oregon State University, Corvallis, OR 87331, U.S.A.

${ }^{4}$ Woods Hole Oceanographic Institution, Woods Hole, MA 02543, U.S.A.
The first statistically convincing demonstration that the imprint of variations in Earth's orbital geometry can be detected in deep-sea sediment records of climatic variability was that of Hays et al. (1976). The major advance that led to this work was the application of a reliable initial time scale through the simultaneous application of magnetostratigraphy and oxygen isotope stratigraphy in equatorial Pacific Ocean Core V28-238 (Shackleton and Opdyke, 1973), and indeed, preliminary spectral analysis indicated that the validation of the Milankovitch hypothesis was imminent. The advantage of the cores examined by Hays et al. (1976) was the relatively high sedimentation rate of about $4 \mathrm{~cm} / \mathrm{k} . \mathrm{y}$., which ensured that the evidence for precession could be detected. By contrast, Core V28-238, having a sedimentation rate of less than $2 \mathrm{~cm} / \mathrm{k} . \mathrm{y}$., barely preserves a precession signal.

Imbrie et al. (1984) published a time scale for the past 800 k.y. on the basis of a stack of oxygen isotope records from a number of cores. The major part of this calibration has held up to subsequent scrutiny, but the lowest part, which was dependent on two cores having low sedimentation rates, has undergone major revision (Shackleton et al., 1990; henceforth, SBP90). This revision was only possible because the Ocean Drilling Program (ODP) visited DSDP Site 504 again and resampled it as Site 677 using the advanced piston corer (APC). Site 677 has a consistent sedimentation rate of about $4 \mathrm{~cm} / \mathrm{k} . \mathrm{y}$. There is little doubt that sedimentation rate is the chief limitation on the reliable detection of orbital signals in deep-sea sequences. Leg 138 was planned so as to core a number of sites in the high productivity area of the eastern equatorial Pacific Ocean, where scientists already knew that high sedimentation rates could be anticipated, and where pervasive evidence of lithological cyclicity was also known (van Andel et al., 1975). Thus, an excellent opportunity was presented for extending the astronomical time scale.

\section{PAST RESEARCH}

The first steps toward astronomical calibration of the pre-Brunhes time scale were those of Pisias and Moore (1981), who had access to only relatively low-resolution data from a piston core. A major advance was made by Ruddiman et al. (1986) and Raymo et al. (1989), while working on DSDP Site 607 in the North Atlantic Ocean. These scientists showed that a long interval, now known to extend at least to $3 \mathrm{Ma}$, existed during which climatic variability was concentrated at the frequency of changes in obliquity (period 41 k.y.). Making only minor adjustments to the time scale based on linear interpolation between observed magnetic reversals, and using published ages for the last few reversals of Earth's magnetic field, these researchers developed a time scale that extended to about $2.4 \mathrm{Ma}$. This major 
achievement was possible because of the careful work that had been done to develop a complete and continuous section for Site 607 (Ruddiman, Kidd, Thomas, et al., 1987) and by the large amount of laboratory work that had been invested in that site.

Both Hilgen (1991a, henceforth H91) and SBP90 found evidence that this pioneering work, in fact, had led to incorrect conclusions. Hilgen's work was focused on the sequence of sapropels preserved in Pliocene rocks in southern Italy; he obtained an astronomically calibrated age for the Matuyama/Gauss boundary on the basis of matching these sequences with the astronomical eccentricity and precession signals. Soon after, Hilgen (1991b, henceforth H91) extended his calibration to the base of the Pliocene and gave ages for magnetic reversals back to Thvera Subchron (H91). SBP90 worked on planktonic and benthic $\delta^{18} \mathrm{O}$ records from ODP Site 677 in the eastern equatorial Pacific Ocean, covering the past 2.6 m.y. These workers identified three points where Ruddiman et al. (1986) had interpreted as a single obliquity cycle a section of record that actually spanned two obliquity cycles. Other researchers subsequently have confirmed this interpretation by examining GRAPE density records from the Atlantic (Herbert et al., 1992) and through a new high-resolution $\delta^{18} \mathrm{O}$ record from the Indian Ocean (Bassinot, pers. comm., 1992). Since that time, a number of scientists have provided new estimates of the age of the last few magnetic reversals (largely based on high precision ${ }^{40} \mathrm{Ar} /{ }^{39} \mathrm{Ar}$ dating) that support the new calibrations. For the Brunhes/Matuyama boundary, Izett and Obradovich (1991), Tauxe et al. (1992), Spell and McDougall (1992), Baksi et al. (1992), and Hall and Farrell (1993) all obtained ages supporting the new astronomically calibrated age of $0.78 \mathrm{Ma}$. For the Jaramillo Subchron, Glass et al. (1991), Spell and McDougall (1992), and Tauxe et al. (1992) found support for the new age (although Obradovich and Izett [1992] obtained values nearer the conventional age). Obradovich and Izett (1992) obtained age estimates for Cobb Mountain and for the base of the Olduvai to support the astronomical calibration. Walter et al. (1991) also obtained an age within the Olduvai Subchron at Olduvai Gorge to support the astronomical calibration of the base of the normal subchron. Walter et al. (1992) obtained ages in the Gauss that support the new calibration, and McDougall et al. (1992) showed that age determinations in the Gilbert (that had previously appeared anomalous), in fact, were in good agreement with the H91 time scale for the early Pliocene. Finally, Wilson (1993) demonstrated that if seafloor spreading rates are examined with high precision, they prove to be less variable when estimated using the astronomical time scale than when using other published time scales.

\section{MAGNETOSTRATIGRAPHY OF LEG 138 SITES}

The time scale used during Leg 138 was based on the version of the seafloor spreading magnetic anomaly time scale derived by Berggren, Kent, and Flynn (1985). In turn, this represented a new age calibration of the anomaly sequence created by LaBrecque et al. (1977), which was based on the classic South Atlantic profile of Heirtzler et al. (1968). Berggren, Kent, and Flynn (1985) used as age control points eight anomalies having ages that range from 3.40 to $84.0 \mathrm{~m} . \mathrm{y}$; they assumed linear spreading on their profile between these controls. Recently, Cande and Kent (1992) (henceforth CK92) introduced three significant modifications to this time scale. First, they generated a more reliable baseline anomaly sequence by re-evaluating a suite of South Atlantic profiles, instead of relying on the single profile of Heirtzler et al. (1968). Second, they restacked high-resolution profiles from other areas onto this improved South Atlantic sequence. Third, they used a cubic-spline, instead of a linear interpolation, to estimate the ages of anomalies between their calibration points; this is important from a geophysical standpoint, because it avoids introducing artificial instantaneous plate accelerations at the control point ages. CK92 also documented some additional reversals that were not included in the scheme of LaBrecque et al. (1977). Finally, CK92 introduced a minor improvement to the nomenclature, which we use in this chapter, alongside the familiar Pliocene-Pleistocene terminology. We have used the standard
South Atlantic Ocean profile of CK92 as our guide to the relative spatial and temporal spacing of reversals during the Neogene.

Among the 11 sites drilled during Leg 138 , eight $(844,845,848$, $850,851,852,853$, and 854 ) provided segments of useful magnetostratigraphy. Taken together, these provide a complete coverage of the polarity transitions of the last $13 \mathrm{~m} . \mathrm{y}$. since C5AB.n (t). For the purpose of calibration, reversals located in sediments having a higher sedimentation rate are more valuable. In this sense, Site 851 is particularly valuable for events between the present and C3n. In (the Cochiti Subchron). Site 852 preserves a good record to the top of C5.2n, except for the interval between C3A.nl and C4A.n1, where we rely on Site 853 . Site 848 also preserves a record to the base of C5r.1n. For the oldest part of the record, we rely primarily on Site 845, which extends to C5AB.n ( $t$ ). The depths of the reversals in each site are given in the appropriate site chapter, with a few exceptions. Schneider (this volume) has reinterpreted the data for Core 138$844 \mathrm{C}-6 \mathrm{H}$; we have accepted this new version. Schneider (this volume) has also improved the data from Site 845 by analyzing discrete samples. Again, we have used this revised data set. We accept the Gauss/Gilbert boundary in Hole 850B (plotted in error, p. 841, Fig. 22 of Mayer, Pisias, Janecek, et al., 1992). Data for Hole 851D are provided by Meynadier et al. (this volume).

\section{BIOSTRATIGRAPHY}

Remarkably high resolution was achieved in the shipboard biostratigraphy for all the major microfossil groups. Initially, age models were developed on the basis of the compilation given in the "Explanatory Notes" chapter (Shipboard Scientific Party, 1992). A small number of datum levels were redated (within the framework of the Berggren, Kent, and Flynn [1985] time scale) on the basis of the excellent magnetostratigraphy in Sites 844 and 845 . This more-or-less selfconsistent set of datum levels provided the basis for the age models developed in the site chapters and in Shackleton et al. (1992).

The objective of this chapter is to develop a more accurate time scale than has hitherto been available, by using the obvious cyclic character of the GRAPE density records as a monitor of the response of the fertile equatorial circulation system to forcing by variations in Earth's orbital geometry. The gamma-ray attenuation porosity evaluator (GRAPE) density tool is used aboard JOIDES Resolution to obtain automatic high-resolution records of sediment density; these data are discussed in Hagelberg et al. (this volume). In this region, sediment density varies with carbonate content, which in turn is closely linked to surface productivity. Since it was obvious at an early stage that this study would entail significant changes to the time scale used aboard JOIDES Resolution, biostratigraphic datum levels were used mainly to maintain the stratigraphic correlation between sites as the "tuning" was performed. Procedurally, this was done by recalculating the age for each datum level as the ages of the magnetostratigraphic boundaries were estimated again. Subsequently (Shackleton et al., this volume), we created a new set of best estimates for the ages of all useful biostratigraphic datums, based on the combined evidence of all the Leg 138 sites. Here, it is appropriate to remark that published estimates for a good proportion of the datums used were based on sparse data. This means that it is difficult to evaluate an age model for many of the sites because of apparent conflicts among age estimates suggested by data from different fossil groups. Ultimately, the most rigorous test of our age models will come, on the one hand, from the statistical evaluation of the patterns of density variability that they predict (Hagelberg et al., this volume) and, on the other, from further radiometric dating of the magnetic reversal sequence.

\section{OXYGEN ISOTOPE STRATIGRAPHY}

To maintain internal consistency in this study, we have attempted to develop a time scale that is based almost entirely on characteristic events in the GRAPE density records. We have not directly used the 
standard $\delta^{18} \mathrm{O}$ chronology in the upper part. As these data emerged, we have had access to the benthic $\delta^{18} \mathrm{O}$ records of Sites 846 and 849 (Mix et al., this volume) and to the planktonic $\delta^{18} \mathrm{O}$ records of Sites 847 (Farrell et al., this volume) and 851 (Ravelo et al., this volume) for the Pleistocene, as well as to the benthic $\delta^{18} \mathrm{O}$ record of Site 846 (Shackleton et al., this volume) for the Pliocene. Our aim has been to generate a GRAPE-based time scale for the Pleistocene that would not be in conflict with a $\delta^{18} \mathrm{O}$-based time scale, where that is available. Thus, we have used as control points features that are visible in the GRAPE density records. Initially, we utilized the same procedure throughout, correlating GRAPE density maxima to insolation maxima and GRAPE density minima to insolation minima. However, Farrell et al. (this volume) show that in the Pleistocene section of Site 847 , age differences between our time scale based on GRAPE density and one based on $\delta^{18} \mathrm{O}$ stratigraphy do arise, although they seldom exceed a few thousand years. Thus, we have in addition developed modified time scales for the past million years in which the ages for GRAPE density events have been shifted away from the ages of insolation maxima and minima to generate time scales that are closer to those suggested by the $\delta^{18} \mathrm{O}$ data.

In Tables 1 to 11 , we present age models that are probably close to a true $\delta^{18} \mathrm{O}$ time scale through the past million years; we also present (Table 12) the alternative age models for the upper part that were developed independent of the $\delta^{18} \mathrm{O}$ data. These may be regarded as viable alternative age models. Although age models based solely on GRAPE density might be expected to be less reliable than those based on $\delta^{18} \mathrm{O}$ stratigraphy, one cannot assume that the current $\delta^{18} \mathrm{O}$ time scale is perfect in every detail.

\section{TUNING METHODS}

It was a strength of the investigation by Hays et al. (1976) that they were able to document variance in the bandwidth of each of the three orbital variables (eccentricity, obliquity, and precession) in three independent paleoclimate proxies $\left(\delta^{18} \mathrm{O}\right.$, radiolarian-based sea-surface temperature, and percentage Cycladophora davisiana) using an age model for their cores that was entirely independently generated. In general, this is difficult to achieve, especially in a situation such as the eastern equatorial Pacific Ocean, where sedimentation rate clearly varies with climate, perhaps over a wide range. Thus, we have not attempted to demonstrate independently for each segment of time in each site that a statistical likelihood exists for the variability observed to be associated with orbital forcing. However, spectra on untuned sections of GRAPE density record consistently suggest concentration of power at orbital frequencies.

In a similar manner, Imbrie et al. (1984) did not attempt to demonstrate in advance that each of the records they used in their compilation contained the orbital imprint. Instead, they reasoned that the time scale that they generated gave rise to a sufficiently high coherency between $\delta^{18} \mathrm{O}$ and orbital insolation that the time scale was probably largely correct. Procedurally, Imbrie et al. (1984) used a strategy based on digital filters. To do this, one must develop an initial time scale, filter one orbital bandwidth (for example, obliquity), and note such small changes in the age model as may be needed to maintain a constant phase relationship between the filtered signal and the calculated obliquity record. The disadvantage of the method is that it is difficult to apply if sedimentation rates are extremely variable. In the case of the Leg 138 sites, it was already clear that this is so (Shackleton et al., 1992). For this reason, we chose to work entirely in the time domain, comparing GRAPE density with a target record derived from the orbital data.

This was also the strategy used by SBP90 for Site 677 (indeed the same strategy also had its place in the study of Imbrie et al. [1984] as considerable uncertainty regarding the appropriate "first guess" chronology existed when that work started). However, in the case of the work on Site 677, a reasonable tuning target already existed; SBP90 used the simple ice sheet model of Imbrie and Imbrie (1980) to
Table 1. Age model for Site 844 .

\begin{tabular}{|c|c|c|c|}
\hline $\begin{array}{l}\text { Age } \\
(\mathrm{Ma})\end{array}$ & $\begin{array}{l}\text { Depth } \\
\text { (mcd) }\end{array}$ & $\begin{array}{l}\text { Age } \\
\text { (Ma) }\end{array}$ & $\begin{array}{l}\text { Depth } \\
\text { (mcd) }\end{array}$ \\
\hline 0.000 & 0.00 & 4.981 & 29.90 \\
\hline 0.088 & 0.58 & 5.232 & 31.30 \\
\hline 0.127 & 1.48 & 5.875 & 35.10 \\
\hline 0.140 & 1.63 & 6.122 & 36.85 \\
\hline 0.174 & 2.07 & 6.256 & 37.85 \\
\hline 0.184 & 2.35 & 6.554 & 38.70 \\
\hline 0.247 & 3.27 & 6.919 & 41.00 \\
\hline 0.354 & 4.39 & 7.072 & 41.80 \\
\hline 0.479 & 6.43 & 7.406 & 43.15 \\
\hline 0.509 & 6.78 & 7.533 & 44.40 \\
\hline 0.579 & 7.76 & 7.618 & 44.95 \\
\hline 0.614 & 8.43 & 8.027 & 49.95 \\
\hline 0.628 & 8.50 & 8.631 & 53.72 \\
\hline 0.659 & 8.80 & 8.945 & 56.75 \\
\hline 0.717 & 9.31 & 9.142 & 58.00 \\
\hline 0.737 & 9.60 & 9.218 & 58.90 \\
\hline 0.783 & 10.09 & 9.482 & 60.60 \\
\hline 0.990 & 11.83 & 9.543 & 61.95 \\
\hline 1.070 & 12.63 & 9.639 & 62.40 \\
\hline 1.770 & 18.10 & 10.022 & 67.13 \\
\hline 1.950 & 19.15 & 10.548 & 73.01 \\
\hline 2.600 & 22.20 & 10.693 & 75.27 \\
\hline 3.053 & 23.75 & 10.991 & 81.74 \\
\hline 3.131 & 23.95 & 11.373 & 93.65 \\
\hline 3.224 & 24.40 & 11.988 & 116.77 \\
\hline 3.337 & 24.50 & 12.636 & 134.68 \\
\hline 3.611 & 25.45 & 12.929 & 148.04 \\
\hline 4.192 & 26.95 & 13.252 & 160.48 \\
\hline 4.322 & 27.40 & 14.070 & 189.45 \\
\hline 4.478 & 27.90 & 14.950 & 219.98 \\
\hline 4.604 & 28.40 & 15.830 & 258.05 \\
\hline 4.784 & 29.05 & 17.060 & 308.30 \\
\hline
\end{tabular}

Table 2. Age model for Site 845 .

\begin{tabular}{|c|c|c|c|}
\hline $\begin{array}{l}\text { Age } \\
\text { (Ma) }\end{array}$ & $\begin{array}{l}\text { Depth } \\
\text { (mcd) }\end{array}$ & $\begin{array}{l}\text { Age } \\
\text { (Ma) }\end{array}$ & $\begin{array}{l}\text { Depth } \\
\text { (mcd) }\end{array}$ \\
\hline 0.000 & 0.00 & 8.205 & 120.82 \\
\hline 0.410 & 12.32 & 8.631 & 129.71 \\
\hline 1.961 & 43.03 & 8.945 & 136.74 \\
\hline 3.053 & 53.88 & 9.142 & 140.01 \\
\hline 3.131 & 54.98 & 9.218 & 141.43 \\
\hline 3.224 & 55.98 & 9.482 & 145.08 \\
\hline 3.337 & 57.38 & 9.543 & 146.70 \\
\hline 3.611 & 59.88 & 9.639 & 147.74 \\
\hline 4,192 & 65.56 & 9.775 & 150.34 \\
\hline 4.322 & 66.62 & 9.815 & 150.78 \\
\hline 4.478 & 68.51 & 10.839 & 164.94 \\
\hline 4.604 & 69.76 & 10.943 & 166.03 \\
\hline 4.784 & 71.59 & 10.991 & 166.78 \\
\hline 4.878 & 72.04 & 11.373 & 174.17 \\
\hline 4.981 & 73.29 & 11.428 & 175.78 \\
\hline 5.232 & 75.97 & 11.841 & 183.65 \\
\hline 5.875 & 87.49 & 11.988 & 187.06 \\
\hline 6.122 & 90.22 & 12.605 & 204.91 \\
\hline 6.256 & 93.00 & 12.637 & 206.26 \\
\hline 6.555 & 96.31 & 12.705 & 208.13 \\
\hline 6.919 & 101.44 & 12.752 & 209.57 \\
\hline 7.072 & 103.18 & 12.929 & 215.54 \\
\hline 7.406 & 108.04 & 13.083 & 220.07 \\
\hline 7.533 & 110.12 & 13.252 & 226.07 \\
\hline 7.618 & 111.02 & 15.830 & 275.23 \\
\hline 8.027 & 119.29 & 16.450 & 309.10 \\
\hline 8.174 & 120.53 & & \\
\hline
\end{tabular}

produce a target record that embodied the same time constants relating ice volume and summer insolation at $65^{\circ} \mathrm{N}$ that have been documented for the late Pleistocene. In the case of GRAPE density (or the underlying variable, the ratio of calcite to biogenic opal), we do not have a model linking the forcing and the response. Therefore, we have simply used the calculated record of summer insolation at $65^{\circ} \mathrm{N}$ as the tuning target. We have throughout assumed that no phase lag existed between insolation and GRAPE density and that high density (high percentage of $\mathrm{CaCO}_{3}$ ) is associated with high Northern Hemisphere summer insolation. This phase relationship may be approximately valid for the most recent past; in the Pacific Ocean, a low percentage 
Table 3. Age Model for Site 846.

\begin{tabular}{|c|c|c|c|c|c|c|c|}
\hline $\begin{array}{l}\text { Age } \\
(\mathrm{Ma})\end{array}$ & $\begin{array}{l}\text { Depth } \\
\text { (mcd) }\end{array}$ & $\begin{array}{l}\text { Age } \\
(\mathrm{Ma})\end{array}$ & $\begin{array}{l}\text { Depth } \\
\text { (mcd) }\end{array}$ & $\begin{array}{l}\text { Age } \\
\text { (Ma) }\end{array}$ & $\begin{array}{l}\text { Depth } \\
\text { (mcd) }\end{array}$ & $\begin{array}{l}\text { Age } \\
(\mathrm{Ma})\end{array}$ & $\begin{array}{l}\text { Depth } \\
\text { (mcd) }\end{array}$ \\
\hline 0.000 & 0.00 & 2.023 & 74.88 & 4.154 & 161.02 & 6.404 & 262.79 \\
\hline 0.039 & 1.80 & 2.086 & 79.00 & 4.182 & 161.78 & 6.423 & 263.59 \\
\hline 0.088 & 3.70 & 2.097 & 79.50 & 4.204 & 162.34 & 6.443 & 263.91 \\
\hline 0.127 & 5.96 & 2.118 & 80.06 & 4.225 & 163.00 & 6.501 & 265.35 \\
\hline 0.148 & 6.76 & 2.140 & 80.74 & 4.246 & 163.60 & 6.535 & 266.61 \\
\hline 0.171 & 7.38 & 2.190 & 82.60 & 4.272 & 164.74 & 6.540 & 266.73 \\
\hline 0.184 & 7.94 & 2.211 & 83.88 & 4.296 & 165.50 & 7.080 & 280.34 \\
\hline 0.220 & 8.76 & 2.233 & 84.54 & 4.319 & 166.04 & 7.090 & 281.16 \\
\hline 0.240 & 9.60 & 2.255 & 85.56 & 4.339 & 167.08 & 7.117 & 282.60 \\
\hline 0.247 & 9.94 & 2.278 & 86.48 & 4.361 & 167.74 & 7.163 & 284.86 \\
\hline 0.278 & 11.04 & 2.305 & 87.48 & 4.412 & 169.66 & 7.204 & 286.98 \\
\hline 0.290 & 11.26 & 2.348 & 89.26 & 4.451 & 170.88 & 7.225 & 287.75 \\
\hline 0.310 & 11.84 & 2.377 & 90.16 & 4.491 & 172.02 & 7.315 & 290.05 \\
\hline 0.326 & 12.36 & 2.411 & 91.62 & 4.537 & 174.22 & 7.370 & 292.70 \\
\hline 0.333 & 12.66 & 2.422 & 92.22 & 4.562 & 175.26 & 7.404 & 294.54 \\
\hline 0.354 & 13.44 & 2.438 & 92.64 & 4.595 & 177.24 & 7.474 & 296.84 \\
\hline 0.388 & 15.04 & 2.477 & 94.94 & 4.629 & 179.20 & 7.496 & 297.34 \\
\hline 0.405 & 15.70 & 2.490 & 95.54 & 4.653 & 180.12 & 7.740 & 301.84 \\
\hline 0.422 & 16.26 & 2.521 & 96.52 & 4.676 & 181.26 & 7.878 & 304.84 \\
\hline 0.446 & 17.06 & 2.534 & 97.68 & 4.698 & 182.82 & 7.921 & 305.74 \\
\hline 0.479 & 18.78 & 2.547 & 98.78 & 4.722 & 183.78 & 7.946 & 306.39 \\
\hline 0.509 & 19.90 & 2.569 & 99.56 & 4.744 & 184.96 & 8.013 & 307.49 \\
\hline 0.515 & 20.14 & 2.592 & 100.36 & 4.770 & 186.64 & 8.080 & 308.74 \\
\hline 0.523 & 20.38 & 2.637 & 102.43 & 4.812 & 187.88 & 8.301 & 310.54 \\
\hline 0.544 & 20.96 & 2.683 & 104.43 & 4.866 & 190.14 & 8.312 & 310.79 \\
\hline 0.579 & 22.12 & 2.707 & 105.37 & 4.896 & 191.40 & 8.322 & 311.19 \\
\hline 0.614 & 22.88 & 2.741 & 107.27 & 4.919 & 192.38 & 8.373 & 312.24 \\
\hline 0.628 & 23.38 & 2.754 & 108.27 & 4.940 & 193.46 & 8.414 & 312.94 \\
\hline 0.659 & 24.42 & 2.776 & 109.39 & 4.984 & 194.64 & 8.434 & 313.29 \\
\hline 0.681 & 25.38 & 2.798 & 110.13 & 5.011 & 195.26 & 8.470 & 313.79 \\
\hline 0.692 & 25.72 & 2.835 & 111.61 & 5.033 & 195.73 & 8.547 & 314.59 \\
\hline 0.717 & 26.26 & 2.853 & 112.53 & 5.077 & 196.87 & 8.639 & 315.69 \\
\hline 0.737 & 26.92 & 2.876 & 113.63 & 5.101 & 197.41 & 8.682 & 316.29 \\
\hline 0.772 & 28.16 & 2.904 & 115.21 & 5.127 & 198.31 & 8.732 & 317.59 \\
\hline 0.783 & 28.74 & 2.926 & 115.83 & 5.148 & 199.41 & 8.776 & 318.29 \\
\hline 0.863 & 32.18 & 2.949 & 117.61 & 5.170 & 200.77 & 8.786 & 318.49 \\
\hline 0.884 & 33.26 & 2.994 & 119.57 & 5.224 & 202.53 & 8.833 & 318.64 \\
\hline 0.908 & 34.34 & 3.042 & 121.71 & 5.264 & 204.65 & 8.926 & 320.94 \\
\hline 0.936 & 35.14 & 3.063 & 122.55 & 5.300 & 206.79 & 8.990 & 322.65 \\
\hline 0.978 & 35.98 & 3.085 & 123.81 & 5.342 & 208.41 & 9.084 & 324.30 \\
\hline 0.999 & 36.56 & 3.135 & 125.49 & 5.392 & 210.07 & 9.133 & 325.10 \\
\hline 1.029 & 37.46 & 3.156 & 126.37 & 5.414 & 211.71 & 9.155 & 325.40 \\
\hline 1.050 & 37.92 & 3.177 & 127.05 & 5.435 & 212.57 & 9.199 & 326.60 \\
\hline 1.092 & 39.80 & 3.198 & 127.75 & 5.457 & 213.43 & 9.291 & 328.05 \\
\hline 1.114 & 40.80 & 3.231 & 128.49 & 5.507 & 215.83 & 9.420 & 330.15 \\
\hline 1.136 & 42.12 & 3.288 & 129.91 & 5.528 & 216.57 & 9.465 & 330.65 \\
\hline 1.215 & 44.68 & 3.328 & 131.39 & 5.549 & 217.97 & 9.489 & 331.05 \\
\hline 1.243 & 45.14 & 3.371 & 133.51 & 5.569 & 218.93 & 9.511 & 331.40 \\
\hline 1.317 & 47.12 & 3.401 & 134.51 & 5.587 & 220.21 & 9.535 & 331.75 \\
\hline 1.337 & 47.74 & 3.422 & 135.51 & 5.624 & 223.25 & 9.557 & 332.10 \\
\hline 1.358 & 48.86 & 3.464 & 138.05 & 5.643 & 224.15 & 9.626 & 333.00 \\
\hline 1.379 & 49.54 & 3,496 & 139.13 & 5.661 & 225.05 & 9.662 & 333.40 \\
\hline 1.400 & 50.64 & 3.516 & 139.59 & 5.680 & 226.03 & 9.704 & 333.85 \\
\hline 1.431 & 52.14 & 3.526 & 140.01 & 5.700 & 226.89 & 9.735 & 334.45 \\
\hline 1.473 & 53.06 & 3.578 & 142.99 & 5.721 & 227.23 & 9.754 & 334.70 \\
\hline 1.493 & 53.58 & 3.614 & 144.03 & 5.752 & 229.01 & 9.798 & 335.40 \\
\hline 1.513 & 54.58 & 3.654 & 145.27 & 5.772 & 230.79 & 9.822 & 335.90 \\
\hline 1.528 & 54.92 & 3.680 & 145.79 & 5.794 & 232.05 & 9.847 & 336.25 \\
\hline 1.547 & 55.22 & 3.709 & 146.69 & 5.815 & 235.61 & 9.892 & 336.74 \\
\hline 1.567 & 55.80 & 3.730 & 147.51 & 5.864 & 237.49 & 9.913 & 336.99 \\
\hline 1.606 & 57.24 & 3.751 & 147.99 & 5.887 & 238.31 & 9.985 & 338.29 \\
\hline 1.645 & 58.28 & 3.781 & 148.93 & 5.981 & 242.07 & 10.372 & 343.29 \\
\hline 1.664 & 59.18 & 3.802 & 149.48 & 6.060 & 247.42 & 10.693 & 346.54 \\
\hline 1.697 & 60.96 & 3.824 & 150.14 & 6.099 & 249.76 & 10.987 & 353.39 \\
\hline 1.708 & 61.72 & 3.867 & 151.32 & 6.174 & 252.88 & 11.212 & 357.04 \\
\hline 1.782 & 64.02 & 3.918 & 152.70 & 6.215 & 253.38 & 11.377 & 359.94 \\
\hline 1.832 & 65.88 & 3.960 & 154.30 & 6.267 & 255.14 & 13.326 & 394.97 \\
\hline 1.875 & 67.72 & 4.014 & 156.88 & 6.289 & 255.50 & 13.480 & 400.00 \\
\hline 1.926 & 70.24 & 4.034 & 157.46 & 6.309 & 255.92 & 15.835 & 423.80 \\
\hline 1.947 & 71.20 & 4.089 & 159.02 & 6.330 & 258.80 & 18.067 & 460.00 \\
\hline 1.968 & 72.36 & 4.110 & 159.52 & 6.349 & 260.12 & & \\
\hline 1.986 & 73.62 & 4.131 & 160.22 & 6.383 & 262.35 & & \\
\hline
\end{tabular}

of $\mathrm{CaCO}_{3}$ is associated with interglacial-to-glacial transitions and good carbonate preservation with glacial-to-interglacial transitions (e.g., Ninkovich and Shackleton, 1975; Keir and Berger, 1985; Le and Shackleton, 1992). Whether this phase relationship is appropriate to the equatorial high productivity belt in the eastern Pacific Ocean, and whether the same phase relationship is appropriate for the older part of the record, is not yet known. Although the uncertain phase contributes a significant source of potential error in our time scale, it only entails, at a maximum, a few thousand years of systematic error in the uncertainty of the age estimates.
Table 4. Age model for Site 847

\begin{tabular}{|c|c|c|c|c|c|}
\hline $\begin{array}{l}\text { Age } \\
\text { (Ma) }\end{array}$ & $\begin{array}{l}\text { Depth } \\
\text { (mcd) }\end{array}$ & $\begin{array}{l}\text { Age } \\
(\mathrm{Ma})\end{array}$ & $\begin{array}{l}\text { Depth } \\
\text { (mcd) }\end{array}$ & $\begin{array}{l}\text { Age } \\
\text { (Ma) }\end{array}$ & $\begin{array}{l}\text { Depth } \\
\text { (mcd) }\end{array}$ \\
\hline 0.000 & 0.00 & 2.211 & 73.33 & 4.110 & 129.10 \\
\hline 0.039 & 1.79 & 2.233 & 74.03 & 4.131 & 129.54 \\
\hline 0.088 & 3.01 & 2.255 & 74.99 & 4.154 & 130.14 \\
\hline 0.116 & 3.81 & 2.278 & 75.51 & 4.182 & 130.70 \\
\hline 0.127 & 4.11 & 2.305 & 76.13 & 4.204 & 131.28 \\
\hline 0.148 & 4.75 & 2.348 & 77.43 & 4.225 & 131.94 \\
\hline 0.171 & 5.99 & 2.390 & 78.91 & 4.246 & 132.52 \\
\hline 0.184 & 6.73 & 2.422 & 79.69 & 4.296 & 133.82 \\
\hline 0.220 & 7.45 & 2.438 & 80.39 & 4.319 & 134.40 \\
\hline 0.240 & 8.17 & 2.477 & 82.39 & 4.339 & 135.42 \\
\hline 0.247 & 8.83 & 2.521 & 83.57 & 4.361 & 136.00 \\
\hline 0.278 & 9.49 & 2.547 & 84.49 & 4.376 & 136.80 \\
\hline 0.290 & 9.79 & 2.569 & 84.85 & 4.412 & 137.76 \\
\hline 0.308 & 10.29 & 2.592 & 85.91 & 4.433 & 138.02 \\
\hline 0.326 & 10.77 & 2.614 & 86.77 & 4.491 & 139.88 \\
\hline 0.333 & 11.13 & 2.637 & 87.25 & 4.537 & 141.86 \\
\hline 0.349 & 11.81 & 2.683 & 88.83 & 4.562 & 143.44 \\
\hline 0.388 & 13.19 & 2.707 & 89.47 & 4.583 & 144.26 \\
\hline 0.405 & 13.77 & 2.729 & 89.89 & 4.606 & 146.68 \\
\hline 0.422 & 14.05 & 2.754 & 91.11 & 4.629 & 148.70 \\
\hline 0.446 & 14.51 & 2.776 & 91.47 & 4.653 & 151.60 \\
\hline 0.463 & 15.19 & 2.786 & 91.79 & 4.698 & 155.60 \\
\hline 0.479 & 15.71 & 2.798 & 92.45 & 4.722 & 156.70 \\
\hline 0.484 & 16.05 & 2.835 & 93.93 & 4.744 & 157.30 \\
\hline 0.509 & 16.69 & 2.876 & 94.83 & 4.770 & 158.70 \\
\hline 0.544 & 17.45 & 2.904 & 95.41 & 4.821 & 159.90 \\
\hline 0.579 & 18.23 & 2.926 & 96.33 & 4.847 & 161.80 \\
\hline 0.614 & 19.09 & 2.937 & 96.97 & 4.866 & 163.50 \\
\hline 0.636 & 19.59 & 2.949 & 97.23 & 4.896 & 166.20 \\
\hline 0.659 & 20.33 & 2.969 & 97.75 & 4.919 & 167.20 \\
\hline 0.681 & 21.27 & 2.994 & 98.31 & 4.940 & 167.80 \\
\hline 0.692 & 21.47 & 3.019 & 99.07 & 4.962 & 168.30 \\
\hline 0.717 & $22-23$ & 3.030 & 99.41 & 4.984 & 169.00 \\
\hline 0.737 & 22.69 & 3.041 & 99.69 & 5.033 & 170.80 \\
\hline 0.772 & 23.39 & 3.063 & 100.11 & 5.055 & 171.30 \\
\hline 0.783 & 24.29 & 3.085 & 100.63 & 5.077 & 172.50 \\
\hline 0.824 & 25.15 & 3.111 & 101.29 & 5.101 & 173.80 \\
\hline 0.908 & 28.15 & 3.135 & 102.12 & 5.107 & 174.10 \\
\hline 0.978 & 29.61 & 3.156 & 102.82 & 5.148 & 174.50 \\
\hline 1.000 & 30.43 & 3.177 & 103.42 & 5.170 & 176.60 \\
\hline 1.050 & 31.53 & 3.198 & 103.92 & 5.224 & 178.30 \\
\hline 1.072 & 32.67 & 3.231 & 104.66 & 5.245 & 179.30 \\
\hline 1.205 & 37.51 & 3.252 & 105.72 & 5.264 & 180.20 \\
\hline 1.215 & 38.15 & 3.271 & 106.28 & 5.300 & 181.70 \\
\hline 1.243 & 39.09 & 3.288 & 106.68 & 5.320 & 182.60 \\
\hline 1.272 & 39.77 & 3.308 & 107.48 & 5.342 & 183.70 \\
\hline 1.283 & 40.51 & 3.328 & 107.94 & 5.392 & 185.30 \\
\hline 1.307 & 41.07 & 3.348 & 108.68 & 5.414 & 185.90 \\
\hline 1.327 & 41.73 & 3.359 & 108.96 & 5.435 & 186.90 \\
\hline 1.337 & 42.41 & 3.391 & 109.72 & 5.507 & 189.50 \\
\hline 1.379 & 43.81 & 3.401 & 110.06 & 5.528 & 190.30 \\
\hline 1.400 & 44.75 & 3.422 & 110.80 & 5.549 & 191.10 \\
\hline 1.431 & 45.57 & 3.443 & 111.44 & 5.569 & 192.20 \\
\hline 1.493 & 47.55 & 3.464 & 111.84 & 5.587 & 192.80 \\
\hline 1.528 & 48.59 & 3.496 & 112.62 & 5.603 & 193.80 \\
\hline 1.567 & 49.83 & 3.516 & 113.10 & 5.624 & 194.60 \\
\hline 1.606 & 50.89 & 3.578 & 114.94 & 5.643 & 196.20 \\
\hline 1.697 & 53.09 & 3.604 & 115.74 & 5.680 & 198.50 \\
\hline 1.718 & 55.15 & 3.633 & 116.84 & 5.721 & 199.30 \\
\hline 1.750 & 55.87 & 3.654 & 117.38 & 5.752 & 200.10 \\
\hline 1.811 & 57.65 & 3.689 & 118.28 & 5.772 & 201.50 \\
\hline 1.832 & 58.79 & 3.720 & 119.40 & 5.783 & 202.00 \\
\hline 1.875 & 61.25 & 3.750 & 120.00 & 5.794 & 202.20 \\
\hline 1.904 & 61.93 & 3.824 & 122.14 & 5.815 & 204.10 \\
\hline 1.947 & 63.71 & 3.867 & 123.32 & 5.836 & 205.20 \\
\hline 1.958 & 64.67 & 3.896 & 124.10 & 5.864 & 206.00 \\
\hline 2.003 & 66.15 & 3.918 & 124.60 & 5.887 & 206.70 \\
\hline 2.023 & 66.83 & 3.939 & 125.06 & 5.909 & 207.50 \\
\hline 2.042 & 68.15 & 3.960 & 125.72 & 5.929 & 208.90 \\
\hline 2.052 & 68.59 & 4.014 & 126.84 & 6.003 & 211.00 \\
\hline 2.097 & 70.07 & 4.034 & 127.26 & 6.300 & 230.21 \\
\hline 2.118 & 70.55 & 4.055 & 127.98 & 6.700 & 251.01 \\
\hline 2.129 & 70.83 & 4.072 & 128.26 & & \\
\hline
\end{tabular}

Procedurally, the process of tuning the GRAPE density data may be idealized as follows. We must, however, emphasize that in reality a considerable number of iterations exist for each step. The work was performed on three-dimensional LOTUS 1-2-3 spreadsheets. For each site, the starting point was the composite section of continuous GRAPE density record generated by splicing segments of data from among the holes available and shown by Hagelberg et al. (1992; Chapter 5, Fig. 6) and by Shackleton et al. (1992; Chapter 6, Fig. 1). 
Table 5. Age model for Site 848.

\begin{tabular}{|c|c|c|c|}
\hline $\begin{array}{l}\text { Age } \\
\text { (Ma) }\end{array}$ & $\begin{array}{l}\text { Depth } \\
\text { (mcd) }\end{array}$ & $\begin{array}{l}\text { Age } \\
(\mathrm{Ma})\end{array}$ & $\begin{array}{l}\text { Depth } \\
\text { (mcd) }\end{array}$ \\
\hline 0.000 & 0.00 & 2.614 & 31.18 \\
\hline 0.039 & 0.68 & 2.637 & 31.34 \\
\hline 0.056 & 1.02 & 2.695 & 31.74 \\
\hline 0.088 & 1.44 & 2.766 & 32.36 \\
\hline 0.127 & 1.76 & 2.864 & 32.90 \\
\hline 0.171 & 2.46 & 2.892 & 33.18 \\
\hline 0.184 & 2.64 & 2.937 & 33.40 \\
\hline 0.247 & 3.12 & 2.969 & 33.60 \\
\hline 0.278 & 3.68 & 2.994 & 33.68 \\
\hline 0.290 & 3.80 & 3.030 & 33.86 \\
\hline 0.326 & 4.16 & 3.054 & 33.92 \\
\hline 0.349 & 4.70 & 3.231 & 34.52 \\
\hline 0.372 & 5.20 & 3.252 & 34.66 \\
\hline 0.388 & 5.52 & 3.318 & 34.96 \\
\hline 0.405 & 5.64 & 3.359 & 35.24 \\
\hline 0.422 & 5.96 & 3.433 & 35.64 \\
\hline 0.446 & 6.52 & 3.440 & 35.70 \\
\hline 0.479 & 7.20 & 3.475 & 35.82 \\
\hline 0.504 & 7.64 & 3.516 & 35.92 \\
\hline 0.526 & 7.88 & 3.537 & 36.02 \\
\hline 0.579 & 8.98 & 3.578 & 36.38 \\
\hline 0.614 & 9.30 & 3.654 & 36.80 \\
\hline 0.628 & 9.64 & 3.689 & 37.00 \\
\hline 0.659 & 10.36 & 3.730 & 37.16 \\
\hline 0.681 & 10.80 & 3.781 & 37.38 \\
\hline 0.717 & 11.34 & 3.867 & 37.64 \\
\hline 0.737 & 11.70 & 3.918 & 37.92 \\
\hline 0.772 & 12.20 & 4.110 & 38.68 \\
\hline 0.783 & 12.46 & 4.154 & 38.84 \\
\hline 0.863 & 13.80 & 4.204 & 39.12 \\
\hline 0.908 & 14.86 & 4.334 & 39.64 \\
\hline 0.925 & 15.16 & 4.361 & 39.84 \\
\hline 0.978 & 15.76 & 4.491 & 40.70 \\
\hline 0.999 & 16.02 & 4.537 & 41.04 \\
\hline 1.061 & 16.96 & 4.583 & 41.48 \\
\hline 1.092 & 17.84 & 4.606 & 42.08 \\
\hline 1.215 & 19.66 & 4.744 & 44.28 \\
\hline 1.272 & 20.60 & 4.770 & 44.80 \\
\hline 1.307 & 21.04 & 4.821 & 45.28 \\
\hline 1.347 & 21.48 & 4.847 & 45.70 \\
\hline 1.379 & 21.86 & 4.880 & 46.20 \\
\hline 1.400 & 22.44 & 5.004 & 47.64 \\
\hline 1.431 & 22.88 & 5.170 & 49.44 \\
\hline 1.493 & 23.50 & 5.224 & 50.40 \\
\hline 1.528 & 23.82 & 5.342 & 52.95 \\
\hline 1.567 & 24.28 & 5.528 & 56.45 \\
\hline 1.606 & 24.44 & 5.549 & 57.15 \\
\hline 1.664 & 24.96 & 5.680 & 59.85 \\
\hline 1.718 & 25.52 & 6.099 & 65.05 \\
\hline 1.750 & 25.70 & 6.330 & 67.65 \\
\hline 1.799 & 26.28 & 6.575 & 70.85 \\
\hline 1.821 & 26.50 & 7.406 & 81.05 \\
\hline 1.875 & 27.00 & 7.533 & 82.00 \\
\hline 1.916 & 27.30 & 7.618 & 82.25 \\
\hline 1.958 & 27.50 & 8.027 & 84.40 \\
\hline 2.003 & 27.74 & 8.174 & 84.70 \\
\hline 2.052 & 28.08 & 8.631 & 87.60 \\
\hline 2.086 & 28.42 & 8.945 & 89.70 \\
\hline 2.097 & 28.60 & 9.142 & 91.00 \\
\hline 2.140 & 28.76 & 9.218 & 91.95 \\
\hline 2.245 & 29.20 & 9.482 & 94.20 \\
\hline 2.293 & 29.32 & 9.543 & 94.60 \\
\hline 2.325 & 29.44 & 9.639 & 94.75 \\
\hline 2.377 & 29.82 & 9.775 & 95.20 \\
\hline 2.411 & 30.06 & 9.815 & 95.40 \\
\hline 2.438 & 30.14 & 10.839 & 102.80 \\
\hline 2.490 & 30.44 & 10.943 & 103.35 \\
\hline 2.500 & 30.52 & 10.991 & 103.65 \\
\hline 2.534 & 30.70 & 11.270 & 105.60 \\
\hline
\end{tabular}

For Sites 846 to 852 , this was later replaced by the stacked record, generated by Hagelberg et al. (this volume). This stacked record is based on the same depth scale as the original spliced record, with data from all the other holes at the site stacked onto it and averaged to provide a record having a higher signal-to-noise ratio. This GRAPE density record was placed first on a low-resolution time scale. This time scale was based on the age models in Shackleton et al. (1992) modified on the basis of a smooth conversion to the CK92 magnetostratigraphic time scale. The GRAPE density time series then was compared on the same age scale with the orbital insolation record.
Table 6. Age model for Site 849.

\begin{tabular}{|c|c|c|c|c|c|c|c|}
\hline $\begin{array}{l}\text { Age } \\
(\mathrm{Ma})\end{array}$ & $\begin{array}{l}\text { Depth } \\
\text { (mcd) }\end{array}$ & $\begin{array}{l}\text { Age } \\
\text { (Ma) }\end{array}$ & $\begin{array}{l}\text { Depth } \\
\text { (mcd) }\end{array}$ & $\begin{array}{l}\text { Age } \\
\text { (Ma) }\end{array}$ & $\begin{array}{l}\text { Depth } \\
\text { (mcd) }\end{array}$ & $\begin{array}{l}\text { Age } \\
\text { (Ma) }\end{array}$ & $\begin{array}{l}\text { Depth } \\
\text { (mcd) }\end{array}$ \\
\hline 0.000 & 0.02 & 2.521 & 71.10 & 4.513 & 126.32 & 6.554 & 261.41 \\
\hline 0.039 & 1.16 & 2.534 & 71.72 & 4.537 & 127.62 & 6.575 & 263.50 \\
\hline 0.088 & 2.76 & 2.547 & 71.98 & 4.562 & 129.62 & 6.596 & 265.60 \\
\hline 0.116 & 3.60 & 2.569 & 72.30 & 4.595 & 132.42 & 6.621 & 271.55 \\
\hline 0.127 & 4.02 & 2.592 & 73.04 & 4.641 & 135.22 & 6.647 & 273.75 \\
\hline 0.148 & 4.74 & 2.614 & 73.50 & 4.653 & 135.62 & 6.669 & 275.25 \\
\hline 0.171 & 5.58 & 2.637 & 74.08 & 4.698 & 137.52 & 6.690 & 275.75 \\
\hline 0.184 & 6.12 & 2.650 & 74.54 & 4.722 & 140.62 & 6.711 & 276.20 \\
\hline 0.220 & 7.22 & 2.683 & 74.94 & 4.744 & 143.02 & 6.742 & 276.95 \\
\hline 0.240 & 8.02 & 2.707 & 75.80 & 4.770 & 145.12 & 6.762 & 277.40 \\
\hline 0.247 & 8.34 & 2.729 & 76.44 & 4.788 & 145.82 & 6.784 & 277.70 \\
\hline 0.278 & 9.00 & 2.741 & 76.64 & 4.812 & 146.52 & 6.803 & 278.25 \\
\hline 0.290 & 9.34 & 2.754 & 77.12 & 4.821 & 146.92 & 6.880 & 280.75 \\
\hline 0.308 & 9.72 & 2.776 & 77.98 & 4.847 & 147.72 & 6.912 & 281.50 \\
\hline 0.326 & 10.10 & 2.798 & 78.32 & 4.866 & 148.22 & 6.933 & 282.00 \\
\hline 0.349 & 11.30 & 2.823 & 78.80 & 4.896 & 149.02 & 6.954 & 283.05 \\
\hline 0.388 & 12.46 & 2.853 & 79.58 & 4.919 & 149.92 & 6.976 & 284.65 \\
\hline 0.405 & 12.98 & 2.876 & 80.16 & 4.940 & 150.45 & 6.999 & 285.30 \\
\hline 0.422 & 13.22 & 2.904 & 80.78 & 4.962 & 151.40 & 7.026 & 285.84 \\
\hline 0.446 & 13.90 & 2.926 & 81.38 & 4.984 & 152.50 & 7.070 & 286.69 \\
\hline 0.463 & 14.34 & 2.937 & 81.84 & 5,011 & 154.80 & 7.117 & 288.64 \\
\hline 0.479 & 14.82 & 2.949 & 81.96 & 5.033 & 155.60 & 7.141 & 290.44 \\
\hline 0.526 & 16.48 & 2.969 & 82.50 & 5.055 & 156.80 & 7.163 & 291.24 \\
\hline 0.579 & 17.50 & 2.994 & 83.12 & 5.077 & 157.80 & 7.204 & 293.64 \\
\hline 0.636 & 18.76 & 3.019 & 83.68 & 5.101 & 158.60 & 7.280 & 295.14 \\
\hline 0.681 & 20.24 & 3.030 & 84.16 & 5.127 & 160.10 & 7.315 & 296.49 \\
\hline 0.737 & 21.92 & 3.041 & 84.46 & 5.148 & 160.40 & 7.357 & 298.34 \\
\hline 0.783 & 23.00 & 3.063 & 85.02 & 5.170 & 162.00 & 7.404 & 300.64 \\
\hline 0.805 & 23.24 & 3.085 & 85.58 & 5.190 & 162.80 & 7.449 & 302.24 \\
\hline 0.853 & 24.56 & 3.135 & 86.76 & 5.224 & 164.05 & 7.474 & 302.99 \\
\hline 0.874 & 25.28 & 3.156 & 87.42 & 5.264 & 166.60 & 7.496 & 303.44 \\
\hline 0.908 & 26.16 & 3.177 & 87.96 & 5.282 & 167.50 & 7.623 & 306.34 \\
\hline 0.936 & 26.86 & 3.198 & 88.66 & 5.300 & 168.45 & 7.774 & 306.39 \\
\hline 0.957 & 27.36 & 3.252 & 90.36 & 5.320 & 171.85 & 7.784 & 306.74 \\
\hline 0.978 & 27.62 & 3.271 & 90.88 & 5.342 & 174.00 & 7.807 & 307.89 \\
\hline 0.999 & 28.26 & 3.288 & 91.36 & 5.392 & 177.20 & 7.830 & 308.44 \\
\hline 1.050 & 29.44 & 3.298 & 91.72 & 5.414 & 179.45 & 7.878 & 309.94 \\
\hline 1.072 & 30.54 & 3.318 & 92.14 & 5.435 & 180.65 & 7.928 & 311.54 \\
\hline 1.092 & 30.92 & 3.348 & 92.92 & 5.457 & 181.35 & 7.946 & 312.29 \\
\hline 1.114 & 31.56 & 3.359 & 93.34 & 5.479 & 182.75 & 7.972 & 312.94 \\
\hline 1.136 & 32.06 & 3.370 & 93.62 & 5.507 & 183.55 & 7.993 & 313.39 \\
\hline 1.187 & 33.20 & 3.391 & 93.98 & 5.528 & 184.75 & 8.032 & 314.09 \\
\hline 1.215 & 33.76 & 3.401 & 94.52 & 5.549 & 185.70 & 8.144 & 316.14 \\
\hline 1.263 & 35.32 & 3.422 & 95.00 & 5.587 & 188.45 & 8.164 & 316.39 \\
\hline 1.283 & 36.20 & 3.443 & 95.46 & 5.603 & 190.25 & 8.186 & 317.09 \\
\hline 1.337 & 37.94 & 3.464 & 95.94 & 5.624 & 191.45 & 8.208 & 318.19 \\
\hline 1.369 & 39.50 & 3.496 & 97.12 & 5.643 & 193.00 & 8.235 & 318.99 \\
\hline 1.389 & 39.90 & 3.537 & 98.04 & 5.661 & 193.90 & 8.258 & 320.34 \\
\hline 1.400 & 40.08 & 3.567 & 98.76 & 5.680 & 195.45 & 8.279 & 320.89 \\
\hline 1.431 & 40.56 & 3.587 & 99.66 & 5.721 & 197.50 & 8.301 & 321.84 \\
\hline 1.463 & 41.28 & 3.595 & 99.96 & 5.752 & 199.70 & 8.322 & 322.79 \\
\hline 1.493 & 41.78 & 3.614 & 100.28 & 5.772 & 201.75 & 8.351 & 324.29 \\
\hline 1.528 & 42.72 & 3.633 & 100.78 & 5.794 & 204.40 & 8.434 & 325.64 \\
\hline 1.567 & 43.82 & 3.654 & 101.32 & 5.815 & 209.45 & 8.470 & 326.64 \\
\hline 1.586 & 44.30 & 3.689 & 102.53 & 5.836 & 211.10 & 8.489 & 326.93 \\
\hline 1.606 & 44.88 & 3.709 & 103.05 & 5.864 & 214.80 & 8.508 & 327.28 \\
\hline 1.645 & 45.62 & 3.730 & 103.73 & 5.887 & 215.75 & 8.547 & 327.93 \\
\hline 1.664 & 46.22 & 3.751 & 103.91 & 5.909 & 216.45 & 8.588 & 328.83 \\
\hline 1.697 & 46.68 & 3.781 & 104.81 & 5.929 & 216.75 & 8.600 & 329.43 \\
\hline 1.718 & 47.88 & 3.802 & 105.85 & 5.951 & 217.25 & 8.661 & 330.23 \\
\hline 1.739 & 48.28 & 3.824 & 106.23 & 5.981 & 218.05 & 8.682 & 330.68 \\
\hline 1.760 & 48.90 & 3.845 & 106.95 & 6.043 & 223.40 & 8.732 & 331.43 \\
\hline 1.782 & 49.24 & 3.867 & 107.51 & 6.060 & 225.95 & 8.796 & 332.73 \\
\hline 1.811 & 50.18 & 3.896 & 108.17 & 6.080 & 228.15 & 8.816 & 333.33 \\
\hline 1.832 & 50.66 & 3.918 & 108.75 & 6.099 & 229.45 & 8.926 & 335.13 \\
\hline 1.854 & 51.54 & 3.939 & 109.35 & 6.121 & 230.30 & 8.947 & 335.48 \\
\hline 1.916 & 53.92 & 3.960 & 109.87 & 6.138 & 231.05 & 8.968 & 335.83 \\
\hline 1.947 & 54.48 & 4.014 & 111.39 & 6.154 & 232.56 & 8.990 & 336.33 \\
\hline 1.958 & 55.38 & 4.034 & 111.83 & 6.174 & 234.31 & 9.062 & 337.88 \\
\hline 2.003 & 57.22 & 4.055 & 112.47 & 6.195 & 235.11 & 9.155 & 339.73 \\
\hline 2.023 & 57.94 & 4.072 & 112.85 & 6.215 & 237.16 & 9.199 & 340.53 \\
\hline 2.052 & 59.24 & 4.089 & 113.43 & 6.239 & 238.16 & 9.218 & 341.28 \\
\hline 2.097 & 60.88 & 4.110 & 113.99 & 6.267 & 240.06 & 9.397 & 344.13 \\
\hline 2.129 & 61.42 & 4.131 & 114.49 & 6.289 & 240.96 & 9.465 & 345.23 \\
\hline 2.140 & 61.82 & 4.154 & 115.21 & 6.309 & 241.61 & 9.511 & 346.08 \\
\hline 2.211 & 63.20 & 4.204 & 116.59 & 6.330 & 243.06 & 9.583 & 347.48 \\
\hline 2.233 & 63.68 & 4.215 & 116.99 & 6.349 & 243.86 & 9.682 & 348.63 \\
\hline 2.278 & 64.72 & 4.246 & 117.81 & 6.383 & 245.56 & 9.704 & 348.98 \\
\hline 2.305 & 65.58 & 4.272 & 118.67 & 6.404 & 246.21 & 9.815 & 350.08 \\
\hline 2.325 & 65.92 & 4.296 & 119.39 & 6.423 & 247.26 & 10.548 & 360.98 \\
\hline 2.348 & 66.56 & 4.319 & 120.03 & 6.443 & 248.51 & 10.693 & 364.33 \\
\hline 2.367 & 66.88 & 4.339 & 121.05 & 6.461 & 249.71 & 11.212 & 379.07 \\
\hline 2.388 & 67.46 & 4.412 & 123.25 & 6.480 & 250.91 & 11.420 & 384.72 \\
\hline 2.428 & 68.44 & 4.451 & 124.02 & 6.501 & 254.21 & 11.600 & 387.42 \\
\hline 2.462 & 69.68 & 4.491 & 125.42 & 6.535 & 259.21 & & \\
\hline
\end{tabular}


Table 7. Age model for Site 850 .

\begin{tabular}{|c|c|c|c|c|c|c|c|c|c|}
\hline $\begin{array}{l}\text { Age } \\
\text { (Ma) }\end{array}$ & $\begin{array}{l}\text { Depth } \\
\text { (mcd) }\end{array}$ & $\begin{array}{l}\text { Age } \\
(\mathrm{Ma})\end{array}$ & $\begin{array}{l}\text { Depth } \\
\text { (mcd) }\end{array}$ & $\begin{array}{l}\text { Age } \\
\text { (Ma) }\end{array}$ & $\begin{array}{l}\text { Depth } \\
\text { (mcd) }\end{array}$ & $\begin{array}{l}\text { Age } \\
\text { (Ma) }\end{array}$ & $\begin{array}{l}\text { Depth } \\
\text { (mcd) }\end{array}$ & $\begin{array}{l}\text { Age } \\
\text { (Ma) }\end{array}$ & $\begin{array}{l}\text { Depth } \\
\text { (mcd) }\end{array}$ \\
\hline 0.000 & 0.00 & 2.023 & 40.09 & 3.614 & 75.31 & 5.643 & 146.20 & 8.186 & 277.70 \\
\hline 0.039 & 0.75 & 2.052 & 40.73 & 3.633 & 75.71 & 5.661 & 146.80 & 8.188 & 277.80 \\
\hline 0.056 & 1.03 & 2.097 & 41.69 & 3.654 & 76.17 & 5.680 & 148.10 & 8.197 & 277.10 \\
\hline 0.088 & 1.65 & 2.118 & 41.97 & 3.689 & 76.99 & 5.700 & 148.90 & 8.258 & 279.60 \\
\hline 0.127 & 2.31 & 2.129 & 42.15 & 3.709 & 77.49 & 5.721 & 150.20 & 8.301 & 281.50 \\
\hline 0.148 & 3.21 & 2.140 & 42.45 & 3.730 & 78.17 & 5.752 & 152.00 & 8.322 & 282.60 \\
\hline 0.171 & 3.89 & 2.190 & 43.29 & 3.751 & 78.43 & 5.762 & 155.20 & 8.373 & 285.40 \\
\hline 0.220 & 4.77 & 2.201 & 43.45 & 3.781 & 79.05 & 5.794 & 158.00 & 8.434 & 287.90 \\
\hline 0.240 & 5.11 & 2.211 & 43.71 & 3.802 & 79.63 & 5.815 & 159.30 & 8.470 & 289.20 \\
\hline 0.247 & 5.45 & 2.222 & 43.93 & 3.824 & 80.15 & 5.836 & 160.50 & 8.489 & 289.80 \\
\hline 0.278 & 5.87 & 2.233 & 44.05 & 3.835 & 80.49 & 5.864 & 161.40 & 8.508 & 290.70 \\
\hline 0.290 & 5.99 & 2.255 & 44.65 & 3.855 & 80.71 & 5.893 & 163.10 & 8.525 & 291.20 \\
\hline 0.308 & 6.53 & 2.278 & 45.09 & 3.867 & 81.01 & 5.909 & 164.30 & 8.547 & 291.80 \\
\hline 0.326 & 6.69 & 2.305 & 45.75 & 3.896 & 81.59 & 5.929 & 165.10 & 8.567 & 292.30 \\
\hline 0.333 & 6.95 & 2.316 & 46.17 & 3.918 & 82.13 & 5.951 & 166.50 & 8.588 & 293.00 \\
\hline 0.349 & 7.43 & 2.325 & 46.45 & 3.939 & 82.61 & 5.981 & 168.00 & 8.661 & 296.00 \\
\hline 0.372 & 7.85 & 2.336 & 46.75 & 3.960 & 83.15 & 6.003 & 170.60 & 8.682 & 296.80 \\
\hline 0.388 & 8.33 & 2.377 & 48.27 & 4.034 & 84.47 & 6.022 & 172.20 & 8.685 & 296.90 \\
\hline 0.405 & 8.67 & 2.390 & 48.59 & 4.055 & 84.87 & 6.023 & 172.80 & 8.755 & 297.40 \\
\hline 0.422 & 8.91 & 2.411 & 49.41 & 4.072 & 85.15 & 6.059 & 175.50 & 8.776 & 297.80 \\
\hline 0.446 & 9.41 & 2.422 & 49.65 & 4.089 & 85.65 & 6.121 & 178.70 & 8.796 & 298.80 \\
\hline 0.463 & 9.71 & 2.438 & 49.89 & 4.110 & 86.03 & 6.174 & 181.80 & 8.833 & 300.30 \\
\hline 0.479 & 10.29 & 2.477 & 50.31 & 4.131 & 86.39 & 6.190 & 182.40 & 8.851 & 301.10 \\
\hline 0.504 & 10.51 & 2.490 & 50.63 & 4.154 & 86.79 & 6.195 & 182.50 & 8.872 & 302.10 \\
\hline 0.526 & 10.85 & 2.534 & 51.29 & 4.204 & 87.39 & 6.215 & 184.90 & 8.890 & 303.40 \\
\hline 0.579 & 11.59 & 2.558 & 51.81 & 4.225 & 87.41 & 6.267 & 188.50 & 8.926 & 304.90 \\
\hline 0.628 & 12.53 & 2.580 & 52.43 & 4.246 & 87.77 & 6.289 & 189.40 & 8.990 & 306.20 \\
\hline 0.659 & 13.69 & 2.604 & 52.91 & 4.272 & 88.45 & 6.309 & 190.40 & 9.002 & 306.70 \\
\hline 0.681 & 14.11 & 2.625 & 53.39 & 4.296 & 88.93 & 6.330 & 191.10 & 9.031 & 307.00 \\
\hline 0.692 & 14.31 & 2.650 & 53.93 & 4.319 & 89.32 & 6.336 & 191.30 & 9.107 & 310.30 \\
\hline 0.717 & 14.73 & 2.661 & 54.21 & 4.339 & 90.22 & 6.373 & 191.60 & 9.133 & 310.80 \\
\hline 0.737 & 15.09 & 2.683 & 54.49 & 4.361 & 91.32 & 6.383 & 192.10 & 9.155 & 311.40 \\
\hline 0.749 & 15.37 & 2.707 & 55.19 & 4.412 & 92.23 & 6.404 & 193.10 & 9.199 & 312.31 \\
\hline 0.772 & 15.63 & 2.729 & 55.47 & 4.451 & 93.15 & 6.501 & 198.50 & 9.221 & 312.91 \\
\hline 0.805 & 16.27 & 2.754 & 56.33 & 4.470 & 93.54 & 6.575 & 210.50 & 9.251 & 313.61 \\
\hline 0.824 & 16.61 & 2.798 & 57.33 & 4.490 & 94.46 & 6.647 & 220.91 & 9.269 & 314.11 \\
\hline 0.863 & 17.57 & 2.835 & 58.05 & 4.513 & 94.93 & 6.690 & 225.11 & 9.291 & 314.81 \\
\hline 0.884 & 17.99 & 2.853 & 58.55 & 4.537 & 96.06 & 6.711 & 226.01 & 9.305 & 315.41 \\
\hline 0.908 & 18.51 & 2.864 & 58.79 & 4.562 & 97.24 & 6.762 & 227.11 & 9.315 & 315.71 \\
\hline 0.978 & 19.65 & 2.876 & 58.95 & 4.583 & 97.82 & 6.803 & 228.11 & 9.381 & 316.71 \\
\hline 0.999 & 19.93 & 2.926 & 60.23 & 4.606 & 99.97 & 6.954 & 233.21 & 9.397 & 317.21 \\
\hline 1.029 & 20.53 & 2.937 & 60.59 & 4.629 & 101.09 & 6.999 & 235.11 & 9.420 & 318.01 \\
\hline 1.050 & 20.87 & 2.949 & 60.79 & 4.653 & 102.50 & 7.026 & 236.41 & 9.443 & 318.71 \\
\hline 1.072 & 21.81 & 2.969 & 61.25 & 4.698 & 106.10 & 7.070 & 237.50 & 9.465 & 319.51 \\
\hline 1.092 & 22.19 & 2.981 & 61.61 & 4.744 & 107.20 & 7.110 & 239.40 & 9.489 & 320.11 \\
\hline 1.136 & 23.17 & 2.994 & 61.73 & 4.770 & 108.90 & 7.120 & 239.90 & 9.511 & 321.01 \\
\hline 1.166 & 23.69 & 3.019 & 62.23 & 4.812 & 110.30 & 7.141 & 240.70 & 9.535 & 322.11 \\
\hline 1.187 & 23.97 & 3.041 & 62.81 & 4.866 & 112.20 & 7.163 & 242.00 & 9.626 & 324.51 \\
\hline 1.205 & 24.19 & 3.063 & 63.05 & 4.900 & 114.30 & 7.204 & 245.80 & 9.662 & 326.21 \\
\hline 1.224 & 24.57 & 3.085 & 63.53 & 5.011 & 115.30 & 7.238 & 246.70 & 9.677 & 326.41 \\
\hline 1.243 & 24.91 & 3.111 & 64.11 & 5.055 & 116.70 & 7.257 & 247.60 & 9.682 & 326.81 \\
\hline 1.263 & 25.07 & 3.135 & 64.65 & 5.101 & 118.30 & 7.280 & 248.80 & 9.735 & 328.01 \\
\hline 1.283 & 25.45 & 3.156 & 65.41 & 5.127 & 119.70 & 7.315 & 250.20 & 9.777 & 329.71 \\
\hline 1.317 & 26.29 & 3.177 & 66.01 & 5.159 & 120.60 & 7.380 & 253.00 & 9.798 & 330.21 \\
\hline 1.337 & 26.81 & 3.198 & 66.25 & 5.170 & 121.10 & 7.404 & 253.90 & 9.869 & 332.21 \\
\hline 1.358 & 27.31 & 3.214 & 66.53 & 5.224 & 122.60 & 7.428 & 255.20 & 9.963 & 334.91 \\
\hline 1.379 & 27.55 & 3.231 & 66.79 & 5.264 & 124.40 & 7.449 & 256.20 & 9.985 & 335.81 \\
\hline 1.400 & 27.97 & 3.252 & 67.23 & 5.270 & 124.90 & 7.474 & 257.30 & 10.070 & 336.01 \\
\hline 1.463 & 29.25 & 3.262 & 67.45 & 5.282 & 125.30 & 7.496 & 258.00 & 10.270 & 345.51 \\
\hline 1.473 & 29.37 & 3.271 & 67.67 & 5.300 & 126.70 & 7.525 & 258.90 & 10.370 & 345.71 \\
\hline 1.493 & 29.51 & 3.288 & 68.01 & 5.310 & 128.80 & 7.645 & 259.00 & 10.400 & 347.01 \\
\hline 1.504 & 29.89 & 3.328 & 68.85 & 5.320 & 129.10 & 7.650 & 259.20 & 10.490 & 350.61 \\
\hline 1.528 & 30.37 & 3.338 & 69.01 & 5.342 & 131.50 & 7.740 & 262.40 & 10.510 & 351.41 \\
\hline 1.547 & 30.85 & 3.348 & 69.15 & 5.363 & 133.80 & 7.763 & 263.50 & 10.520 & 355.31 \\
\hline 1.567 & 31.17 & 3.359 & 69.41 & 5.370 & 134.20 & 7.808 & 265.60 & 10.693 & 363.51 \\
\hline 1.606 & 31.91 & 3.391 & 69.91 & 5.410 & 134.70 & 7.830 & 266.30 & 10.725 & 364.71 \\
\hline 1.697 & 33.29 & 3.401 & 70.13 & 5.414 & 134.80 & 7.855 & 266.90 & 10.757 & 364.91 \\
\hline 1.739 & 34.55 & 3.422 & 70.61 & 5.435 & 136.30 & 7.878 & 268.10 & 10.843 & 368.11 \\
\hline 1.750 & 34.73 & 3.433 & 71.03 & 5.457 & 137.40 & 7.955 & 268.20 & 11.154 & 382.71 \\
\hline 1.811 & 35.91 & 3.443 & 71.15 & 5.479 & 138.70 & 7.955 & 268.50 & 11.413 & 393.51 \\
\hline 1.832 & 36.35 & 3.464 & 71.61 & 5.507 & 139.80 & 7.972 & 269.10 & 11.547 & 393.91 \\
\hline 1.904 & 37.73 & 3.496 & 72.49 & 5.549 & 141.10 & 7.993 & 270.40 & 11.950 & 407.31 \\
\hline 1.916 & 38.19 & 3.516 & 72.87 & 5.569 & 142.30 & 8.032 & 271.60 & & \\
\hline 1.958 & 38.93 & 3.537 & 73.29 & 5.587 & 143.90 & 8.069 & 273.10 & & \\
\hline 1.986 & 39.31 & 3.567 & 74.19 & 5.614 & 144.20 & 8.090 & 273.90 & & \\
\hline 2.003 & 39.69 & 3.595 & 74.89 & 5.624 & 144.70 & 8.144 & 275.60 & & \\
\hline
\end{tabular}

Age control points then were added so as to align prominent groups of density maxima with groups of insolation peaks. We found that sections having about $0.8-\mathrm{m}$.y. duration were conveniently viewed. Each of the sites containing orbital scale variability over a chosen time interval was first tuned in this fashion independently. Next, records were compared with each other and, if necessary, with other lower-resolution sites containing magnetostratigraphic data. Because aboard the ship we had observed close similarities among the GRAPE density records of sites even when widely separated, we have assumed throughout this exercise that changes in percentage carbonate (as reflected in the GRAPE density records) in reality did occur synchronously over wide areas of the Pacific Basin. Many previous studies have been based successfully on this hypothesis (Arrhenius, 1952; Hays et al., 1969; Vincent, 1981; Farrell and Prell, 1991). 
Table 8. Age model for Site 851.

\begin{tabular}{|c|c|c|c|c|c|c|c|c|c|}
\hline $\begin{array}{l}\text { Age } \\
\text { (Ma) }\end{array}$ & $\begin{array}{l}\text { Depth } \\
\text { (mcd) }\end{array}$ & $\begin{array}{l}\text { Age } \\
\text { (Ma) }\end{array}$ & $\begin{array}{l}\text { Depth } \\
\text { (mcd) }\end{array}$ & $\begin{array}{l}\text { Age } \\
\text { (Ma) }\end{array}$ & $\begin{array}{l}\text { Depth } \\
\text { (mcd) }\end{array}$ & $\begin{array}{l}\text { Age } \\
(\mathrm{Ma})\end{array}$ & $\begin{array}{l}\text { Depth } \\
\text { (mcd) }\end{array}$ & $\begin{array}{l}\text { Age } \\
\text { (Ma) }\end{array}$ & $\begin{array}{l}\text { Depth } \\
\text { (mcd) }\end{array}$ \\
\hline 0.000 & 0.00 & 1.916 & 35.48 & 3.391 & 63.80 & 5.435 & 111.29 & 7.541 & 209.85 \\
\hline 0.039 & 0.82 & 1.947 & 35.80 & 3.401 & 64.20 & 5.457 & 111.81 & 7.565 & 210.40 \\
\hline 0.065 & 1.44 & 1.958 & 36.14 & 3.422 & 64.56 & 5.479 & 112.98 & 7.592 & 211.85 \\
\hline 0.088 & 1.84 & 2.003 & 36.80 & 3.443 & 65.16 & 5.507 & 114.37 & 7.611 & 212.65 \\
\hline 0.127 & 2.48 & 2.023 & 37.28 & 3.464 & 65.36 & 5.528 & 115.07 & 7.650 & 214.35 \\
\hline 0.148 & 2.78 & 2.041 & 37.84 & 3.475 & 65.76 & 5.549 & 116.16 & 7.691 & 216.60 \\
\hline 0.171 & 2.98 & 2.052 & 38.06 & 3.496 & 66.08 & 5.587 & 118.36 & 7.740 & 218.30 \\
\hline 0.184 & 3.16 & 2.097 & 38.74 & 3.516 & 66.48 & 5.603 & 119.03 & 7.763 & 219.35 \\
\hline 0.220 & 3.84 & 2.118 & 39.00 & 3.537 & 66.78 & 5.624 & 119.88 & 7.784 & 220.50 \\
\hline 0.240 & 4.00 & 2.129 & 39.12 & 3.567 & 67.32 & 5.643 & 120.45 & 7.807 & 221.50 \\
\hline 0.247 & 4.26 & 2.140 & 39.54 & 3.604 & 67.94 & 5.661 & 121.38 & 7.830 & 222.15 \\
\hline 0.278 & 4.66 & 2.190 & 40.24 & 3.623 & 68.56 & 5.680 & 122.42 & 7.855 & 223.10 \\
\hline 0.308 & 4.88 & 2.211 & 40.58 & 3.654 & 69.08 & 5.700 & 122.88 & 7.878 & 223.95 \\
\hline 0.326 & 5.22 & 2.233 & 40.94 & 3.689 & 69.72 & 5.721 & 123.73 & 7.899 & 225.55 \\
\hline 0.349 & 5.72 & 2.278 & 42.04 & 3.720 & 70.26 & 5.752 & 124.64 & 7.921 & 226.00 \\
\hline 0.388 & 6.52 & 2.293 & 42.22 & 3.764 & 70.84 & 5.772 & 125.99 & 7.972 & 228.30 \\
\hline 0.405 & 6.70 & 2.305 & 42.36 & 3.781 & 71.02 & 5.794 & 127.88 & 7.993 & 229.50 \\
\hline 0.422 & 6.90 & 2.316 & 42.48 & 3.792 & 71.30 & 5.815 & 129.07 & 8.069 & 231.46 \\
\hline 0.446 & 7.50 & 2.325 & 42.68 & 3.813 & 71.50 & 5.836 & 130.91 & 8.144 & 233.81 \\
\hline 0.479 & 8.26 & 2.336 & 42.96 & 3.824 & 71.64 & 5.864 & 131.85 & 8.186 & 234.86 \\
\hline 0.479 & 9.66 & 2.377 & 44.74 & 3.835 & 71.90 & 5.887 & 132.47 & 8.208 & 235.36 \\
\hline 0.510 & 10.28 & 2.390 & 45.12 & 3.845 & 72.08 & 5.909 & 133.27 & 8.235 & 235.91 \\
\hline 0.523 & 10.54 & 2.411 & 45.58 & 3.867 & 72.26 & 5.929 & 133.80 & 8.247 & 236.16 \\
\hline 0.544 & 10.82 & 2.438 & 46.00 & 3.884 & 72.58 & 5.951 & 134.38 & 8.301 & 236.26 \\
\hline 0.579 & 11.30 & 2.465 & 46.42 & 3.896 & 72.74 & 5.966 & 134.87 & 8.351 & 238.46 \\
\hline 0.614 & 11.62 & 2.477 & 46.60 & 3.918 & 73.04 & 5.981 & 135.28 & 8.373 & 239.26 \\
\hline 0.628 & 11.80 & 2.490 & 46.92 & 3.939 & 73.38 & 6.003 & 135.78 & 8.394 & 239.91 \\
\hline 0.636 & 12.00 & 2.534 & 47.56 & 3.960 & 73.70 & 6.043 & 136.89 & 8.434 & 242.11 \\
\hline 0.659 & 12.66 & 2.547 & 47.80 & 4.004 & 74.38 & 6.060 & 137.69 & 8.470 & 243.91 \\
\hline 0.681 & 13.18 & 2.569 & 48.02 & 4.034 & 74.72 & 6.121 & 139.84 & 8.508 & 244.51 \\
\hline 0.717 & 14.24 & 2.592 & 48.52 & 4.110 & 75.80 & 6.215 & 146.86 & 8.547 & 245.96 \\
\hline 0.737 & 14.54 & 2.614 & 49.00 & 4.154 & 77.08 & 6.267 & 149.76 & 8.661 & 249.11 \\
\hline 0.783 & 15.30 & 2.637 & 49.26 & 4.204 & 77.50 & 6.289 & 150.67 & 8.682 & 249.46 \\
\hline 0.805 & 15.70 & 2.650 & 49.60 & 4.225 & 77.96 & 6.309 & 151.28 & 8.704 & 249.81 \\
\hline 0.824 & 15.90 & 2.661 & 49.84 & 4.246 & 78.44 & 6.383 & 153.76 & 8.755 & 251.26 \\
\hline 0.863 & 16.60 & 2.684 & 50.04 & 4.272 & 79.08 & 6.404 & 154.41 & 8.776 & 252.61 \\
\hline 0.884 & 16.92 & 2.707 & 50.36 & 4.319 & 79.92 & 6.423 & 155.18 & 8.816 & 254.41 \\
\hline 0.908 & 17.60 & 2.718 & 50.62 & 4.339 & 80.56 & 6.461 & 157.49 & 8.833 & 255.31 \\
\hline 0.925 & 17.90 & 2.729 & 51.00 & 4.361 & 81.36 & 6.501 & 158.94 & 8.872 & 258.01 \\
\hline 0.978 & 18.80 & 2.754 & 51.82 & 4.412 & 82.28 & 6.518 & 160.55 & 8.890 & 258.76 \\
\hline 0.999 & 19.12 & 2.766 & 52.24 & 4.451 & 83.04 & 6.535 & 163.85 & 8.907 & 259.41 \\
\hline 1.050 & 19.80 & 2.776 & 52.44 & 4.491 & 84.08 & 6.554 & 165.30 & 8.926 & 260.06 \\
\hline 1.072 & 20.30 & 2.798 & 52.72 & 4.513 & 84.64 & 6.575 & 167.00 & 8.947 & 260.81 \\
\hline 1.092 & 20.98 & 2.835 & 53.36 & 4.537 & 84.96 & 6.596 & 168.20 & 8.990 & 262.30 \\
\hline 1.114 & 21.54 & 2.853 & 53.80 & 4.562 & 85.62 & 6.621 & 174.65 & 9.018 & 263.20 \\
\hline 1.136 & 21.88 & 2.904 & 54.68 & 4.583 & 85.84 & 6.640 & 177.20 & 9.062 & 265.25 \\
\hline 1.166 & 22.40 & 2.926 & 55.24 & 4.595 & 86.42 & 6.669 & 178.85 & 9.084 & 265.85 \\
\hline 1.205 & 23.10 & 2.937 & 55.68 & 4.641 & 87.72 & 6.690 & 179.65 & 9.199 & 269.50 \\
\hline 1.215 & 23.32 & 2.949 & 55.84 & 4.665 & 88.74 & 6.711 & 180.75 & 9.269 & 271.50 \\
\hline 1.243 & 23.56 & 2.969 & 56.14 & 4.698 & 89.63 & 6.742 & 181.45 & 9.280 & 271.80 \\
\hline 1.263 & 24.14 & 2.994 & 56.50 & 4.722 & 90.18 & 6.803 & 183.40 & 9.348 & 272.00 \\
\hline 1.283 & 24.60 & 3.019 & 56.98 & 4.744 & 90.68 & 6.822 & 183.95 & 9.397 & 273.35 \\
\hline 1.317 & 25.18 & 3.030 & 57.44 & 4.770 & 91.64 & 6.839 & 184.85 & 9.420 & 274.00 \\
\hline 1.337 & 25.60 & 3.041 & 57.58 & 4.812 & 92.49 & 6.880 & 186.20 & 9.443 & 274.55 \\
\hline 1.379 & 26.32 & 3.063 & 57.94 & 4.835 & 93.12 & 6.954 & 188.25 & 9.465 & 275.20 \\
\hline 1.400 & 26.94 & 3.085 & 58.42 & 4.866 & 93.93 & 6.976 & 189.70 & 9.489 & 275.75 \\
\hline 1.431 & 27.46 & 3.095 & 58.48 & 4.878 & 94.59 & 6.999 & 190.25 & 9.511 & 276.50 \\
\hline 1.463 & 27.96 & 3.111 & 58.64 & 4.919 & 95.41 & 7.070 & 193.50 & 9.626 & 279.70 \\
\hline 1.493 & 28.78 & 3.135 & 59.08 & 4.940 & 96.00 & 7.117 & 195.45 & 9.682 & 282.50 \\
\hline 1.567 & 29.82 & 3.156 & 59.58 & 4.962 & 96.84 & 7.141 & 196.70 & 9.735 & 284.30 \\
\hline 1.595 & 30.22 & 3.177 & 59.90 & 4.997 & 98.08 & 7.163 & 197.55 & 9.798 & 286.25 \\
\hline 1.606 & 30.42 & 3.198 & 60.28 & 5.043 & 99.38 & 7.204 & 199.05 & 9.847 & 287.65 \\
\hline 1.645 & 30.96 & 3.231 & 60.80 & 5.087 & 100.94 & 7.238 & 199.90 & 9.892 & 289.10 \\
\hline 1.664 & 31.32 & 3.252 & 61.20 & 5.115 & 101.86 & 7.257 & 200.35 & 9.913 & 289.70 \\
\hline 1.697 & 31.64 & 3.271 & 61.66 & 5.159 & 102.81 & 7.280 & 200.90 & 9.939 & 290.35 \\
\hline 1.718 & 31.80 & 3.288 & 61.86 & 5.170 & 103.21 & 7.333 & 202.75 & 9.963 & 291.40 \\
\hline 1.750 & 32.84 & 3.308 & 62.38 & 5.190 & 103.56 & 7.380 & 203.95 & 10.022 & 293.05 \\
\hline 1.811 & 33.70 & 3.318 & 62.62 & 5.224 & 104.13 & 7.404 & 204.90 & 10.693 & 323.54 \\
\hline 1.832 & 34.00 & 3.328 & 62.90 & 5.320 & 107.41 & 7.449 & 205.75 & 11.212 & 346.08 \\
\hline 1.854 & 34.34 & 3.348 & 63.12 & 5.342 & 107.93 & 7.474 & 206.90 & 11.420 & 353.48 \\
\hline 1.875 & 34.70 & 3.359 & 63.32 & 5.392 & 109.09 & 7.496 & 207.45 & 12.000 & 370.63 \\
\hline 1.904 & 35.00 & 3.370 & 63.50 & 5.414 & 110.67 & 7.519 & 208.85 & & \\
\hline
\end{tabular}

Mayer (1991) showed how one may calculate a record of percentage carbonate from a GRAPE density record, and Hagelberg et al. (this volume) show carbonate records derived by this method for the Leg 138 sites. We chose to work with untransformed GRAPE density data because analytical uncertainty (and, hence, any measure of significance) is uniform across the density range, whereas it is not uniform across the percentage range. For logistical reasons, we initially worked with the spliced records as displayed in Hagelberg et al. (1992; Fig. 5); when a stacked record for a site (Hagelberg et al., this volume) became available, this was used instead. Consequently, not all the records were worked on at the same degree of smoothing. In general, working with the stacked and smoothed records was easier. For the plots that are shown in this chapter as Figures 1A through $1 \mathrm{~F}$, all the GRAPE density data were smoothed in the time domain.

\section{RESULTS}

In this section, we discuss the results of tuning the Leg 138 sites in 1-m.y. sections, starting from the most recent increment. The outcome is reported in the series of Tables 1 through 11 that lists depth- 
Table 9. Age model for Site 852 .

\begin{tabular}{|c|c|c|c|c|c|}
\hline $\begin{array}{l}\text { Age } \\
\text { (Ma) }\end{array}$ & $\begin{array}{l}\text { Depth } \\
\text { (mcd) }\end{array}$ & $\begin{array}{l}\text { Age } \\
\text { (Ma) }\end{array}$ & $\begin{array}{l}\text { Depth } \\
\text { (mcd) }\end{array}$ & $\begin{array}{l}\text { Age } \\
\text { (Ma) }\end{array}$ & $\begin{array}{l}\text { Depth } \\
\text { (mcd) }\end{array}$ \\
\hline 0.000 & 0.00 & 2.637 & 30.15 & 4.919 & 56.37 \\
\hline 0.039 & 0.53 & 2.683 & 30.57 & 4.940 & 56.71 \\
\hline 0.056 & 0.73 & 2.718 & 30.95 & 4.984 & 57.47 \\
\hline 0.088 & 1.11 & 2.729 & 31.15 & 4.997 & 57.61 \\
\hline 0.127 & 1.65 & 2.766 & 31.95 & 5.055 & 58.57 \\
\hline 0.171 & 2.17 & 2.798 & 32.25 & 5.087 & 59.15 \\
\hline 0.184 & 2.23 & 2.835 & 32.61 & 5.115 & 59.75 \\
\hline 0.220 & 2.37 & 2.853 & 33.03 & 5.159 & 60.81 \\
\hline 0.240 & 2.57 & 2.864 & 33.15 & 5.200 & 61.25 \\
\hline 0.247 & 2.65 & 2.876 & 33.25 & 5.224 & 61.55 \\
\hline 0.278 & 2.93 & 2.892 & 33.41 & 5.264 & 62.15 \\
\hline 0.308 & 3.11 & 2.904 & 33.63 & 5.320 & 63.21 \\
\hline 0.326 & 3.23 & 2.915 & 33.81 & 5.342 & 63.77 \\
\hline 0.349 & 3.61 & 2.926 & 33.91 & 5.363 & 64.11 \\
\hline 0.388 & 4.05 & 2.949 & 34.35 & 5.392 & 64.33 \\
\hline 0.422 & 4.37 & 2.969 & 34.45 & 5.435 & 65.13 \\
\hline 0.446 & 4.61 & 2.994 & 34.73 & 5.457 & 65.39 \\
\hline 0.479 & 5.11 & 3.019 & 34.85 & 5.479 & 65.81 \\
\hline 0.510 & 5.65 & 3.041 & 35.29 & 5.507 & 66.17 \\
\hline 0.579 & 6.31 & 3.063 & 35.47 & 5.528 & 66.47 \\
\hline 0.614 & 6.49 & 3.111 & 36.09 & 5.549 & 66.93 \\
\hline 0.628 & 6.77 & 3.131 & 36.25 & 5.587 & 67.37 \\
\hline 0.659 & 6.95 & 3.146 & 36.51 & 5.643 & 68.11 \\
\hline 0.681 & 7.51 & 3.198 & 37.11 & 5.680 & 68.59 \\
\hline 0.712 & 8.09 & 3.231 & 37.35 & 5.721 & 69.35 \\
\hline 0.717 & 8.17 & 3.252 & 37.53 & 5.732 & 69.53 \\
\hline 0.772 & 8.85 & 3.262 & 37.59 & 5.752 & 70.15 \\
\hline 0.783 & 9.05 & 3.271 & 37.77 & 5.783 & 70.49 \\
\hline 0.824 & 9.49 & 3.318 & 38.23 & 5.825 & 71.07 \\
\hline 0.863 & 10.09 & 3.333 & 38.39 & 5.864 & 71.69 \\
\hline 0.908 & 10.65 & 3.338 & 38.45 & 5.909 & 72.41 \\
\hline 0.957 & 11.17 & 3.348 & 38.57 & 5.951 & 72.97 \\
\hline 0.978 & 11.29 & 3.370 & 38.79 & 5.981 & 73.59 \\
\hline 1.050 & 12.17 & 3.391 & 38.91 & 6.003 & 74.07 \\
\hline 1.092 & 12.97 & 3.401 & 39.11 & 6.060 & 75.37 \\
\hline 1.114 & 13.25 & 3.411 & 39.25 & 6.080 & 75.81 \\
\hline 1,166 & 13.97 & 3.422 & 39.39 & 6.122 & 76.27 \\
\hline 1.205 & 14.25 & 3.443 & 39.69 & 6.195 & 77.91 \\
\hline 1.243 & 14.75 & 3.475 & 40.05 & 6.215 & 78.23 \\
\hline 1.283 & 15.35 & 3.496 & 40.21 & 6.239 & 78.67 \\
\hline 1.337 & 15.91 & 3.516 & 40.35 & 6.289 & 79.47 \\
\hline 1.379 & 16.57 & 3.537 & 40.51 & 6.383 & 80.53 \\
\hline 1.400 & 16.87 & 3.548 & 40.71 & 6.404 & 80.85 \\
\hline 1.431 & 17.21 & 3.567 & 40.83 & 6.480 & 81.67 \\
\hline 1.493 & 17.87 & 3.614 & 41.23 & 6.501 & 81.97 \\
\hline 1.528 & 18.19 & 3.680 & 41.65 & 6.621 & 83.91 \\
\hline 1.567 & 18.61 & 3.751 & 42.09 & 6.669 & 84.41 \\
\hline 1.606 & 19.17 & 3.781 & 42.55 & 6.742 & 85.21 \\
\hline 1.645 & 19.59 & 3.802 & 42.79 & 6.933 & 89.83 \\
\hline 1.664 & 19.79 & 3.824 & 42.95 & 7.404 & 95.53 \\
\hline 1.750 & 20.73 & 3.884 & 43.65 & 7.474 & 96.05 \\
\hline 1.811 & 21.39 & 3.918 & 43.93 & 7.519 & 96.75 \\
\hline 1.832 & 21.57 & 3.960 & 44.53 & 7.740 & 99.49 \\
\hline 1.854 & 21.71 & 4.034 & 45.15 & 7.763 & 99.95 \\
\hline 1.875 & 21.91 & 4.141 & 46.07 & 7.807 & 100.85 \\
\hline 1.947 & 22.51 & 4.204 & 46.51 & 7.855 & 101.71 \\
\hline 1.958 & 22.65 & 4.225 & 46.75 & 7.899 & 102.59 \\
\hline 1.986 & 22.93 & 4.246 & 46.97 & 7.921 & 103.17 \\
\hline 2.023 & 23,35 & 4.319 & 47.77 & 7.946 & 103.51 \\
\hline 2.042 & 23.57 & 4.339 & 48.31 & 8.027 & 105.02 \\
\hline 2,075 & 24.09 & 4.412 & 49.21 & 8.144 & 107.66 \\
\hline 2.097 & 24.45 & 4.451 & 49.81 & 8.164 & 107.96 \\
\hline 2.140 & 24.81 & 4.470 & 50.03 & 8.174 & 108.32 \\
\hline 2.190 & 25.33 & 4.491 & 50.33 & 8.635 & 114.36 \\
\hline 2.233 & 25.69 & 4.537 & 50.77 & 8.945 & 116.60 \\
\hline 2.278 & 25.99 & 4.583 & 51.31 & 9.142 & 117.30 \\
\hline 2.305 & 26.43 & 4.606 & 51.79 & 9.218 & 118.10 \\
\hline 2.336 & 26.73 & 4.629 & 51.87 & 9.482 & 119.04 \\
\hline 2.377 & 27.25 & 4.676 & 52.55 & 9.543 & 119.40 \\
\hline 2.390 & 27.43 & 4.698 & 53.11 & 9.639 & 119.64 \\
\hline 2.438 & 27.85 & 4.744 & 53.67 & 9.775 & 120.34 \\
\hline 2.477 & 28.37 & 4.758 & 54.11 & 9.815 & 120.50 \\
\hline 2.534 & 29.17 & 4.770 & 54.33 & 10.022 & 122.40 \\
\hline 2.558 & 29.37 & 4.812 & 54.87 & 10.548 & 127.60 \\
\hline 2.592 & 29.67 & 4.835 & 55.17 & 10.576 & 127.86 \\
\hline 2.604 & 29.75 & 4.878 & 55.87 & 10.830 & 128.30 \\
\hline
\end{tabular}

age pairs for recognizable GRAPE events at each site. Figures $1 \mathrm{~A}$ to 1F show GRAPE density for Sites 846 to 854 vs. age for each age interval; each panel covers 2 m.y. For each site, vertical lines show the positions of all age control points. To aid in comparison, all the GRAPE density data for Figure 1 were interpolated at 1-k.y. intervals and smoothed in the time domain using a Gaussian filter having a total width of 5.9 k.y. Where the reconstructed sedimentation rate falls
Table 10. Age model for Site 853.

\begin{tabular}{|c|c|c|c|}
\hline $\begin{array}{l}\text { Age } \\
\text { (Ma) }\end{array}$ & $\begin{array}{l}\text { Depth } \\
\text { (mcd) }\end{array}$ & $\begin{array}{l}\text { Age } \\
\text { (Ma) }\end{array}$ & $\begin{array}{l}\text { Depth } \\
\text { (mcd) }\end{array}$ \\
\hline 0.000 & 0.22 & 4.885 & 28.15 \\
\hline 0.473 & 1.77 & 5.004 & 28.96 \\
\hline 0.681 & 2.47 & 5.240 & 31.50 \\
\hline 0.780 & 2.95 & 5.875 & 38.57 \\
\hline 0.990 & 3.85 & 6.256 & 43.55 \\
\hline 1.070 & 4.19 & 6.554 & 46.52 \\
\hline 1.770 & 7.80 & 7.072 & 54.16 \\
\hline 1.950 & 8.57 & 7.318 & 55.56 \\
\hline 2.600 & 12.18 & 7.351 & 56.31 \\
\hline 3.054 & 15.23 & 7.406 & 56.55 \\
\hline 3.127 & 15.74 & 7.533 & 58.30 \\
\hline 3.221 & 16.33 & 7.618 & 59.04 \\
\hline 3.325 & 16.89 & 8.027 & 65.60 \\
\hline 3.612 & 18.82 & 8.173 & 68.40 \\
\hline 4.188 & 22.30 & 8.205 & 69.00 \\
\hline 4.320 & 23.53 & 8.635 & 75.06 \\
\hline 4.452 & 25.24 & 8.645 & 75.20 \\
\hline $\begin{array}{l}4.621 \\
4.801\end{array}$ & $\begin{array}{l}26.08 \\
27.41\end{array}$ & & \\
\hline
\end{tabular}

Table 11. Age model for Site 854.

\begin{tabular}{|c|c|c|c|}
\hline $\begin{array}{l}\text { Age } \\
\text { (Ma) }\end{array}$ & $\begin{array}{l}\text { Depth } \\
\text { (mcd) }\end{array}$ & $\begin{array}{l}\text { Age } \\
\text { (Ma) }\end{array}$ & $\begin{array}{l}\text { Depth } \\
\text { (mcd) }\end{array}$ \\
\hline 0.000 & 0.00 & 6.919 & 25.27 \\
\hline 0.780 & 4.08 & 7.072 & 27.57 \\
\hline 0.990 & 5.35 & 7.406 & 30.72 \\
\hline 1.070 & 5.82 & 7.533 & 32.69 \\
\hline 1.770 & 10.61 & 7.618 & 33.23 \\
\hline 1.950 & 11.48 & 8.027 & 39.78 \\
\hline 2.600 & 16.68 & 8.174 & 41.75 \\
\hline 3.053 & 17.68 & 8.205 & 42.30 \\
\hline 3.131 & 17.82 & 8.631 & 43.78 \\
\hline 3.224 & 17.95 & 8.945 & 45.40 \\
\hline 3.337 & 18.15 & 9.142 & 46.55 \\
\hline 3.611 & 19.08 & 9.218 & 47.33 \\
\hline 3.740 & 19.50 & 9.482 & 48.85 \\
\hline 5.875 & 19.93 & 9.543 & 49.48 \\
\hline 6.122 & 21.11 & 9.639 & 49.80 \\
\hline 6.256 & 21.91 & & \\
\hline 6.555 & 23.03 & & \\
\hline
\end{tabular}

below about $5 \mathrm{~m} / \mathrm{m} . \mathrm{y}$., as it does at several points in Site 848, the data are further smoothed; these intervals are not useful for tuning.

We show the results of tuning sections of individual holes from Sites 850,851 , and 852 in Figures 2 to 8 for two reasons. First, it is only by referring to the individual holes that one can assess the exact relationship between the GRAPE density and paleomagnetic stratigraphies. Second, these figures display the strengths and limitations of our tuning approach more clearly than Figure 1 does.

The interval from zero to $2 \mathrm{Ma}$ (Fig. 1A) was surprisingly difficult to tune, considering the amount of work that has been devoted to the study of Pleistocene climate. Examination of Figure 1A shows a convincing degree of correlation among the sites, but the relationship with the orbital data is not at all obvious. Here, we do not quantify the correlation among sites, but note that Hagelberg et al. (this volume) demonstrate by empirical orthogonal function (EOF) analysis that a high proportion of the variability in all the sites is explained by the first EOF.

Figure 2 shows the data for the interval 0 to $1 \mathrm{Ma}$ from Holes 851B, 851C, 851D, and 851E separately, tuned with GRAPE density extremes correlated to insolation extremes. In this interval, the match between GRAPE density and the orbital record is fairly poor. As regards tuning to the orbital record is concerned, we emphasize again that in this interval we have been guided by the objective of creating a time scale based on features in the GRAPE density record that is not grossly inconsistent with a $\delta^{18} \mathrm{O}$-based time scale. By contrast, in Figure 1, the GRAPE density data for Site 851 are shown using the control points in Table 8 , so that for the past million years the GRAPE density extremes are no longer all exactly aligned with insolation 
Table 12. Age-depth control points for the interval 0 to 1 Ma derived by correlating GRAPE density and orbitally controlled insolation without regard to the established $\delta^{18} \mathrm{O}$ time scale.

\begin{tabular}{|c|c|c|c|c|c|}
\hline $\begin{array}{l}\text { Age } \\
(\mathrm{Ma})\end{array}$ & $\begin{array}{l}\text { Depth } \\
\text { (mcd) }\end{array}$ & $\begin{array}{l}\text { Age } \\
\text { (Ma) }\end{array}$ & $\begin{array}{l}\text { Depth } \\
\text { (mcd) }\end{array}$ & $\begin{array}{l}\text { Age } \\
\text { (Ma) }\end{array}$ & $\begin{array}{l}\text { Depth } \\
\text { (mcd) }\end{array}$ \\
\hline Site 844 & & 0.218 & 2.80 & 0.631 & 12.79 \\
\hline 0.000 & 0.00 & 0.241 & 3.12 & 0.681 & 14.11 \\
\hline 0.780 & 10.04 & 0.262 & 3.44 & 0.692 & 14.31 \\
\hline 0.990 & 11.83 & 0.311 & 4.10 & 0.712 & 14.81 \\
\hline 1.070 & 12.36 & 0.354 & 4.70 & 0.749 & 15.09 \\
\hline & & 0.408 & 5.64 & 0.805 & 16.27 \\
\hline Site 845 & & 0.462 & 6.52 & 0.824 & 16.61 \\
\hline 0.000 & $\begin{array}{r}0.00 \\
50.00\end{array}$ & 0.484 & 7.42 & 0.863 & 17.57 \\
\hline 3.610 & 59.88 & 0.528 & 7.88 & 0.884 & 17.99 \\
\hline Site 846 & & 0.565 & 8.68 & 0.908 & 18.51 \\
\hline 0.000 & 0.00 & 0.609 & 9.30 & 0.978 & 19.65 \\
\hline 0.033 & 1.82 & 0.648 & 10.08 & 0.999 & 19.93 \\
\hline 0.056 & 3.04 & 0.681 & 10.80 & 1.029 & 20.53 \\
\hline 0.126 & 5.98 & 0.710 & 11.08 & & \\
\hline 0.148 & 6.76 & $\begin{array}{l}0.749 \\
0.787\end{array}$ & 11.86 & 0.000 & 0.00 \\
\hline 0.173 & 7.38 & $\begin{array}{l}0.787 \\
0.863\end{array}$ & $\begin{array}{l}12.70 \\
13.80\end{array}$ & 0.056 & 1.16 \\
\hline 0.218 & 8.52 & $\begin{array}{l}0.863 \\
0.908\end{array}$ & $\begin{array}{l}13.80 \\
14.86\end{array}$ & 0.082 & 1.62 \\
\hline 0.241 & 9.06 & 0.925 & 15.16 & 0.103 & 1.98 \\
\hline 0.262 & 9.94 & 0.978 & 15.76 & 0.126 & 2.48 \\
\hline 0.290 & 11.84 & 0.999 & 16.02 & 0.148 & 2.78 \\
\hline 0.311 & 12.06 & & & 0.173 & 2.98 \\
\hline 0.333 & 12.66 & Site 849 & & 0.218 & 3.76 \\
\hline 0.354 & 13.44 & 0.000 & 0.02 & 0.262 & 4.26 \\
\hline 0.386 & 14.70 & 0.056 & 1.58 & 0.290 & 4.88 \\
\hline 0.408 & 15.98 & 0.070 & 2.76 & 0.333 & 5.36 \\
\hline 0.462 & 17.72 & 0.082 & 3.18 & 0.354 & 5.70 \\
\hline 0.473 & 18.78 & 0.126 & 4.02 & 0.372 & 6.06 \\
\hline 0.484 & 19.44 & 0.148 & 5.10 & 0.408 & 6.70 \\
\hline 0.504 & 19.90 & 0.173 & 5.58 & 0.425 & 7.02 \\
\hline 0.515 & 20.14 & 0.218 & 7.04 & 0.462 & 7.50 \\
\hline 0.528 & 20.38 & 0.241 & 8.34 & 0.484 & 8.48 \\
\hline 0.577 & 22.12 & 0.277 & 8.92 & 0.504 & 9.02 \\
\hline 0.598 & 22.32 & 0.290 & 9.72 & 0.528 & 9.28 \\
\hline 0.620 & 23.38 & 0.333 & 10.46 & 0.555 & 9.50 \\
\hline 0.648 & 24.42 & 0.354 & 11.30 & 0.577 & 9.94 \\
\hline 0.692 & 25.72 & 0.386 & 12.06 & 0.648 & 12.66 \\
\hline 0.712 & 26.06 & 0.397 & 12.46 & 0.692 & 13.50 \\
\hline 0.787 & 28.74 & 0.408 & 12.98 & 0.712 & 14.14 \\
\hline 0.863 & 32.18 & 0.462 & 13.90 & 0.749 & 14.54 \\
\hline 0.884 & 33.26 & 0.484 & 15.22 & 0.787 & 15.30 \\
\hline 0.908 & 34.34 & 0.528 & 16.54 & 0.805 & 15.70 \\
\hline 0.936 & 35.14 & 0.565 & 17.10 & 0.824 & 15.90 \\
\hline 0.978 & 35.98 & 0.577 & 17.50 & 0.863 & 16.60 \\
\hline 0.999 & 36.56 & 0.631 & 18.76 & 0.884 & 16.92 \\
\hline Site 847 & & 0.681 & 20.24 & 0.908 & 17.60 \\
\hline 0.000 & 0.00 & 0.692 & 20.38 & 0.925 & 17.90 \\
\hline 0.056 & 1.11 & 0.749 & 21.92 & 0.978 & 18.80 \\
\hline 0.082 & 2.73 & 0.787 & 23.00 & 0.999 & 19.12 \\
\hline 0.103 & 3.47 & 0.805 & 23.24 & Site $\mathbf{8 5 2}$ & \\
\hline 0.126 & 4.11 & 0.853 & 24.56 & $\begin{array}{c}\text { Site } 852 \\
0.000\end{array}$ & 0.00 \\
\hline 0.219 & 7.65 & $\begin{array}{l}0.874 \\
0.908\end{array}$ & $\begin{array}{l}25.28 \\
26.16\end{array}$ & 0.056 & 0.73 \\
\hline 0.262 & 8.83 & $\begin{array}{l}0.908 \\
0.936\end{array}$ & $\begin{array}{l}26.16 \\
26.86\end{array}$ & 0.126 & 1.65 \\
\hline 0.290 & 9.79 & 0.957 & 27.36 & 0.196 & 2.31 \\
\hline 0.311 & 10.29 & 0.978 & 27.62 & 0.262 & 2.63 \\
\hline 0.333 & 11.13 & 0.999 & 28.26 & 0.290 & 3.03 \\
\hline 0.354 & 11.81 & 1.050 & 29.44 & 0.354 & 3.61 \\
\hline 0.372 & 12.23 & & & 0.408 & 4.21 \\
\hline 0.408 & 13.77 & Site 850 & & 0.462 & 4.67 \\
\hline 0.462 & 15.19 & 0.000 & 0.00 & 0.484 & 5.49 \\
\hline 0.484 & 16.05 & 0.056 & 1.03 & 0.577 & 6.31 \\
\hline 0.528 & 17.45 & 0.082 & 1.39 & 0.648 & 6.95 \\
\hline 0.577 & 18.23 & 0.126 & 2.31 & 0.692 & 7.85 \\
\hline 0.648 & 20.33 & 0.148 & 3.21 & 0.749 & 8.49 \\
\hline 0.692 & 21.47 & 0.173 & 3.89 & 0.787 & 9.05 \\
\hline 0.712 & 21.71 & 0.218 & 4.53 & 0.824 & 9.49 \\
\hline 0.749 & 22.69 & 0.262 & 5.45 & 0.863 & 10.09 \\
\hline 0.787 & 24.29 & 0.290 & 6.01 & 0.908 & 10.65 \\
\hline 0.824 & 25.15 & 0.333 & 6.95 & 0.957 & 11.17 \\
\hline 0.908 & 28.15 & 0.354 & 7.43 & 0.978 & 11.29 \\
\hline $\begin{array}{l}0.978 \\
1.000\end{array}$ & $\begin{array}{l}29.61 \\
30.43\end{array}$ & 0.372 & 7.85 & & \\
\hline 1.000 & 30.43 & 0.408 & 8.67 & $\begin{array}{c}\text { Site } 853 \\
0.00\end{array}$ & 0.22 \\
\hline Site 848 & & 0.425 & 9.07 & $\begin{array}{l}0.00 \\
0.78\end{array}$ & 2.95 \\
\hline 0.000 & 0.00 & $\begin{array}{l}0.445 \\
0.462\end{array}$ & $\begin{array}{l}9.41 \\
9.71\end{array}$ & 0.99 & 3.85 \\
\hline 0.056 & 0.68 & $\begin{array}{l}0.462 \\
0.484\end{array}$ & $\begin{array}{r}9.71 \\
10.39\end{array}$ & & \\
\hline 0.082 & 1.02 & $\begin{array}{l}0.404 \\
0.504\end{array}$ & 10.51 & Site 854 & \\
\hline 0.114 & 1.44 & 0.528 & 10.85 & 0.00 & 0.00 \\
\hline 0.148 & 2.14 & 0.577 & 11.59 & 0.78 & 4.08 \\
\hline 0.173 & 2.46 & 0.598 & 12.11 & 0.99 & 5.35 \\
\hline
\end{tabular}

Table 13. Coherency between June insolation at $65^{\circ} \mathrm{N}$ (Berger and Loutre, 1991) and stacked GRAPE density for sites 849,850 and 851 estimated over 1 m.y. intervals (Fig. 9).

\begin{tabular}{cccc}
$\begin{array}{c}\text { Interval } \\
(\mathrm{Ma})\end{array}$ & $\begin{array}{c}\mathrm{COH} 41 \\
\text { k.y. }\end{array}$ & $\begin{array}{c}\mathrm{COH} 23 \\
\text { k.y. }\end{array}$ & $\begin{array}{c}\mathrm{COH} 19 \\
\text { k.y. }\end{array}$ \\
\hline $0-1^{\mathrm{a}}$ & 0.87 & 0.63 & 0.38 \\
$0-1^{\mathrm{b}}$ & 0.89 & 0.91 & 0.78 \\
$1-2$ & 0.78 & 0.96 & 0.94 \\
$2-3$ & 0.89 & 0.96 & 0.90 \\
$3-4$ & 0.86 & 0.97 & 0.97 \\
$4-5$ & 0.74 & 0.92 & 0.91 \\
$5-6$ & 0.61 & 0.94 & 0.92 \\
$6-7$ & 0.42 & 0.90 & 0.91 \\
\hline \multicolumn{5}{c}{} \\
a ${ }^{8}$ O-based time scale from Tables 6, 7, and 8. \\
b GRAPE-based time scale from Table 12.
\end{tabular}

extremes. From a statistical standpoint, the tuning illustrated in Figure 2 leads to an acceptable coherency between GRAPE density and orbital insolation, whereas the tuning illustrated in Figure 1 does not (Table 13). The fact that neither version of the time scale for the interval from 0 to $1 \mathrm{Ma}$ leads to high coherency for both GRAPE density and $\delta^{18} \mathrm{O}$ has the unfortunate consequence that we cannot obtain statistically useful information on the phase relationship between these two parameters.

Between 1 and $2 \mathrm{Ma}$, the situation is slightly clearer (Fig. 3). In this time interval, $\delta^{18} \mathrm{O}$ records are dominated by $40-\mathrm{k} . \mathrm{y}$. obliquity cycles (Pisias and Moore, 1981; Ruddiman et al., 1986). Several segments of GRAPE density variability show evidence of 40-k.y. cycles over this interval; see, for example, Sites 847 and 852 in Figure 1A. The individual holes of Site 851 do not show the 40-k.y. cycles so clearly (Fig. 3), and it would not have been possible to develop the time scale on the basis of only this site.

Moving to the interval between 2 and $4 \mathrm{Ma}$ shown in Figure 1B, the tuning operation became easier. For intervals between 2.0 and 2.6 $\mathrm{Ma}$, we have been guided by the $\delta^{18} \mathrm{O}$ record of Site 846 (Shackleton et al., this volume). Correlating this record to that of Site 677 (SBP90) implies a strong obliquity signal in the GRAPE density, especially between 2.4 and $2.6 \mathrm{Ma}$. In some sites, the precession cycles between 2.1 and $2.3 \mathrm{Ma}$ are recognizable. The good magnetostratigraphic record for Site 851 (Fig. 4) provides a tie to the astronomical calibration of SBP90 in Site 677, and it is only for the section older than 2.6 Ma that we are seeking a tuning that is independent of previous work. Thus, the marked similarity between GRAPE density variations and the orbital record between 2.5 and $3.0 \mathrm{Ma}$ in several sites, as well as the conspicuous precession cycles in the interval from 3.0 and $3.2 \mathrm{Ma}$ and between 3.7 and $4.0 \mathrm{Ma}$, are particularly important for carrying the tuning operation back through the Gauss. It is appropriate to remark that our starting point was the assumption that since the time scale developed by Cande and Kent (1992) was calibrated astronomically at $2.6 \mathrm{Ma}$, it would prove to be nearly correct. We were aware that this time scale diverges from $\mathrm{H} 91$ in the Gilbert Chron, but imagined (wrongly, as it turns out) that this disagreement would be resolved in favor of smooth seafloor spreading and, hence, in favor of the time scale of Cande and Kent (1992). The data for the individual holes of Sites 850 and 851 for the interval 3 to $4 \mathrm{Ma}$ are shown in Figures 5 and 6. From 3.0 to $3.5 \mathrm{Ma}$, the tuning is exceptionally clear; however, with the data from several sites to work with, the tuning to $4 \mathrm{Ma}$ also is reliable.

The interval from 4.0 to $6.0 \mathrm{Ma}$ is shown in Figure 1C. Again, the precession signal is well recorded in several of the sites. Between 4.0 and $4.4 \mathrm{Ma}$, Site 846 shows a clear precession signal, and both Sites 846 and 847 appear to record the interval between 4.6 and 5.0 Ma, during which the insolation record shows strong precession cycles flanking an interval dominated by obliquity. Of course, this pattern is a reflection of eccentricity maxima flanking a broad interval of low eccentricity. The significance of this is that although uncertainties in the astronomical calculations mean that the exact temporal relation- 


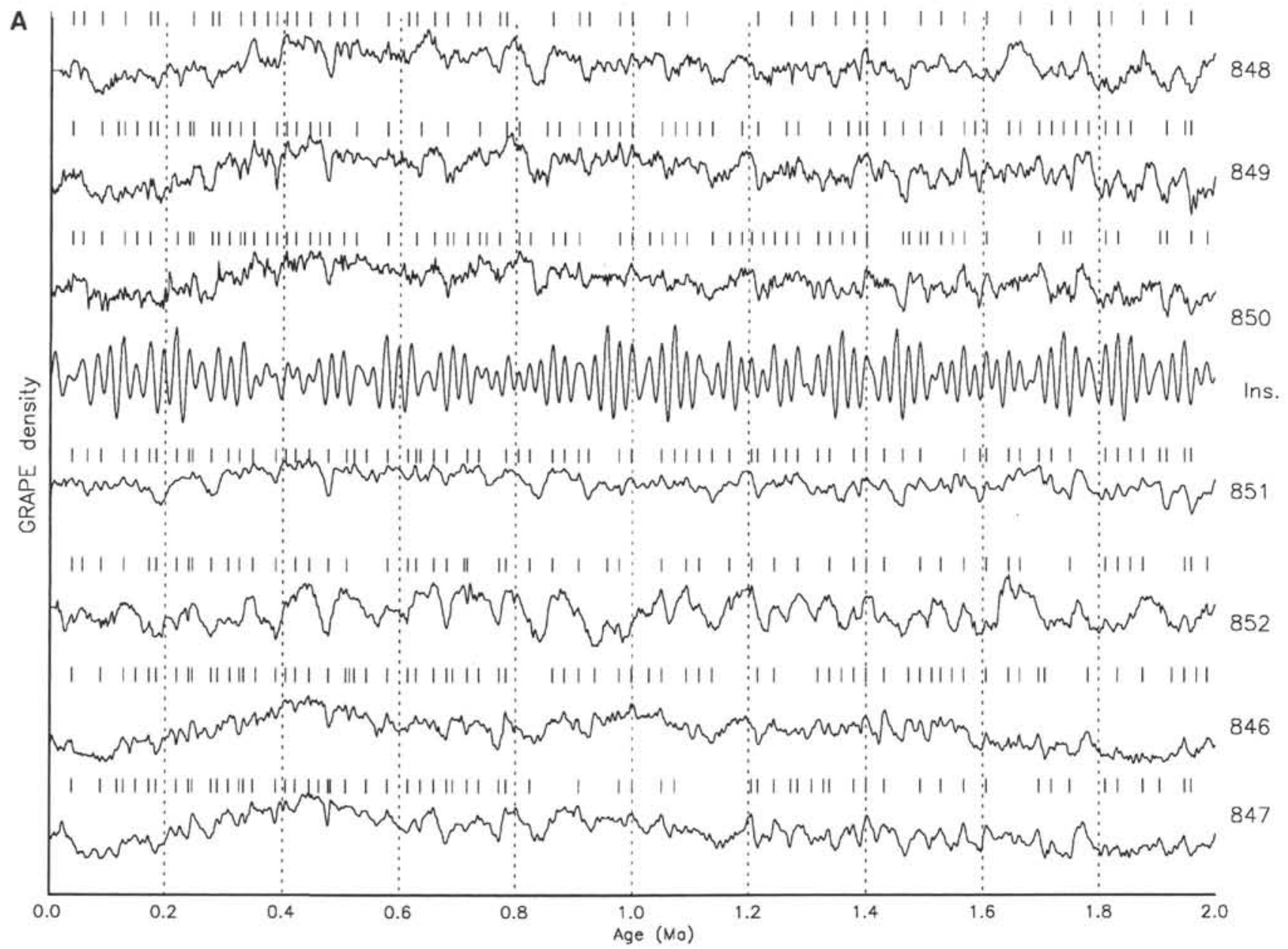

Figure 1. A. GRAPE density vs. age for Sites $846,847,848,849,850,851$, and 852,0 to $2 \mathrm{Ma}$. For each site, the upper part has been constructed from the stacked records, making use of data from all holes at the site, while the older parts of the sections have been based on the shipboard splice. Vertical lines above the data for each site show the age control points from Tables 1 to 11. For this figure, all data sets have been smoothed in the time domain with the same filter (see text). B. GRAPE density vs. age for Sites $846,847,848,849,850,851$, and 852,2 to $4 \mathrm{Ma}$. C. GRAPE density vs. age for Sites $846,847,848,849,850,851$, and 852 , 4 to 6 Ma. D. GRAPE density vs. age for Sites $846,847,848,849,850,851$, and 852,6 to 8 Ma. E. GRAPE density vs. age for Sites $846,847,848,849,850,851$, and 852,8 to $10 \mathrm{Ma}$. F. GRAPE density vs. age for Sites $846,847,848,849,850,851$, and 852,10 to $12 \mathrm{Ma}$.

ship between a particular precession peak and a particular obliquity maximum may be unknown, the timing of the eccentricity record is probably reliable (Berger et al., 1992).

Between 5 and $6 \mathrm{Ma}$, GRAPE density variations are more erratic, but even so, there appear to be intervals having large-amplitude variations associated with precession. These large jumps in mean density value adversely affect the results of bandpass filtering of the data. Their origin is partly the episodes during which laminated sediments accumulated (Kemp and Baldauf, 1993). It is difficult to put bounds on possible sedimentation rate excursions associated with these events and, in some details, the tuning is speculative in those parts of the record associated with accumulation of laminated sediments. At about $5.8 \mathrm{Ma}$, we were assisted in correlating sites by features in the bulk sediment $\delta^{13} \mathrm{C}$ record that could be correlated among sites (Shackleton and Hall, this volume).

\section{STATISTICAL EVALUATION}

To present a straightforward statistical evaluation of the time scales that have been generated, we constructed a synthetic western transect record by simply averaging the GRAPE density estimate at each 0.001 - Ma age increment at Sites 849,850 , and 851. Figure 9 shows cross-spectral analyses of this record vs. the $65^{\circ} \mathrm{N}$ insolation record of Berger and Loutre (1991) in million-year segments. It is apparent from Figure 9 that tuning has resulted in coherency estimates in the precession band of more than 0.9 in every time interval except 0 to $1 \mathrm{Ma}$. Coherency estimates are given in Table 13. In every time interval, coherency with precession is greater than coherency with obliquity; coherency with obliquity ranges from a low of 0.61 in the 5- to 6-Ma interval to 0.89 in the 2- to 3 -Ma interval. Phase plots are not shown because we tuned by assuming a zero phase lag between insolation and GRAPE density; however, note that in no case are the phase estimates for either precession or obliquity significantly different from zero, other than the phase against obliquity in the range of from 3 to $4 \mathrm{Ma}$, where GRAPE density lags insolation by $50 \pm 20^{\circ}$ ( $6 \pm 2$ k.y.) in the obliquity band. In the interval from 0 to $1 \mathrm{Ma}$, coherency is acceptable for the age models in which GRAPE density extremes are exactly aligned with insolation extremes (Table 12), but not for the $\delta^{18} \mathrm{O}$ age models given in the upper parts of Tables 1 to 11 .

Coherency between the geological data and the orbital target in the precession band is the fundamental method by which a time scale may be evaluated. There are two reasons for this. First, the modulation on the precession signal is very much stronger than that on the obliquity signal, so that the test of coherency is more valuable. Second, the modulation on the precession signal arises directly from the orbital eccentricity record for which the calculations are the most robust (Berger et 


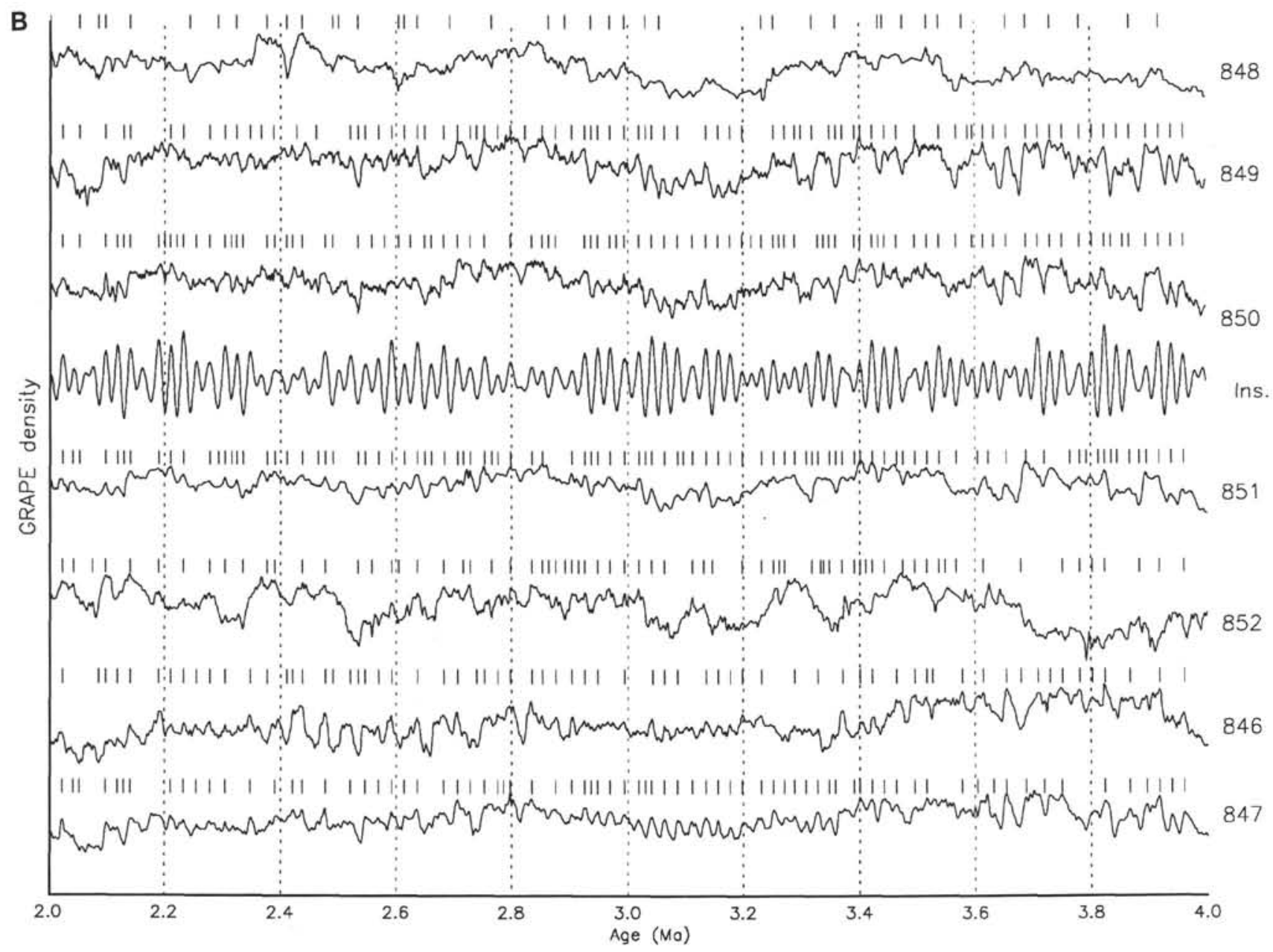

Figure 1 (continued).

al., 1992), whereas the modulation on the obliquity signal (1) does not have a clearly defined and independent origin, (2) appears in the series expansion through the interference between several terms with periods close to 41 k.y. (Berger and Loutre, 1991; Table 7), and (3) thus is sensitive to extremely small errors in their estimation.

Two studies (Pisias, 1983, and Brüggemann, 1992) showed that high coherencies cannot be generated by "tuning" a randomly varying time series to the orbital signal (although Neeman [in press] reached a different conclusion). Thus, it seems unlikely that the very high coherencies shown in Figure 9 could have been obtained had there not been a close coupling between changing solar insolation and East Pacific Ocean paleoceanography. Moreover, Hagelberg (1993) showed that, although estimated coherency is reasonably robust with respect to small errors in time scale, the reduction in coherency resulting from time-scale error is frequency dependent, with the coherency at precession frequencies showing the highest degradation. Thus, the very high coherencies in the precession band shown in Figure 9 constitute strong evidence that our time scale is close to correct. It must be pointed out that the high coherencies shown by Imbrie et al. (1984) for a stacked $\delta^{18} \mathrm{O}$ record covering the past 0.78 Ma did also imply that the chronology was close to correct; this remains true, despite the fact that we now think that it was correct only over $75 \%$ of the interval covered (SBP90).

The high coherencies shown in Figure 9 also indicate that the physical linkage between changing solar insolation and paleoceanography has remained strong through the whole Pliocene, suggesting that it may be amenable to modeling. On the other hand, it must be said that it is important in the future to test the validity of the calibrations that we have obtained beyond the range of overlapping with $\mathrm{H} 91$ in an area that experienced less violent fluctuations in sedimentation rate. The reason is that one property of a convincing age model is that it should not generate physically unreasonable changes in sedimentation rate; one of the findings of Leg 138 was that in the eastern equatorial Pacific, sedimentation rates are extremely variable so that it is difficult to specify what is, in fact, a physically unreasonable change. In addition, the hole-to-hole comparisons made by Hagelberg et al. (this volume) show that a significant proportion of the apparent variability in sedimentation rate either persists over only small distances on the seafloor or else is an artifact of distortion during coring.

It is a striking feature of both the records from the Mediterranean Basin, studied by Hilgen (1991a, 1991b), and those from the eastern Atlantic Ocean, studied by Tiedemann (1992), that good evidence can be seen for a 100-k.y. eccentricity cycle in their data. Indeed, the ground-breaking study by Hilgen (1991a) was possible only because he was able to place his records in the context of the 400- and 100-k.y. eccentricity cycles and so could to develop an astronomically calibrated time scale without working systematically back from the present. It is evident from Figures 1 and 9 that this approach is not possible in the GRAPE density records recovered during Leg 138. No consistent 100-k.y. signal is present, and coherency between insolation and GRAPE density is only marginally significant in the eccentricity fre- quency band. Figure 1 shows that there is considerable low-frequency variability in GRAPE density; presumably, this masks any eccentricity signal that might otherwise have been present. 


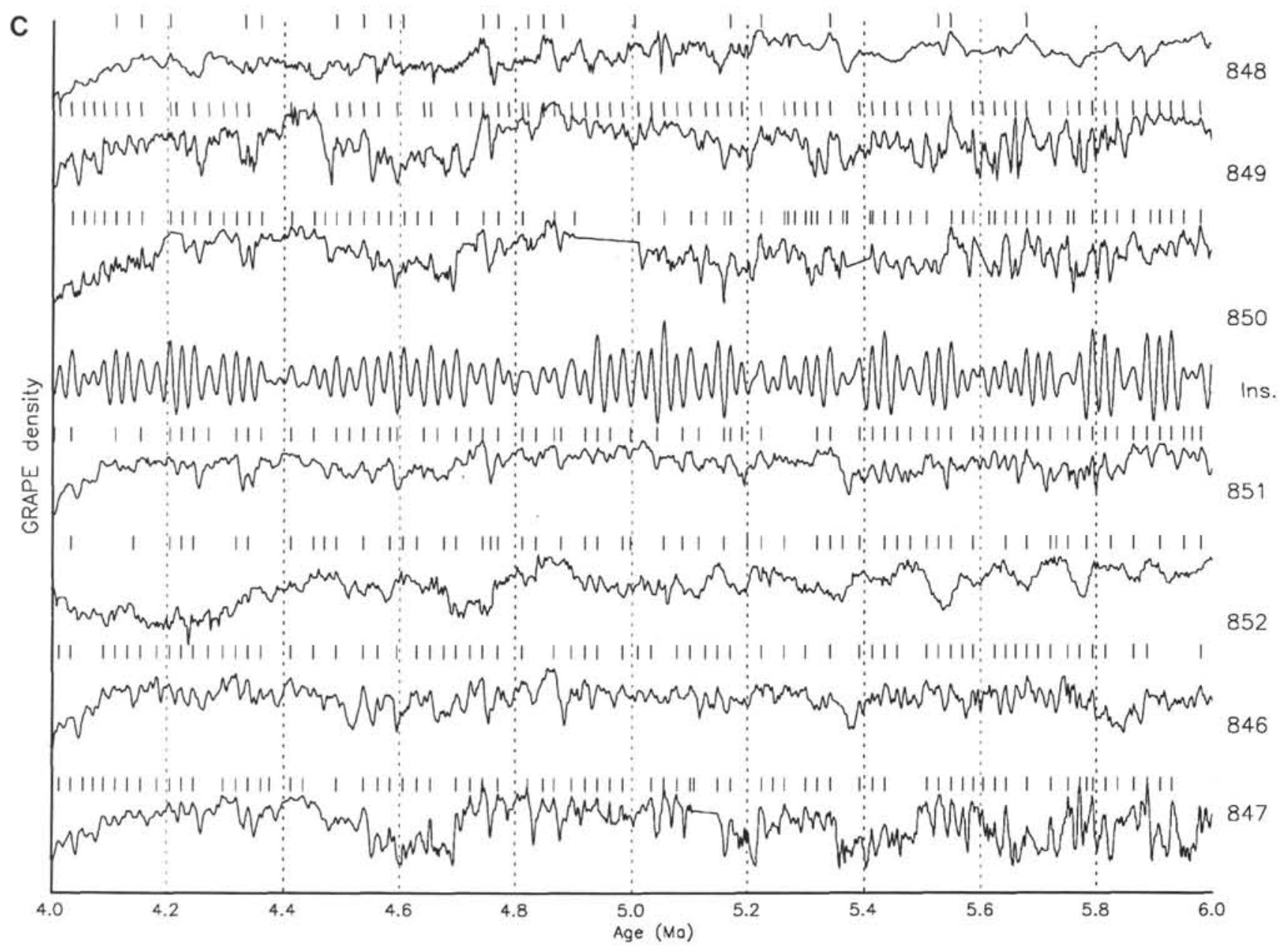

Figure 1 (continued).

\section{DISCUSSION: CALIBRATIONS OF THE MAGNETIC POLARITY TIME SCALE FROM 0 TO 6 M.Y.}

To obtain the most reliable ages for Pliocene magnetostratigraphy, we examined the critical intervals hole by hole. This enabled us to evaluate the quality of each estimate. In Figures 2 to 5 , we show the magnetic declination and tuned GRAPE density vs. age for each hole in Sites 850 and 851 over the interval from 3 to $4 \mathrm{Ma}$. For the Kaena and Mammoth subchrons, the boundaries are clearly related to the GRAPE stratigraphy, and the estimates are consistent (Table 14). Initially, we were unable to obtain a clear calibration for the base of the Gauss, but careful examination of the data for Holes $850 \mathrm{~A}$ and $850 \mathrm{~B}$ (Fig. 5) shows clear precession cycles; by correlating the holes of Site 851 to those of Site 850, we obtain a consistent calibration.

In the Gilbert interval, Site 852 provides the vehicle for transferring our time scale to the paleomagnetic record; in addition, a record of the Cochiti occurs in Site 851. Figures 7 and 8 show the GRAPE density and magnetic declination data for the individual holes of Site 852 over the intervals from 4 to $5 \mathrm{Ma}$ and from 5 to $6 \mathrm{Ma}$. In these records, the age control points derive from correlation to Sites 849 , 850 , and 851 , rather than direct correlation to the orbital record; for this reason, we show in Figures 7 and 8 the stacked GRAPE density records of Sites 849,850 , and 851 , as well as the orbital insolation, so that the tuning process may be followed. The interpolated ages of each of the reversals in each hole are given in Table 15.

The top of the Nunivak is a problematic area because the $65^{\circ} \mathrm{N}$ orbital signature is structureless where the GRAPE data show evident structure; here, we may have difficulties with the accuracy of the astronomical solution. If this is the correct explanation, we would be led to conclude that the eccentricity values were not so low as those given in the calculations of Berger and Loutre (1992). Such a conclusion would not necessarily be in conflict with the observation that the main eccentricity periods are known rather accurately so that the timing of eccentricity maxima is reliably known at ages where the timing of obliquity maxima is less well known.

Table 16 provides mean estimates for the ages of the magnetic reversals in Sites 850,851, and 852 that arise from the tuning discussed above. These estimates are compared (1) with those that, until recently, have been regarded as "standard" (Berggren, Kent, and Flynn (1985) and Berggren, Kent, and Van Couvering (1985); (2) with those given by CK92; and (3) with the earlier tuned ages of SBP90 and H91.

It is apparent from Table 16 that ages for the Kaena and Mammoth intervals agree well with both Hilgen's estimates and those of CK92. At the base of the Gauss, our estimate agrees with that of Hilgen (1991a); both estimates are significantly older than those of CK92. The age obtained here, $3.594 \mathrm{Ma}$, is a little younger than that reported by Shackleton et al. (1992). The reason is that we reevaluated the GRAPE density record of Site 850, which has the higher sedimentation rate across this interval, and identified the complete sequence of precession cycles in this critical interval. We then mapped the Site 851 holes into this record. The result (Figs. 5 and 6 ) is convincing.

On average, our age estimates are a few thousand years greater than those given by Hilgen (1991a). Hilgen's estimates were based on an assumed lag of 4 k.y. between precession extremes and the midpoints of the equivalent lithological bed, whereas we have not assumed any lag between insolation and GRAPE density extremes. 


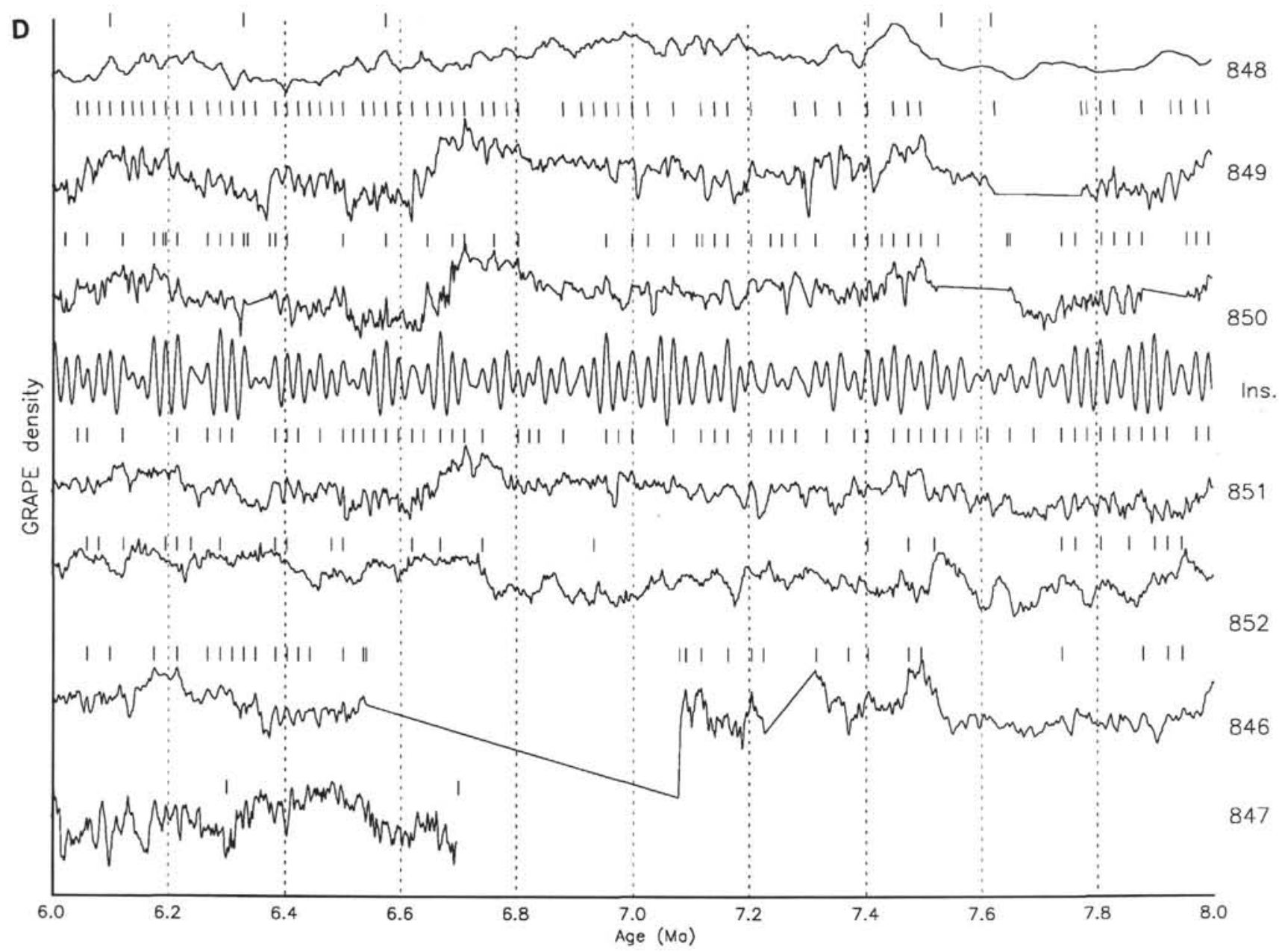

Figure 1 (continued).

Pending a more sophisticated evaluation of the response of the respective paleoceanographic systems to orbital forcing, we conclude that differences between our estimates and $\mathrm{H} 91$ are negligible. From the Cochiti to the base of the Thvera subchrons, our estimates also are near those given by Hilgen (1991b), although, because they were calibrated in more slowly accumulating sediment, our estimates for the ages of these reversals are not so precise as those for the reversals in the Gauss. However, it is now clear that this time scale provides true accuracy through a sufficient amount of the Pliocene that one must recalibrate the magnetic anomaly time scale of $\mathrm{CK} 92$ to take into account the significant deviations that become evident by the base of the Gauss.

From the Thvera to C3A.1n (t), tuning was difficult as a result of the exceptionally wide ranges in sedimentation rate in several sites. However, we attempted it for two reasons. First, this is a key to extending the work of Hilgen (1991b) into the Miocene: it is necessary to cover the interval of the Messinian salinity crisis by working in extra-Mediterranean sediments. Second, the young side of $\mathrm{C} 3 \mathrm{~A}$ is widely used as a calibration point when developing time scales for the seafloor magnetic anomaly sequence. In the interval from 5 to $6 \mathrm{Ma}$, GRAPE density and orbital insolation are highly coherent in the precession band, but only weakly coherent in the obliquity band (Fig. 9F); moreover, in our solution, no discrete concentration of variance is observed in the 41-k.y. band. The $\delta^{18} \mathrm{O}$ record developed for Site 846 does not show a strongly coherent 41-k.y. signal either; it remains possible that either a different tuning of the data investigated here or a record of different components of the climate system might lead to higher coherencies than those we have obtained here.

\section{RECALIBRATING THE MIOCENE TIME SCALE}

One will recall that CK92 calibrated the distance scale for the South Atlantic Ocean anomaly sequence on the basis of a control age at 2.6 $\mathrm{Ma}$ and another at $14.8 \mathrm{Ma}$ (and of course others through the past 100 Ma). The age of $2.6 \mathrm{Ma}$ for the Gauss/Matuyama boundary was based on astronomical calibration (SBP90), while the remaining have been based on radiometric age determinations. Clearly, for several years to come, there will be two sections to the anomaly time scale: an upper section that is calibrated in detail by astronomical tuning, and a lower section that is developed by interpolation between a limited number of control points that are based on radiometric dates. A possible procedure at this juncture would be to insert one new age control in the latest Miocene and retain the remaining points as used by CK92. However, when South Atlantic spreading rates are estimated on this basis, a geophysically unexpected oscillation in spreading rate is generated (Fig. 10). Therefore, we have inserted an additional control point on the basis of the new determination by Baksi (1992) for C5 (t), $9.66 \pm$ $0.05 \mathrm{Ma}$. For ease of use, we have adopted the value of $9.64 \mathrm{Ma}$ for this boundary, which is the closest age within the uncertainty limits that enables GRAPE density to be matched directly to the insolation record. We have used $5.875 \mathrm{Ma}$ for $\mathrm{C} 3 \mathrm{~A}(\mathrm{t})$. Table 17 gives the ages of reversal boundaries estimated from Table 2 in CK92 by fitting a cubic-spline in the same manner as they adopted. If one uses these new figures for events younger than $14.8 \mathrm{Ma}$ together with those given by CK92 for older events, this does not generate a significant discontinuity at 14.8 $\mathrm{Ma}$; we suggest that this time scale is probably more nearly correct as regards its depiction of changes in spreading rate during the late 


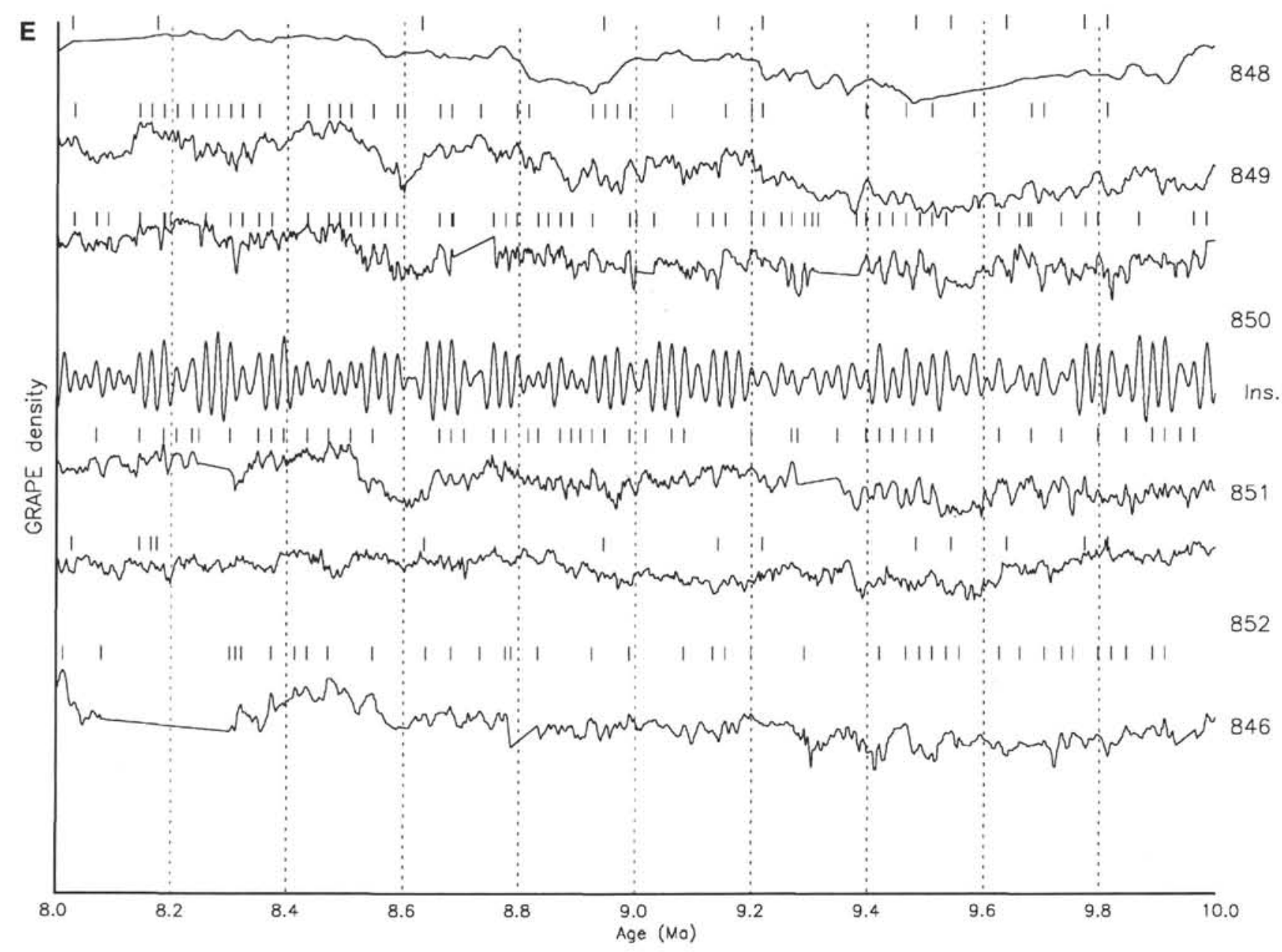

Figure 1 (continued).

Neogene (Fig. 10) than that of CK92. Because South Atlantic spreading rates clearly did change significantly during the late Miocene, it is highly likely that future tuning will modify this picture. These future modifications may undermine the basis for using a cubic-spline fit to predict the ages of the seafloor anomalies, but in the meantime, we recommend that the ages in Table 17, together with those in CK92, Table 6, for ages greater than $14.8 \mathrm{Ma}$, be used for Miocene calibrations. We are aware that the control age at $14.8 \mathrm{Ma}$ may also be questioned (Baksi and Farrar, 1990) but prefer here to devise a solution that limits the adjustments recommended to that part of the time scale over which we have contributed new data.

\section{DISCUSSION: MIOCENE AGES}

Our objective was to generate an accurate, high-resolution time scale for the past 6 m.y. for Leg 138 sites. Several factors limit the backward extension of this type of time scale. First, the quality of our data deteriorates. It is not yet clear to what extent our composite depth sections are complete representations of the sediment column where the extended core barrel (XCB) was used instead of the APC. Second, both the quality of the GRAPE density data and the fidelity of its relationship to percentage carbonate deteriorates in more lithified sediments. Third, sedimentation rates are not so favorable in the mid-portion of the late Miocene sequence. Fourth, Berger and Loutre (1991) did not claim accuracy for their astronomical reconstructions prior to about $5 \mathrm{Ma}$ and, indeed, it has already been suggested that modifications in the calculations may be required (Berger and Loutre, 1992). Fortunately, the chief basis for tuning is the characteristic
Table 14. Ages for reversals in the mid-Pliocene, estimated for Sites $\mathbf{8 5 0}$ and 851 .

\begin{tabular}{|c|c|c|c|c|c|c|c|}
\hline Event & $\begin{array}{l}\text { Hole } \\
850 \mathrm{~A}\end{array}$ & $\begin{array}{l}\text { Hole } \\
850 \mathrm{~B}\end{array}$ & $\begin{array}{l}\text { Hole } \\
851 \text { B }\end{array}$ & $\begin{array}{l}\text { Hole } \\
851 \mathrm{C}\end{array}$ & $\begin{array}{l}\text { Hole } \\
851 \mathrm{E}\end{array}$ & Mean & Event \\
\hline$C 2 n, 1 r(t)$ & 3.043 & 3.048 & 3.044 & N.D. & 3.049 & 3.046 & Kaena (t) \\
\hline $\mathrm{C} 2 \mathrm{n} .1 \mathrm{r}(\mathrm{o})$ & 3.133 & 3.133 & 3.124 & 3.132 & 3.131 & 3.131 & Kaena (o) \\
\hline $\mathrm{C} 2 \mathrm{n} \cdot 2 \mathrm{r}(\mathrm{t})$ & 3.224 & 3.219 & 3.230 & 3.222 & 3.233 & $3.233^{\mathrm{a}}$ & Mammoth (t) \\
\hline $\mathrm{C} 2 \mathrm{n} \cdot 2 \mathrm{r}(\mathrm{o})$ & 3.333 & 3.329 & 3.327 & 3.331 & 3.335 & 3.331 & Mammoth (o) \\
\hline $\mathrm{C} 2 \mathrm{n}(\mathrm{o})$ & 3.595 & 3.596 & 3.594 & 3.592 & 3.591 & 3.594 & Gauss (o) \\
\hline$C 3 n \cdot \ln (t)$ & N.D. & N.D. & 4.194 & 4.208 & 4.208 & $* *$ & Cochiti (t) \\
\hline C $3 n . \ln (o)$ & N.D. & N.D. & 4.322 & N.D. & 4.320 & ** & Cochiti (o) \\
\hline
\end{tabular}

a At C2n.2r(t), the GRAPE density signal is much clearer at Hole 851E than at the other holes, so that we have taken the estimate for that hole, rather than the mean. $(t)=$ termination and $(o)=$ onset. ${ }^{* *}$ See Table 15 .

modulation of the precession signal by eccentricity, while the most likely modification to the calculated record would be in the timing of the obliquity cycles with respect to the precession cycles.

On the positive side, several of the Leg 138 sites have good magnetostratigraphy, all have good biostratigraphy, and the GRAPE density records show considerable promise. Thus, we have aimed to develop partially tuned age models for the interval between 6 and 10 $\mathrm{Ma}$. Their chief practical utility is that they enable us to propose detailed correlation among sites wherever the GRAPE data permits it; they also enable us to propose calibrated sedimentation rates over intervals that display orbital frequency variability; and finally, they enable us to evaluate the changing response to orbital forcing. We have made some use of bulk sediment $\delta^{13} \mathrm{C}$ data (Shackleton and Hall, this volume) as an additional tool for correlation between sites. 


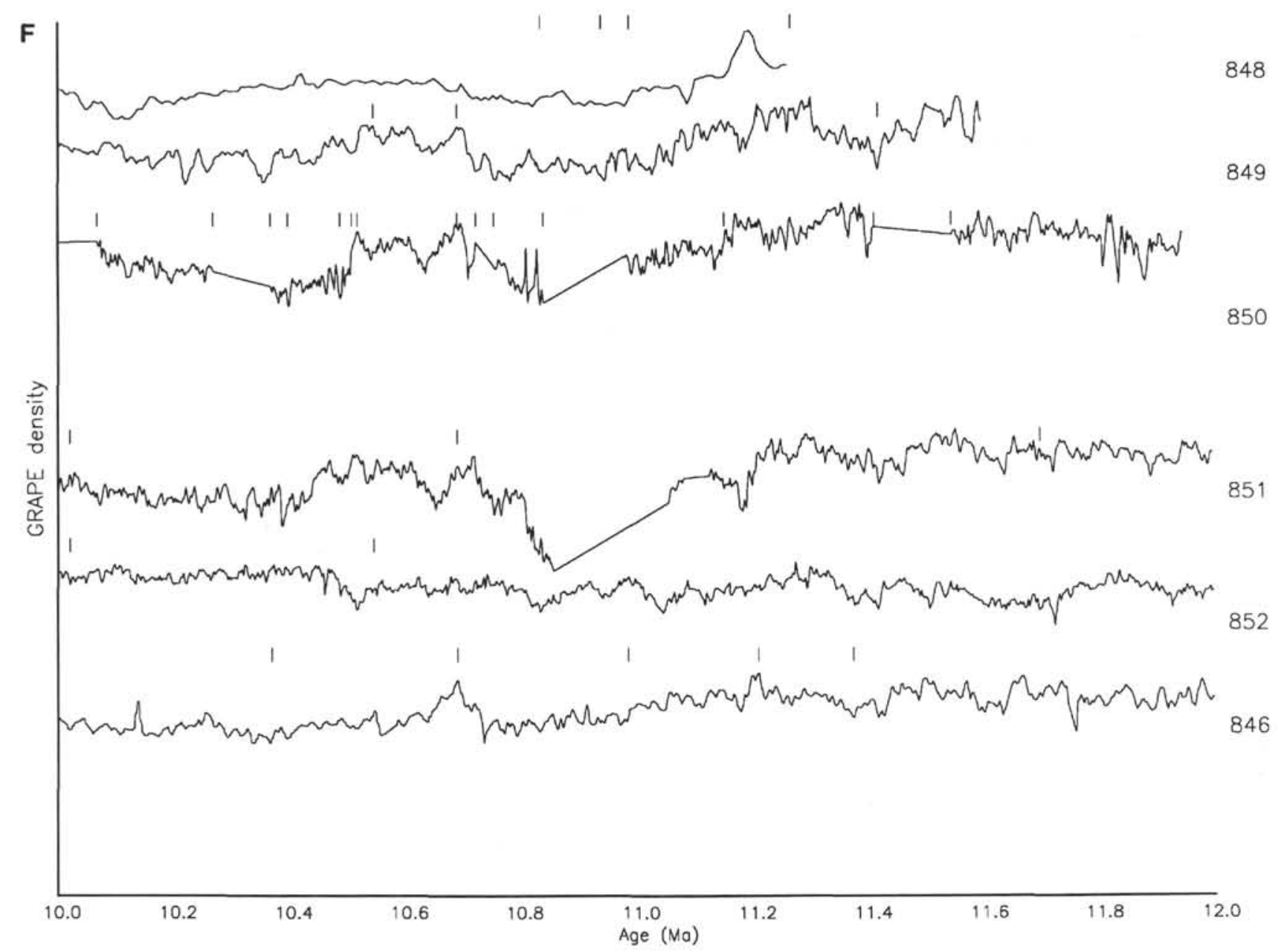

Figure 1 (continued).

Table 15. Ages for reversals in the Gilbert, estimated for Site $\mathbf{8 5 2}$.

\begin{tabular}{|c|c|c|c|c|c|}
\hline & Hole & Hole & Hole & & \\
\hline Event & $852 \mathrm{~B}$ & $852 \mathrm{C}$ & $852 \mathrm{D}$ & Mean & Event \\
\hline$C 3 n \cdot \ln (t)$ & 4.197 & 4.194 & 4.194 & $4.1992^{\mathrm{a}}$ & Cochiti (t) \\
\hline C3n. $\ln (\mathrm{o})$ & 4.307 & N.D. & 4.316 & $4.316^{\mathrm{a}}$ & Cochiti (o) \\
\hline$C 3 n \cdot 2 n(t)$ & N.D. & 4.480 & 4.478 & 4.479 & Nunivak ( $t$ ) \\
\hline C $3 n .2 n(o)$ & 4.621 & 4.618 & 4.631 & 4.623 & Nunivak (o) \\
\hline C3n.3n(t) & 4.780 & 4.785 & 4.777 & 4.781 & Sidufjall (t) \\
\hline C3n.3n(o) & 4.882 & 4.875 & 4.877 & 4.878 & Sidufjall (o) \\
\hline $\mathrm{C} 3 \mathrm{n}, 4 \mathrm{n}(\mathrm{t})$ & 4.980 & 4.975 & 4.977 & 4.977 & Thvera $(t)$ \\
\hline $\mathrm{C} 3 \mathrm{n} .4 \mathrm{n}(\mathrm{o})$ & 5.240 & 5.224 & 5.231 & 5.232 & Thvera (o) \\
\hline C3A. In $(t)$ & 5.870 & 5.892 & 5.885 & 5.882 & Epoch $5(t)$ \\
\hline C 3 A. $\ln (\mathrm{o})$ & 6.106 & 6.110 & N.D. & 6.108 & \\
\hline C $3 A \cdot 2 n(t)$ & 6.275 & 6.277 & 6.283 & 6.278 & \\
\hline
\end{tabular}

"at $\mathrm{C} 3 \mathrm{n} \cdot \ln (\mathrm{o})$ and $(t)$, the mean is based on Sites 851 and $852,(t)=$ termination and $(\mathrm{o})$ $=$ onset.

The interval between 6 and $8 \mathrm{Ma}$ is shown in Figure 1D. Between 6 and $7 \mathrm{Ma}$, several sites show variability that is readily tuned to the insolation record, and some preliminary tuning has been performed. Note that the GRAPE density minimum between 6.5 and 6.6 Ma can be traced from Site 853 with a complete paleomagnetic record, through Site 852 with a similar GRAPE density record, to the extreme represented by Site 850 , where this interval is marked by a 20 -m-thick sequence of laminated sediment.

In the interval from 8 to $10 \mathrm{Ma}$ shown in Figure 1E, further work will be required to ensure the continuity of the records recovered with the XCB system, but it may be possible ultimately to generate a
Table 16. Ages of the magnetic reversals of the past 6 m.y., according to SBP90, H91, CK92, and this study.

\begin{tabular}{|c|c|c|c|c|c|}
\hline Event & SBP90 & H9l & CK92 & This study & Event \\
\hline $\mathrm{C} \ln (\mathrm{o})$ & 0.78 & & 0.780 & & $\mathrm{~B} / \mathrm{M}$ \\
\hline$C \operatorname{lr} \cdot \ln (t)$ & 0.99 & & 0.984 & & Jaramillo (t) \\
\hline C1r. $\ln (0)$ & 1.07 & & 1.049 & & Jaramillo (o) \\
\hline $\mathrm{C} 1 \mathrm{r} \cdot 2 \mathrm{n}(\mathrm{t})$ & 1.77 & & 1.757 & & Olduvai (t) \\
\hline C1r.2n (o) & 1.95 & & 1.983 & & Olduvai (o) \\
\hline $\mathrm{C} 2 \mathrm{An} \cdot \ln (\mathrm{t})$ & 2.60 & $2.59 / 62$ & 2.600 & & Gauss (t) \\
\hline $\mathrm{C} 2 \mathrm{An} \cdot \ln (\mathrm{o})$ & & 3.04 & 3.054 & 3.046 & \\
\hline C $2 A n \cdot 2 n(t)$ & & 3.11 & 3.127 & 3.131 & \\
\hline $\mathrm{C} 2 \mathrm{An} \cdot 2 \mathrm{n}(\mathrm{o})$ & & 3.22 & 3.221 & 3.233 & \\
\hline C2An.3n (t) & & 3.33 & 3.325 & 3.331 & Mammoth (o) \\
\hline $\mathrm{C} 2 \mathrm{An} \cdot 3 \mathrm{n}(\mathrm{o})$ & & 3.58 & 3.553 & 3.594 & Gilbert ( $t$ ) \\
\hline $\mathrm{C} 3 \mathrm{n} . \ln (\mathrm{t})$ & & 4.18 & 4.033 & 4.199 & Cochiti (t) \\
\hline $\mathrm{C} 3 \mathrm{n} \cdot \ln (\mathrm{o})$ & & 4.29 & 4.134 & 4.316 & Cochiti (o) \\
\hline$C 3 n \cdot 2 n(t)$ & & 4.48 & 4.265 & 4.479 & Nunivak (t) \\
\hline C.3n.2n (o) & & 4.62 & 4.432 & 4.623 & Nunivak (o) \\
\hline$C 3 n .3 n(t)$ & & 4.80 & 4.611 & 4.781 & Sidufjall ( $(t)$ \\
\hline$C 3 n .3 n(0)$ & & 4.89 & 4.694 & 4.878 & Sidufjall (o) \\
\hline $\mathrm{C} 3 \mathrm{n} \cdot 4 \mathrm{n}(\mathrm{t})$ & & 4.98 & 4.812 & 4.977 & Thvera (t) \\
\hline C $3 n .4 n(o)$ & & 5.23 & 5.046 & 5.232 & Thvera (o) \\
\hline $\mathrm{C} 3 \mathrm{An} \cdot \ln (\mathrm{t})$ & & & 5.705 & $5.882^{\mathrm{a}}$ & C3A.nl (t) \\
\hline $\mathrm{C} 3 \mathrm{An} \cdot \ln (\mathrm{o})$ & & & 5.946 & $6.108^{a}$ & C3A.nl (o) \\
\hline$C 3 A n \cdot 2 n(t)$ & & & 6.078 & $6.278^{\mathrm{a}}$ & C $3 A \cdot n 2(t)$ \\
\hline
\end{tabular}

"These values are regarded as tentative; we recommend using the figures in Table 17 until a more secure calibration is obtained for events in the late Miocene. $(t)=$ termination; (o) = onset. 

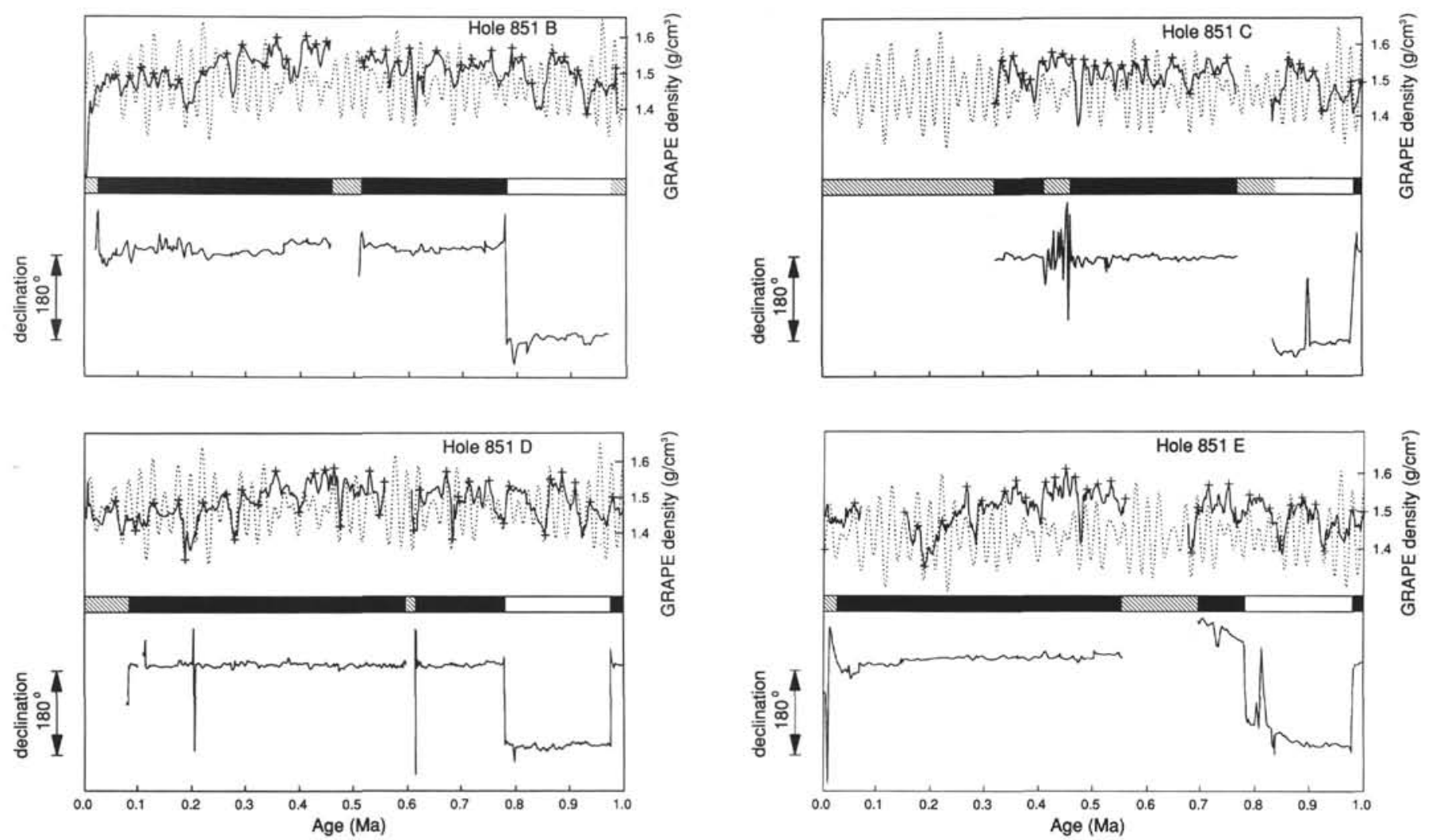

Figure 2. GRAPE density and magnetic declination for Holes $851 \mathrm{~B}, 851 \mathrm{C}, 851 \mathrm{D}$, and $851 \mathrm{E}$ for the interval 0 to $1 \mathrm{Ma}$, with orbital tuning target. Age control points are marked on the GRAPE density record. Declinations have been rotated arbitrarily for ease of comparison; the original data are shown in the site chapters in Mayer, Pisias, Janecek, et al. (1992).

continuous tuning to $10 \mathrm{Ma}$. In the meantime, we have made some preliminary correlations to the orbital record so that in correlating a particular maximum in the GRAPE density record from one site to another we use an age corresponding to an insolation maximum. It was on this basis that we adopted the value of $9.639 \mathrm{Ma}$ for C $5 \mathrm{n} .1 \mathrm{n}$ $(t)$, which is consistent with the estimate of $9.66 \pm 0.05$ Ma given by Baksi (1992), while permitting the tentative tuning close to that age shown in Figure 1E.

Beyond $10 \mathrm{Ma}$, no orbital estimates were available to us, although in fact, the calculations of Berger and Loutre (1991) have been extended back in time (Berger, pers. comm., 1993). We have made some use of GRAPE density as well as biostratigraphy to correlate the other sites to Site 845 , for which a good magnetostratigraphy is available to C5AB.n (t) at a recalibrated age of 13.252 Ma. Below that, we have not attempted here to improve on the shipboard age models, which were based exclusively on biostratigraphy. In Tables 1 to 11, we identify those age control points that are based on magnetostratigraphy or biostratigraphy, rather than on GRAPE density.

\section{SEDIMENTATION RATES}

The high sediment accumulation rates along the equator are the most obvious geological indication of the characteristics of the physical oceanography of the region, and scientists have long known that the paleoceanographic history could be partly sought simply by examining the history of changing sedimentation rates. The work of van Andel et al. (1975) elegantly exploited and reviewed the material that was available up to the time of DSDP Leg 17. Perhaps the most striking scientific achievement during Leg 138 was the production of the high quality biostratigraphy and magnetostratigraphy that enabled us to generate refined sedimentation rate history (Mayer, Pisias, and Shipboard Scientific Party [1992] and Shackleton and Shipboard
Scientific Party [1992]). The main feature of that result was the remarkably high sedimentation rates that prevailed over an interval of about 3 m.y. in the equatorial Pacific Ocean during the early Pliocene and latest Miocene.

Figure 11 shows the sedimentation rate picture that emerges from the more refined time scales developed in this chapter. For each site, sedimentation rate has been estimated over 0.2 -m.y. intervals centered at each $0.1 \mathrm{Ma}$ in age (Tables 18 to 28). This presentation effectively damps out any sedimentation rate variability that may be attributed to the result of Milankovitch-scale processes. Both the onset of the interval of enhanced sedimentation rates at about $7.5 \mathrm{Ma}$ and its termination at about $4.5 \mathrm{Ma}$ are surprisingly rapid. We suggest (1) that it is hardly likely that such dramatic changes in the eastern Pacific Ocean could occur without repercussion in other parts of the global climate system and (2) that efforts should be made to identify related changes in other regions with a view to identifying the cause. Certainly, analogous changes have been reported in the equatorial Indian Ocean (Peterson and Backman, 1990) as well as in the western equatorial Pacific (Berger et al., 1993).

\section{SUMMARY}

A consistent set of high-resolution age models for the Leg 138 sites is presented; these were provided to the Shipboard Scientific Party for use in preparing other chapters in this volume. For the past 6 m.y., these are fully orbitally tuned, providing a secure, absolute time scale for the seafloor anomaly scale, for the oxygen isotope record, for the seismic stratigraphy of the Pacific Ocean, and, of course, for all those aspects of climatic and oceanographic variability that transfer the astronomical record of varying solar insolation into quasi-cyclic sedimentological variability. For the period prior to 6 $\mathrm{Ma}$, the absolute time calibration becomes less secure, but we have 

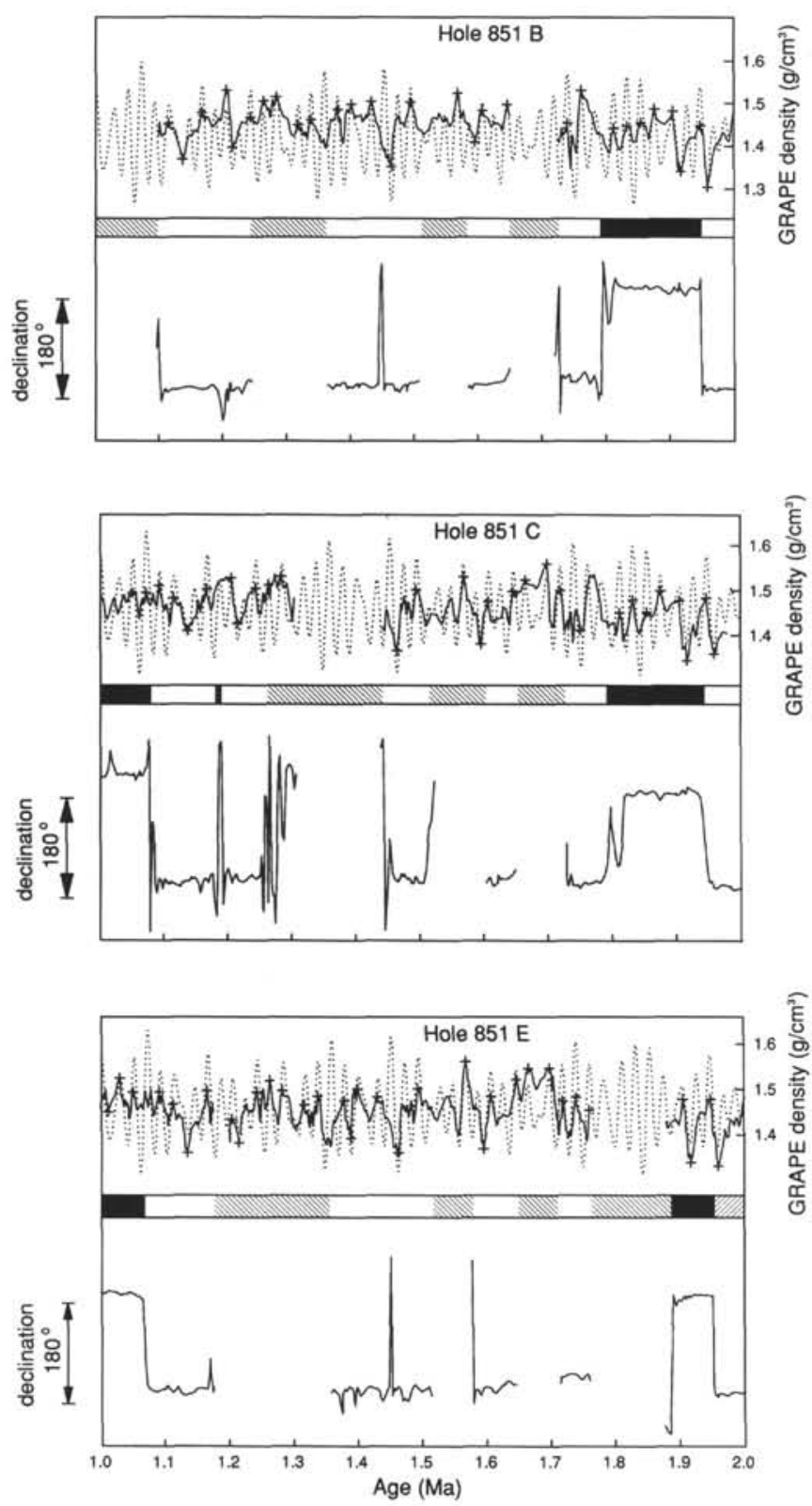

Figure 3. GRAPE density and magnetic declination for Holes 851 B, 851C, and $851 \mathrm{E}$ for the interval from 1 to $2 \mathrm{Ma}$, with orbital tuning target. Age control points are marked on the GRAPE density record. Declinations have been rotated arbitrarily for ease of comparison; the original data are shown in the site chapters in Mayer, Pisias, Janecek, et al. (1992).

defined a new magnetic polarity time scale based on astronomical tuning to the base of the Pliocene together with the anomaly distance scale given by CK92 and a new calibration by Baksi (1992) for the young side of $\mathrm{C} 5 \mathrm{n}$.

\section{ACKNOWLEDGMENTS}

We thank the crew of JOIDES Resolution for enabling the Leg 138 Shipboard Scientific Party to return with such a magnificent legacy of material and our colleagues aboard the ship for sharing nine weeks of exciting, hard work for the benefit of a generation of Pacific-loving paleoceanographers. The labor-intensive job of tuning all the GRAPE density records would have been impossible without the support of NERC through Grant GST/02/554, and we are especially grateful for their contribution to the cost of a Sun SPARCstation as soon as the need for this became apparent. We thank Fritz Hilgen and André Berger for their helpful reviews of the submitted manuscript, and Ted Moore and Jan Backman in particular for valuable discussions at several stages in its progress.

\section{REFERENCES ${ }^{*}$}

Arrhenius, G., 1952. Sediment cores from the east Pacific. Rep. Swed. DeepSea Exped. 1947-1948, 5:6-227.

Baksi, A.K., 1992. A ${ }^{40} \mathrm{Ar} /{ }^{39} \mathrm{Ar}$ age for the termination of Chron 5: a new calibration point for the Miocene section of the GPTS. Eos (Suppl.), October 27, 1992, 73:630.

Baksi, A.K., and Farrar, E., 1990. Evidence for errors in the geomagnetic polar time-scale at $17-15 \mathrm{Ma}:{ }^{40} \mathrm{Ar} /{ }^{39} \mathrm{Ar}$ dating of basalts from the Pacific Northwest. Geophys. Res. Lett., 17:1117-1120.

Baksi, A.K., Hsu, V., McWilliams, M.O., and Farrar, E., $1992 .{ }^{40} \mathrm{Ar} /{ }^{39} \mathrm{Ar}$ dating of the Brunhes-Matuyama geomagnetic field reversal. Science, 256:356357.

Berger, A., 1988. Milankovitch theory and climate. Rev. Geophys., 26:624657.

Berger, A., and Loutre, M.F., 1991. Insolation values for the climate of the last 10 million years. Quat. Sci. Rev., 10:297-317.

1992. Astronomical solutions for paleoclimate studies over the last 3 million years. Earth Planet. Sci. Lett., 111:369-382.

Berger, A., Loutre, M.F., and Laskar, J., 1992. Stability of the astronomical frequencies over the Earth's history for paleoclimate studies. Science, 255:560-566.

Berger, W.H., Leckie, R.M., Janecek, T.R., Stax, R., and Takayama, T., 1993. Neogene carbonate sedimentation on Ontong Java Plateau: highlights and open questions. In Berger, W.H., Kroenke, L.W., Mayer, L.A., et al., Proc. ODP, Sci. Results, 130: College Station, TX (Ocean Drilling Program), 711-744.

Berggren, W.A., Kent, D.V., and Flynn, J.J., 1985. Jurassic to Paleogene: Part 2. Paleogene geochronology and chronostratigraphy. In Snelling, N.J. (Ed.), The Chronology of the Geological Record. Geol. Soc. London Mem., 10:141-195.

Berggren, W.A., Kent, D.V., and Van Couvering, J.A., 1985. The Neogene: Part 2. Neogene geochronology and chronostratigraphy. In Snelling, N.J. (Ed.), The Chronology of the Geological Record. Geol. Soc. London Mem., 10:211-260.

Brüggemann, W., 1992. A minimal cost function method for optimizing the age-depth relation of deep-sea sediment cores. Paleoceanography, 7:467487.

Cande, S.C., and Kent, D.V., 1992. A new geomagnetic polarity time scale for the Late Cretaceous and Cenozoic. J. Geophys. Res., 97:13917-13951.

Farrell, J.W., and Prell, W.L., 1991. Pacific $\mathrm{CaCO}_{3}$ preservation and $\delta^{18} \mathrm{O}$ since $4 \mathrm{Ma}$ : paleoceanic and paleoclimatic implications. Paleoceanography, $6: 485-498$.

Glass, B.P., Kent, D.V., Schneider, D.A., and Tauxe, L., 1991. Ivory Coast microtektite strewn field: description and relation to the Jaramillo geomagnetic event. Earth Planet. Sci. Lett., 107:182-196.

Hagelberg, T., 1993. Variability of late Neogene eastern equatorial Pacific carbonate sedimentation and global ice volume on timescales from 10,000 years to 1 million years [Ph.D. thesis]. Oregon State Univ., Corvallis.

Hagelberg, T., Shackleton, N., Pisias, N., and Shipboard Scientific Party, 1992. Development of composite depth sections for Sites 844 through 854. In Mayer, L., Pisias, N., Janecek, T., et al., Proc. ODP, Init. Repts., 138 (Pt. 1): College Station, TX (Ocean Drilling Program), 79-85.

Hall, C.M., and Farrell, J.W., 1993. Laser ${ }^{40} \mathrm{Ar} /{ }^{39} \mathrm{Ar}$ age from Ash D of ODP Site 758: dating the Brunhes-Matuyama reversal and oxygen isotope Stage 19.1. Eos (Suppl.), 74:110.

Harland, W.B., 1978. Geochronologic scales. The Geologic Time Scale. AAPG, Studies in Geology, 6:9-32.

Harland, W.B., Armstrong, R.L., Cox, A.V., Craig, L.E., Smith, A.G., and Smith, D.G., 1990. A Geologic Time Scale 1989: Cambridge (Cambridge Univ. Press).

Hays, J.D., Imbrie, J., and Shackleton, N.J., 1976. Variations in the earth's orbit: pacemaker of the ice ages. Science, 194:1121-1132.

\footnotetext{
Abbreviations for names of organizations and publication titles in ODP reference lists follow the style given in Chemical Abstracts Service Source Index (published by American Chemical Society).
} 
Hays, J.D., Saito, T., Opdyke, N.D., and Burckle, L.R., 1969. Pliocene-Pleistocene sediments of the equatorial Pacific: their paleomagnetic, biostratigraphic, and climatic record. Geol. Soc. Am. Bull., 80:1481-1513.

Heirtzler, J.R., Dickson, G.O., Herron, E.M., Pitman, W.C., III, and Le Pichon, X., 1968. Marine magnetic anomalies, geomagnetic field reversals, and motions of the ocean floor and continents. J. Geophys. Res., 73:2119-2136.

Herbert, T.D., Tauxe, L., and Tarduno, J.A., 1992. A global data base for orbital chronometry of the Plio-Pleistocene timescale. Eos (Suppl.), 73:630.

Hilgen, F.J., 1991a. Astronomical calibration of Gauss to Matuyama sapropels in the Mediterranean and implication for the Geomagnetic Polarity Time Scale. Earth Planet. Sci. Lett., 104:226-244.

, 1991b. Extension of the astronomically calibrated (polarity) time scale to the Miocene-Pliocene boundary. Earth Planet. Sci. Lett., 107:349-368.

Imbrie, J., Hays, J.D., Martinson, D.G., McIntyre, A., Mix, A.C., Morley, J.J., Pisias, N.G., Prell, W.L., and Shackleton, N.J., 1984. The orbital theory of Pleistocene climate: support from a revised chronology of the marine delta $\delta^{18} \mathrm{O}$ record. In Berger, A., Imbrie, J., Hays, J., Kukla, G., and Saltzman, B. (Eds.), Milankovitch and Climate (Pt. 1): Dordrecht (D. Reidel), 269-305.

Imbrie, J., and Imbrie, J.Z., 1980. Modeling the climatic response to orbital variations. Science, 207:943-953.

Izett, G.A., and Obradovich, J.D., 1991. Dating of the Matuyama-Brunhes Boundary based on ${ }^{40} \mathrm{Ar}-{ }^{39} \mathrm{Ar}$ ages of the Bishop Tuff and Cerro San Luis rhyolite. Geol. Soc. Am. Abstr. Prog., 23:A106.

Keir, R.S., and Berger, W.H., 1985. Late Holocene carbonate dissolution in the Equatorial Pacific: reef growth or neoglaciation? The Carbon Cycle and Atmospheric $\mathrm{CO}_{2}$ : Natural Variations Archean to Present. Am. Geophys. Union, Geophys. Monogr., 32:208-219.

Kemp, A.E.S., and Baldauf, J.G., 1993. Vast Neogene laminated diatom mat deposits from the eastern equatorial Pacific Ocean. Nature, 362:141-144.

LaBrecque, J.L., Kent, D.V., and Cande, S.C., 1977. Revised magnetic polarity time scale for Late Cretaceous and Cenozoic time. Geology, 5:330-335.

Le, J., and Shackleton, N.J., 1992. Carbonate dissolution fluctuations in the western equatorial Pacific during the late Quaternary. Paleoceanography, 7:21-42.

Mayer, L.A., 1991. Extraction of high-resolution carbonate data for paleoclimatic reconstruction. Nature, 352:148-151.

Mayer, L., Pisias, N., Janecek, T., et al., 1992. Proc. ODP, Init. Repts., 138 (Pts. 1 and 2): College Station, TX (Ocean Drilling Program).

Mayer, L.A., Pisias, N.G., and Shipboard Scientific Party, 1992. High-resolution studies of the eastern Equatorial Pacific. Eos, 73:257-262.

McDougall, I., Brown, F.H., Cerling, T.E., and Hillhouse, J.W., 1992. A reappraisal of the geomagnetic polarity time scale to $4 \mathrm{Ma}$ using data from the Turkana Basin, East Africa. Geophys. Res. Lett., 19:2349-2352.

Neeman, B.U., in press. Orbital tuning of paleoclimatic records: a reassessment. J. Climate.

Ninkovich, D., and Shackleton, N.J., 1975. Distribution, stratigraphic position and age of ash layer "L," in the Panama Basin region. Earth Planet. Sci. Lett., 27:20-34.

Obradovich, J.D., and Izett, G.A., 1992. The geomagnetic polarity time scale (GPTS) and the astronomical time scale (ATS) now in near accord. Eos (Suppl.), 73:630.

Peterson, L.C., and Backman, J., 1990. Late Cenozoic carbonate accumulation and the history of the carbonate compensation depth in the western equatorial Indian Ocean. In Duncan, R.A., Backman, J., Peterson, L.C., et al., Proc. ODP, Sci. Results, 115: College Station, TX (Ocean Drilling Program), 467-507.

Pisias, N.G., 1983. Geologic time series from deep-sea sediments: time scales and distortion by bioturbation. Mar. Geol., 51:99-113.
Pisias, N.G., and Moore, T.C., Jr., 1981. The evolution of Pleistocene climate: a time series approach. Earth Planet. Sci. Lett., 52:450-458.

Raymo, M.E., Ruddiman, W.F., Backman, J., Clement, B.M., and Martinson, D.G., 1989. Late Pliocene variation in Northern Hemisphere ice sheets and North Atlantic deep water circulation. Paleoceanography, 4:413-446.

Ruddiman, W.F., Kidd, R.B., Thomas, E., et al., 1987. Init. Repts. DSDP, 94 (Pts. 1 and 2): Washington (U.S. Govt. Printing Office).

Ruddiman, W.F., Raymo, M.E., and McIntyre, A., 1986. Matuyama 41,000year cycles: North Atlantic and Northern Hemisphere ice sheets. Earth Planet. Sci. Lett., 80:117-129.

Shackleton, N.J., Berger, A., and Peltier, W.R., 1990. An alternative astronomical calibration of the lower Pleistocene timescale based on ODP Site 677 . Trans. R. Soc. Edinburgh, Earth Sci., 81:251-261.

Shackleton, N.J., Crowhurst, S., Pisias, N., Hagelberg, T., Schneider, D., Mix, A., and ODP Leg 138 Shipboard Scientific Party, 1992. An astronomically calibrated Pliocene time scale based on Leg 138 GRAPE density records. GEOMAR Rep., 15:260. (Abstract)

Shackleton, N.J., and Opdyke, N.D., 1973. Oxygen isotope and paleomagnetic stratigraphy of equatorial Pacific core V28-238: oxygen isotope temperatures and ice volumes on a $10^{5}$ year and $10^{6}$ year scale. Quat. Res. N.Y., 3:39-55.

Shackleton, N.J., and Shipboard Scientific Party, 1992. Sedimentation rates: toward a GRAPE density stratigraphy for Leg 138 carbonate sections. In Mayer, L., Pisias, N., Janecek, T., et al., Proc. ODP, Init. Repts., 138 (Pt. 1): College Station, TX (Ocean Drilling Program), 87-91.

Shipboard Scientific Party, 1992. Explanatory notes. In Mayer, L., Pisias, N., Janecek, T., et al., Proc. ODP, Init. Repts., 138 (Pt. 1): College Station, TX (Ocean Drilling Program), 13-42.

Spell, T.L., and McDougall, I., 1992. Revisions to the age of the BrunhesMatuyama boundary and the Pleistocene geomagnetic timescale. Geophys. Res. Lett. 19:1181-1184.

Tauxe, L., Deino, A.D., Behrensmeyer, A.K., and Potts, R., 1992. Pinning down the Brunhes/Matuyama and upper Jaramillo boundaries: a reconciliation of orbital and isotopic time scales. Earth Planet. Sci. Lett., 109:561572.

Tiedemann, R., 1992. Astronomic tuning of the high-resolution benthic records from ODP Sites 658 and 659 for the last 5 million years vs. non-linear climate dynamics. GEOMAR Rep., 15:282. (Abstract)

van Andel, T.H., Heath, G.R., and Moore, T.C., Jr., 1975. Cenozoic history and paleoceanography of the central equatorial Pacific Ocean. Mem.-Geol. Soc. Am., 143.

Vincent, E., 1981. Neogene carbonate stratigraphy of Hess Rise (central North Pacific) and paleoceanographic implications. In Thiede, J., Vallier, T.L., et al., Init. Repts. DSDP, 62: Washington (U.S. Govt. Printing Office), $571-606$.

Walter, R.C., Deino, A., Renne, P., and Tauxe, L., 1992. Refining the Plio-Pleistocene GPTS using laser fusion ${ }^{40} \mathrm{Ar} /{ }^{39} \mathrm{Ar}$ tephrochronology: Case studies from the East African Rift. Eos (Suppl.), 73:629. (Abstract)

Walter, R.C., Manega, P.C., Hay, R.L., Drake, R.E., and Curtis, G.H. 1991. Laser-fusion ${ }^{40} \mathrm{Ar}{ }^{39} \mathrm{Ar}$ dating of Bed 1, Olduvai Gorge, Tanzania. Nature, 354:145-149.

Wilson, D.S., 1993. Confirmation of the astronomical calibration of the magnetic polarity timescale from sea-floor spreading. Nature, 364:788-790.

Date of initial receipt: 5 February 1993

Date of acceptance: 27 July 1993

Ms 138SR-106 
Table 17. Ages for magnetic anomalies between C3An.1n ( $t$ ) and C5Bn.1n (t), derived by re-calibrating the distances in CK92 Table 2 with C3An.1n (t) at 5.875 Ma and C5n.1n (t) at 9.639MAMa Ma.

\begin{tabular}{|c|c|c|c|}
\hline $\begin{array}{l}\text { Anomaly } \\
\text { study }\end{array}$ & $\begin{array}{c}\text { Berggren } \\
\text { et al. } \\
\text { (1985) }\end{array}$ & CK92 & $\begin{array}{l}\text { This } \\
\text { study }\end{array}$ \\
\hline C3An.In (t) & 5.35 & 5.705 & 5.875 \\
\hline C3An. $\ln (0)$ & 5.53 & 5.946 & 6.122 \\
\hline C $3 A n \cdot 2 n(t)$ & 5.68 & 6.078 & 6.256 \\
\hline C $3 A n \cdot 2 n(0)$ & 5.89 & 6.376 & 6.555 \\
\hline $\mathrm{C} 3 \mathrm{Bn}(\mathrm{t})$ & 6.37 & 6.744 & 6.919 \\
\hline $\mathrm{C} 3 \mathrm{Bn}(\mathrm{o})$ & 6.50 & 6.901 & 7.072 \\
\hline $\mathrm{C} 4 \mathrm{n} . \ln (\mathrm{t})$ & 6.70 & 7.245 & 7.406 \\
\hline$C 4 n \cdot \ln (0)$ & 6.78 & 7.376 & 7.533 \\
\hline$C 4 n .2 n(t)$ & 6.85 & 7.464 & 7.618 \\
\hline$C 4 n \cdot 2 n(0)$ & 7.28 & 7.892 & 8.027 \\
\hline$C 4 r \cdot \ln (t)$ & 7.35 & 8.047 & 8.174 \\
\hline C4r.In (o) & 7.41 & 8.079 & 8.205 \\
\hline $\mathrm{C} 4 \mathrm{An}(\mathrm{t})$ & 7.90 & 8.529 & 8.631 \\
\hline C4An (o) & 8.21 & 8.861 & 8.945 \\
\hline $\mathrm{C} 4 \mathrm{Ar} \cdot \ln (\mathrm{t})$ & 8.41 & 9.069 & 9.142 \\
\hline C4Ar. In (o) & 8.50 & 9.149 & 9.218 \\
\hline C4Ar.2n ( & 8.71 & 9.428 & 9.482 \\
\hline C4Ar.2n (o) & 8.80 & 9.491 & 9.543 \\
\hline$C 5 n .1 n(t)$ & 8.92 & 9.5 & 9.639 \\
\hline C5n.1n (o) & N.D. & 9.735 & 9.775 \\
\hline C5n.2n (t) & N.D. & 9.777 & 9.815 \\
\hline C $5 n \cdot 2 n(0)$ & 10.42 & 10.834 & 10.839 \\
\hline C5r.In (t) & 10.54 & 10.940 & 10.943 \\
\hline C $5 r . \ln (0)$ & 10.59 & 10.989 & 10.991 \\
\hline C5r.2n (t) & 11.03 & 11.378 & 11.373 \\
\hline C.5r.2n (o) & 11.09 & 11.434 & 11.428 \\
\hline C5An. $\ln$ & 11.55 & 11.852 & 11.841 \\
\hline C5An. In (o) & 11.73 & 12.000 & 11.988 \\
\hline C5An.2n (t) & 11.86 & 12.108 & 12.096 \\
\hline C5An.2n (o) & 12.12 & 12.333 & 12.320 \\
\hline C5Ar. In (t) & 12.46 & 12.618 & 12.605 \\
\hline C5Ar.1n (o) & 12.49 & 12.649 & 12.637 \\
\hline C5Ar.2n (t) & 12.58 & 12.718 & 12.705 \\
\hline C 5 Ar.2n (o) & 12.62 & 12.764 & 12.752 \\
\hline C5AAn (t) & 12.83 & 12.941 & 12.929 \\
\hline C5AAn (o) & 13.01 & 13.094 & 13.083 \\
\hline $\mathrm{C} 5 \mathrm{ABn}(\mathrm{t})$ & 13.20 & 12.263 & 13.252 \\
\hline C5ABn (o) & 13.46 & 13.476 & 13.466 \\
\hline $\operatorname{CsACn}(t)$ & 13.69 & 13.674 & 13.666 \\
\hline C5ACn (o) & 14.08 & 14.059 & 14.053 \\
\hline C5ADn (t) & 14.20 & 14.164 & 14.159 \\
\hline C5ADn (o) & 14.66 & 14.608 & 14.607 \\
\hline $\mathrm{C} 5 \mathrm{Bn} \cdot \ln (\mathrm{t})$ & 14.87 & 14.800 & 14.800 \\
\hline
\end{tabular}

Note: N.D. = not determined.
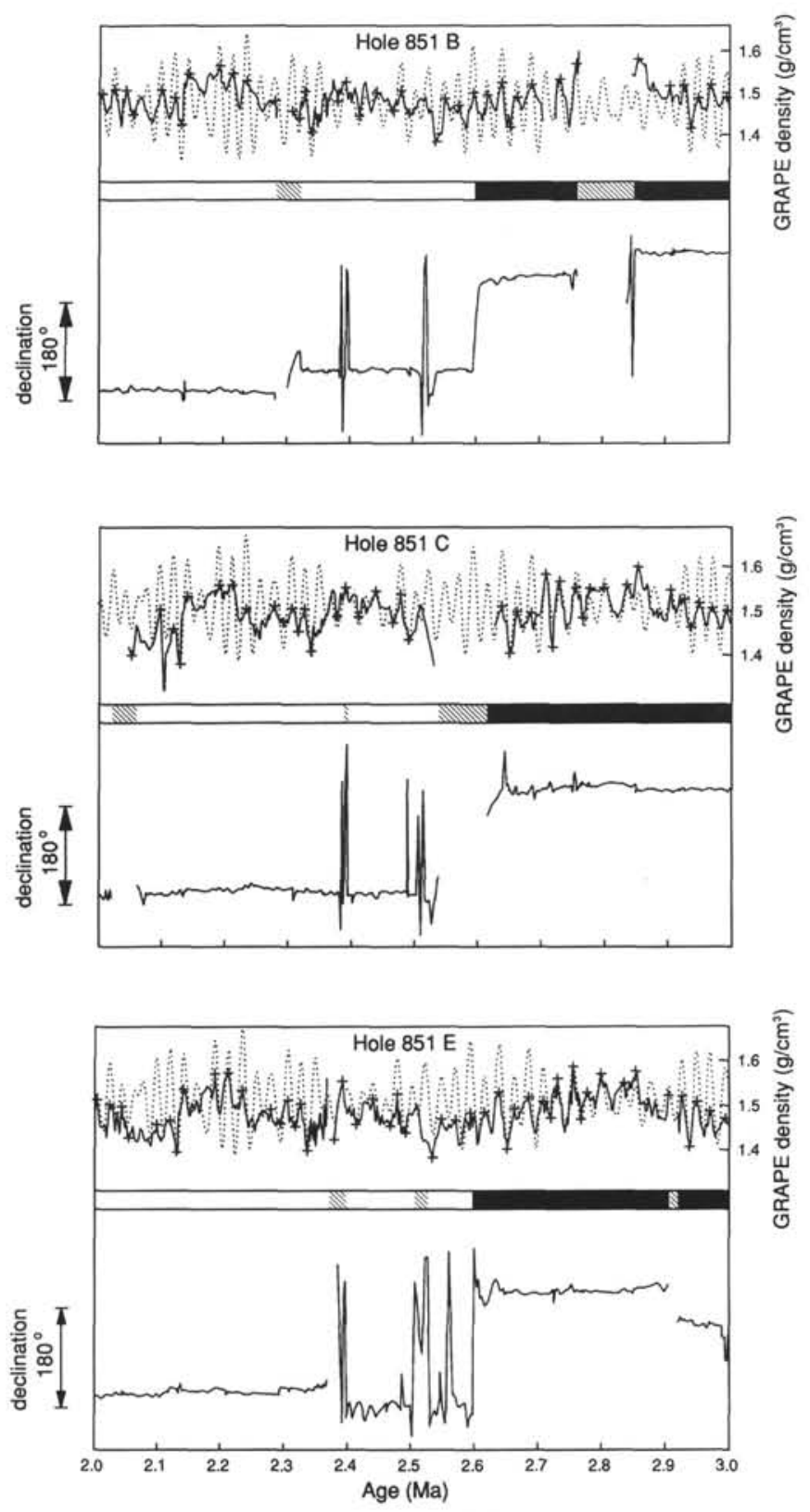

Figure 4. GRAPE density and magnetic declination for Holes 851B, 851C, and $851 \mathrm{E}$ for the interval from 2 to $3 \mathrm{Ma}$, with orbital tuning target. Age control points are marked on the GRAPE density record. Declinations have been rotated arbitrarily for ease of comparison; the original data are shown in the site chapters in Mayer, Pisias, Janecek, et al. (1992). 
Table 18. Accumulation rates (mcd scale) estimated in overlapping $\mathbf{0 . 2}$ m.y. intervals for Site $\mathbf{8 4 4}$ (from Table 1).

\begin{tabular}{|c|c|c|c|c|c|}
\hline $\begin{array}{l}\text { Age } \\
\text { (Ma) }\end{array}$ & $\begin{array}{l}\text { Depth } \\
\text { (mcd) }\end{array}$ & $\begin{array}{c}\text { Rate } \\
(\mathrm{m} / \mathrm{m} . \mathrm{y} .)\end{array}$ & $\begin{array}{l}\text { Age } \\
\text { (Ma) }\end{array}$ & $\begin{array}{l}\text { Depth } \\
\text { (mcd) }\end{array}$ & $\begin{array}{c}\text { Rate } \\
(\mathrm{m} / \mathrm{m} . \mathrm{y} .)\end{array}$ \\
\hline 0.0 & 0.00 & & 8.5 & 52.90 & 6.2 \\
\hline 0.1 & 1.53 & 12.9 & 8.6 & 53.53 & 7.4 \\
\hline 0.2 & 2.58 & 11.5 & 8.8 & 55.35 & 9.6 \\
\hline 0.3 & 3.82 & 12.8 & 8.9 & 56.32 & 8.7 \\
\hline 0.4 & 5.14 & 14.3 & 9.0 & 57.10 & 7.1 \\
\hline 0.5 & 6.68 & 15.1 & 9.1 & 57.73 & 7.9 \\
\hline 0.6 & 8.16 & 12.4 & 9,2 & 58.69 & 8.5 \\
\hline 0.7 & 9.16 & 10.4 & 9.3 & 59.43 & 6.9 \\
\hline 0.8 & 10.23 & 9.6 & 9.4 & 60.07 & 7.9 \\
\hline 0.9 & 11.07 & 8.5 & 9.5 & 61.00 & 10.7 \\
\hline 1.0 & 11.93 & 9.0 & 9.6 & 62.22 & 10.8 \\
\hline 1.1 & 12.86 & 8.6 & 9.7 & 63.15 & 10.9 \\
\hline 1.2 & 13.65 & 7.8 & 9.8 & 64.39 & 12.3 \\
\hline 1.3 & 14.43 & 7.8 & 9.9 & 65.62 & 12.3 \\
\hline 1.4 & 15.21 & 7.8 & 10.0 & 66.86 & 11.9 \\
\hline 1.5 & 15.99 & 7.8 & 10.1 & 68.00 & 11.3 \\
\hline 1.6 & 16.77 & 7.8 & 10.2 & 69.12 & 11.2 \\
\hline 1.7 & 17.55 & 7.5 & 10.3 & 70.24 & 11.2 \\
\hline 1.9 & 18.86 & 5.5 & 10.4 & 71.36 & 11.2 \\
\hline 2.0 & 19.38 & 5.0 & 10.5 & 72.47 & 12.3 \\
\hline 2.1 & 19.85 & 4.7 & 10.6 & 73.82 & 14.7 \\
\hline 2.2 & 20.32 & 4.7 & 10.7 & 75.42 & 18.9 \\
\hline 2.3 & 20.79 & 4.7 & 10.8 & 77.59 & 21.7 \\
\hline 2.4 & 21.26 & 4.7 & 10.9 & 79.76 & 22.1 \\
\hline 2.5 & 21.73 & 4.7 & 11.0 & 82.02 & 26.9 \\
\hline 2.6 & 22.20 & 4.1 & 11.1 & 85.14 & 31.2 \\
\hline 2.7 & 22.54 & 3.4 & 11.2 & 88.26 & 31.2 \\
\hline 2.8 & 22.88 & 3.4 & 11.3 & 91.37 & 32.0 \\
\hline 2.9 & 23.23 & 3.4 & 11.4 & 94.67 & 35.3 \\
\hline 3.0 & 23.57 & 3.2 & 11.5 & 98.42 & 37.6 \\
\hline 3.1 & 23.87 & 3.6 & 11.6 & 102.18 & 37.6 \\
\hline 3.2 & 24.28 & 3.0 & 11.7 & 105.94 & 37.6 \\
\hline 3.3 & 24.47 & 2.2 & 11.8 & 109.70 & 37.6 \\
\hline 3.4 & 24.72 & 3.0 & 11.9 & 113.46 & 37.0 \\
\hline 3.5 & 25.07 & 3.5 & 12.0 & 117.10 & 32.0 \\
\hline 3.6 & 25.41 & 3.1 & 12.1 & 119.87 & 27.6 \\
\hline 3.7 & 25.68 & 2.6 & 12.2 & 122.63 & 27.6 \\
\hline 3.8 & 25.94 & 2.6 & 12.3 & 125.39 & 27.6 \\
\hline 3.9 & 26.20 & 2.6 & 12.4 & 128.16 & 27.6 \\
\hline 4.0 & 26.45 & 2.6 & 12.5 & 130.92 & 27.6 \\
\hline 4.1 & 26.71 & 2.6 & 12.6 & 133.68 & 33.4 \\
\hline 4.2 & 26.98 & 3.1 & 12.7 & 137.60 & 42.4 \\
\hline 4.3 & 27.32 & 3.4 & 12.8 & 142.16 & 45.6 \\
\hline 4.4 & 27.65 & 3.3 & 12,9 & 146.72 & 43.1 \\
\hline 4.5 & 27.99 & 3.7 & 13.0 & 150.77 & 39.5 \\
\hline 4.6 & 28.38 & 3.8 & 13.1 & 154.63 & 38.5 \\
\hline 4.7 & 28.75 & 3.6 & 13.2 & 158.48 & 37.8 \\
\hline 4.8 & 29.10 & 3.6 & 13.3 & 162.18 & 36.2 \\
\hline 4.9 & 29.47 & 4.5 & 13.4 & 165.72 & 35.4 \\
\hline 5.0 & 30.01 & 5.5 & 13.5 & 169.26 & 35.4 \\
\hline 5.1 & 30.56 & 5.6 & 13.6 & 172.80 & 35.4 \\
\hline 5.2 & 31.12 & 5.7 & 13.7 & 176.35 & 35.4 \\
\hline 5.3 & 31.70 & 5.9 & 13.8 & 179.89 & 35.4 \\
\hline 5.4 & 32.29 & 5.9 & 13.9 & 183.43 & 35.4 \\
\hline 5.5 & 32.88 & 5.9 & 14.0 & 186.97 & 35.3 \\
\hline 5.6 & 33.47 & 5.9 & 14.1 & 190.49 & 34.9 \\
\hline 5.7 & 34.07 & 5.9 & 14.2 & 193.96 & 34.7 \\
\hline 5.8 & 34.66 & 6.1 & 14.3 & 197.43 & 34.7 \\
\hline 5.9 & 35.28 & 6.6 & 14.4 & 200.90 & 14.7 \\
\hline 6.0 & 35.99 & 7.1 & 14.5 & 204.37 & 34.7 \\
\hline 6.1 & 36.69 & 7.2 & 14.6 & 207.84 & 34.7 \\
\hline 6.2 & 37.43 & 6.4 & 14.7 & 211.31 & 34.7 \\
\hline 6.3 & 37.98 & 4.1 & 14.8 & 214.78 & 34.7 \\
\hline 6.4 & 38.26 & 2.9 & 14.9 & 218.25 & 36.8 \\
\hline 6.5 & 38.55 & 3.6 & 15.0 & 222.14 & 41.1 \\
\hline 6.6 & 38.99 & 5.4 & 15.1 & 226.47 & 43.3 \\
\hline 6.7 & 39.62 & 6.3 & 15.2 & 230.80 & 43.3 \\
\hline 6.8 & 40.25 & 6.3 & 15.3 & 235.12 & 43.3 \\
\hline 6.9 & 40.88 & 5.9 & 15.4 & 239.45 & 43.3 \\
\hline 7.0 & 41.42 & 5.2 & 15.5 & 243.77 & 43.3 \\
\hline 7.1 & 41.91 & 4.5 & 15.7 & 252.43 & 43.3 \\
\hline 7.2 & 42.32 & 4.0 & 15.8 & 256.75 & 42.4 \\
\hline 7.3 & 42.72 & 4.0 & 15.9 & 260.91 & 41.2 \\
\hline 7.4 & 43.13 & 6.8 & 16.0 & 265.00 & 40.9 \\
\hline 7.5 & 44.08 & 8.5 & 16.1 & 269.08 & 40.9 \\
\hline 7.6 & 44.83 & 9.4 & 16.2 & 273.17 & 40.9 \\
\hline 7.7 & 45.95 & 11.7 & 16.3 & 277.25 & 40.9 \\
\hline 7.8 & 47.17 & 12.2 & 16.4 & 281.34 & 40.9 \\
\hline 7.9 & 48.40 & 12.2 & 16.5 & 285.42 & 40.9 \\
\hline 8.0 & 49.62 & 10.0 & 16.6 & 289.51 & 40.9 \\
\hline 8.1 & 50.41 & 7.0 & 16.7 & 293.59 & 40.9 \\
\hline 8.2 & 51.03 & 6.2 & 16.8 & 297.68 & 40.9 \\
\hline 8.3 & 51.65 & 6.2 & 16.9 & 301.76 & 40.9 \\
\hline 8.4 & 52.28 & 6.2 & 17.0 & 305.85 & \\
\hline
\end{tabular}

Table 19. Accumulation rates (mcd scale) estimated in overlapping $\mathbf{0 . 2}$ m.y. intervals for Site $\mathbf{8 4 5}$ (from Table 2).

\begin{tabular}{|c|c|c|c|c|c|}
\hline $\begin{array}{l}\text { Age } \\
\mathrm{Ma}\end{array}$ & $\begin{array}{l}\text { Depth } \\
\text { (med) }\end{array}$ & $\begin{array}{c}\text { Rate } \\
\mathrm{m} / \mathrm{m} . \mathrm{y} .\end{array}$ & $\begin{array}{l}\text { Age } \\
\mathrm{Ma}\end{array}$ & $\begin{array}{l}\text { Depth } \\
\text { (mcd) }\end{array}$ & $\begin{array}{c}\text { Rate } \\
\mathrm{m} / \mathrm{m} . \mathrm{y} .\end{array}$ \\
\hline 0.0 & 0.00 & & 0.0 & 0.00 & \\
\hline 0.1 & 3.00 & 30.0 & 8.4 & 124.89 & 20.9 \\
\hline 0.2 & 6.01 & 30.0 & 8.5 & 126.98 & 20.9 \\
\hline 0.3 & 9.01 & 30.0 & 8.6 & 129.06 & 21.4 \\
\hline 0.4 & 12.02 & 25.4 & 8.7 & 131.25 & 22.2 \\
\hline 0.5 & 14.10 & 20.3 & 8.8 & 133.49 & 22.4 \\
\hline 0.6 & 16.08 & 19.8 & 8.9 & 135.73 & 20.8 \\
\hline 0.7 & 18.06 & 19.8 & 9.0 & 137.65 & 17.9 \\
\hline 0.8 & 20.04 & 19.8 & 9.1 & 139.31 & 17.2 \\
\hline 0.9 & 22.02 & 19.8 & 9.2 & 141.09 & 16.3 \\
\hline 1.0 & 24.00 & 19.8 & 9.3 & 142.56 & 14.3 \\
\hline 1.1 & 25.98 & 19.8 & 9.4 & 143.95 & 15.0 \\
\hline 1.2 & 27.96 & 19.8 & 9.5 & 145.56 & 16.9 \\
\hline 1.3 & 29.94 & 19.8 & 9.6 & 147.32 & 16.7 \\
\hline 1.4 & 31.92 & 19.8 & 9.7 & 148.91 & 16.5 \\
\hline 1.5 & 33.90 & 19.8 & 9.8 & 150.61 & 15.2 \\
\hline 1.6 & 35.88 & 19.8 & 9.9 & 151.96 & 13.6 \\
\hline 1.7 & 37.86 & 19.8 & 10.0 & 153.34 & 13.8 \\
\hline 1.8 & 39.84 & 19.8 & 10.1 & 154.72 & 13.8 \\
\hline 1.9 & 41.82 & 17.9 & 10.2 & 156.10 & 13.8 \\
\hline 2.0 & 43,42 & 12.9 & 10.3 & 157.49 & 13.8 \\
\hline 2.1 & 44.41 & 9.9 & 10.4 & 158.87 & 13.8 \\
\hline 2.2 & 45.40 & 9.9 & 10.5 & 160.25 & 13.8 \\
\hline 2.3 & 46.40 & 9.9 & 10.6 & 161.64 & 13.8 \\
\hline 2.4 & 47.39 & 9.9 & 10.7 & 163.02 & 13.8 \\
\hline 2.5 & 48.39 & 9.9 & 10.8 & 164.40 & 12.8 \\
\hline 2.6 & 49.38 & 9.9 & 10.9 & 165.58 & 12.8 \\
\hline 2.7 & 50.37 & 9.9 & 11.0 & 166.95 & 16.5 \\
\hline 2.8 & 51.37 & 9.9 & 11.1 & 168.89 & 19.3 \\
\hline 2.9 & 52.36 & 9.9 & 11.2 & 170.82 & 19.3 \\
\hline 3.0 & 53.35 & 10.9 & 11.3 & 172.76 & 20.7 \\
\hline 3.1 & 54.54 & 11.8 & 11.4 & 174.96 & 22.0 \\
\hline 3.2 & 55.72 & 11.9 & 11.5 & 177.15 & 20.5 \\
\hline 3.3 & 56.92 & 11.2 & 11.6 & 179.06 & 19.1 \\
\hline 3.4 & 57.95 & 9.7 & 11.7 & 180.96 & 19.1 \\
\hline 3.5 & 58.87 & 9.1 & 11.8 & 182.87 & 20.3 \\
\hline 3.6 & 59.78 & 9.4 & 11.9 & 185.02 & 22.7 \\
\hline 3.7 & 60.75 & 9.7 & 12.0 & 187.41 & 26.4 \\
\hline 3.8 & 61.73 & 9.8 & 12.1 & 190.30 & 28.9 \\
\hline 3.9 & 62.71 & 9.8 & 12.2 & 193.19 & 28.9 \\
\hline 4.0 & 63.68 & 9.8 & 12.3 & 196.09 & 28.9 \\
\hline 4.1 & 64.66 & 9.7 & 12.4 & 198.98 & 28.9 \\
\hline 4.2 & 65.63 & 8.9 & 12.5 & 201.87 & 28.9 \\
\hline 4.3 & 66.44 & 9.7 & 12.6 & 204.77 & 30.6 \\
\hline 4.4 & 67.57 & 11.4 & 12.7 & 207.99 & 32.1 \\
\hline 4.5 & 68.73 & 10.8 & 12.8 & 211.19 & 32.8 \\
\hline 4.6 & 69.72 & 10.0 & 12.9 & 214.56 & 32.2 \\
\hline 4.7 & 70.74 & 9.7 & 13.0 & 217.63 & 30.6 \\
\hline 4.8 & 71.67 & 7.9 & 13.1 & 220.67 & 33.0 \\
\hline 4.9 & 72.31 & 9.1 & 13.2 & 224.22 & 31.6 \\
\hline 5.0 & 73.49 & 11.3 & 13.3 & 226.99 & 23.3 \\
\hline 5.1 & 74.56 & 10.7 & 13.4 & 228.89 & 19.1 \\
\hline 5.2 & 75.63 & 13.1 & 13.5 & 230.80 & 19.1 \\
\hline 5.3 & 77.19 & 16.8 & 13.6 & 232.71 & 19.1 \\
\hline 5.4 & 78.98 & 17.9 & 13.7 & 234.61 & 19.1 \\
\hline 5.5 & 80.77 & 17.9 & 13.8 & 236.52 & 19.1 \\
\hline 5.6 & 82.56 & 17.9 & 13.9 & 238.43 & 19.1 \\
\hline 5.7 & 84.35 & 17.9 & 14.0 & 240.33 & 19.1 \\
\hline 5.8 & 86.15 & 17.1 & 14.1 & 242.24 & 19.1 \\
\hline 5.9 & 87.77 & 13.6 & 14.2 & 244.15 & 19.1 \\
\hline 6.0 & 88.87 & 11.1 & 14.3 & 246.05 & 19.1 \\
\hline 6.1 & 89.98 & 14.8 & 14.4 & 247.96 & 19.1 \\
\hline 6.2 & 91.84 & 17.6 & 14.5 & 249.87 & 19.1 \\
\hline 6.3 & 93.49 & 13.8 & 14.6 & 251.78 & 19.1 \\
\hline 6.4 & 94.59 & 11.1 & 14.7 & 253.68 & 19.1 \\
\hline 6.5 & 95.70 & 11.8 & 14.8 & 255.59 & 19.1 \\
\hline 6.6 & 96.94 & 13.3 & 14.9 & 257.50 & 19.1 \\
\hline 6.7 & 98.35 & 14.1 & 15.0 & 259.40 & 19.1 \\
\hline 6.8 & 99.76 & 14.1 & 15.1 & 261.31 & 19.1 \\
\hline 6.9 & 101.17 & 13.0 & 15.2 & 263.22 & 19.1 \\
\hline 7.0 & 102.36 & 12.1 & 15.3 & 265.12 & 19.1 \\
\hline 7.1 & 103.59 & 13.4 & 15.4 & 267.03 & 19.1 \\
\hline 7.2 & 105.04 & 14.6 & 15.5 & 268.94 & 19.1 \\
\hline 7.3 & 106.50 & 14.6 & 15.6 & 270.84 & 19.1 \\
\hline 7.4 & 107.95 & 15.4 & 15.7 & 272.75 & 19.1 \\
\hline 7.5 & 109.58 & 14.4 & 15.8 & 274.66 & 31.5 \\
\hline 7.6 & 110.83 & 15.5 & 15.9 & 279.05 & 49.3 \\
\hline 7.7 & 112.68 & 19.4 & 16.0 & 284.52 & 54.6 \\
\hline 7.8 & 114.70 & 20.2 & 16.1 & 289.98 & 54.6 \\
\hline 7.9 & 116.72 & 20.2 & 16.2 & 295.44 & 54.6 \\
\hline 8.0 & 118.74 & 15.9 & 16.3 & 300.91 & 54.6 \\
\hline 8.1 & 119.91 & 10.1 & 16.4 & 306.37 & \\
\hline 8.2 & 120.77 & 14.5 & & & \\
\hline
\end{tabular}




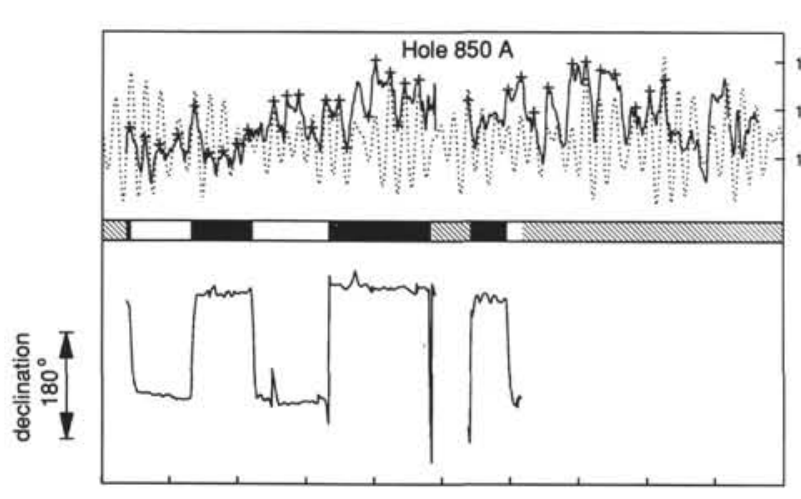

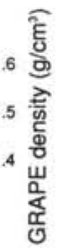

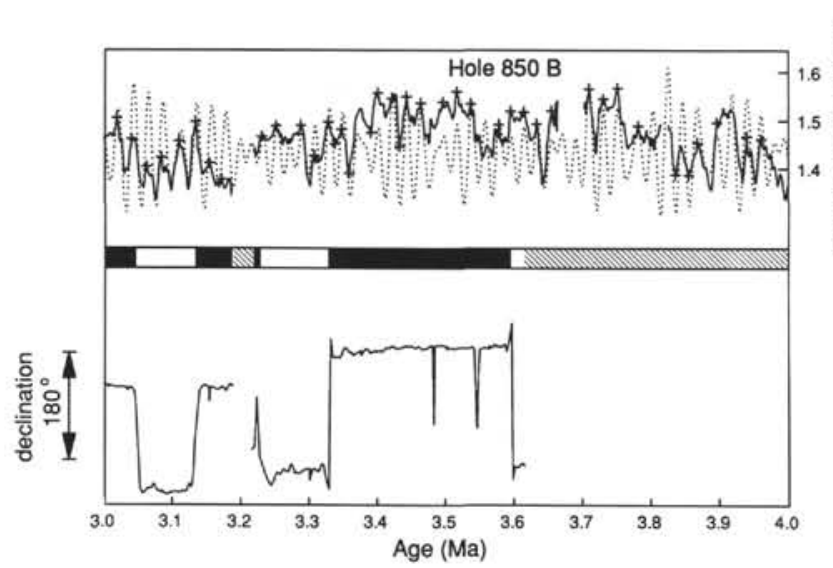

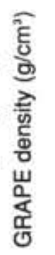

Figure 5. GRAPE density and magnetic declination for Holes $850 \mathrm{~A}$ and $850 \mathrm{~B}$ for the interval from 3 to $4 \mathrm{Ma}$, with orbital tuning target. Age control points are marked on the GRAPE density record. Declinations have been rotated arbitrarily for ease of comparison; the original data are shown in the site chapters in Mayer, Pisias, Janecek, et al. (1992).
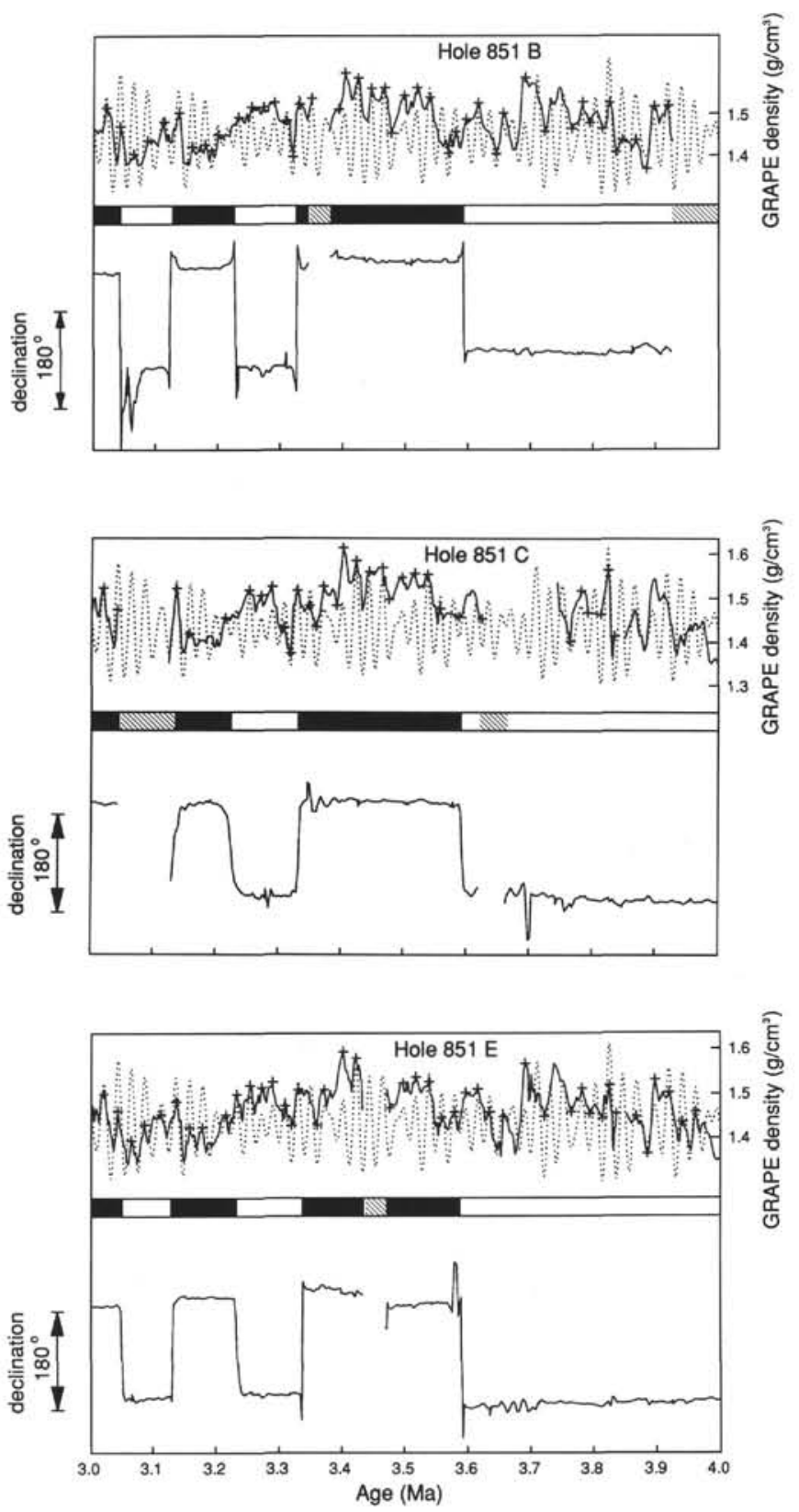

Figure 6. GRAPE density and magnetic declination for Holes 851B, 851C, and $851 \mathrm{E}$ for the interval from 3 to $4 \mathrm{Ma}$, with orbital tuning target. Age control points are marked on the GRAPE density record. Declinations have been rotated arbitrarily for ease of comparison; the original data are shown in the site chapters in Mayer, Pisias, Janecek, et al. (1992). 

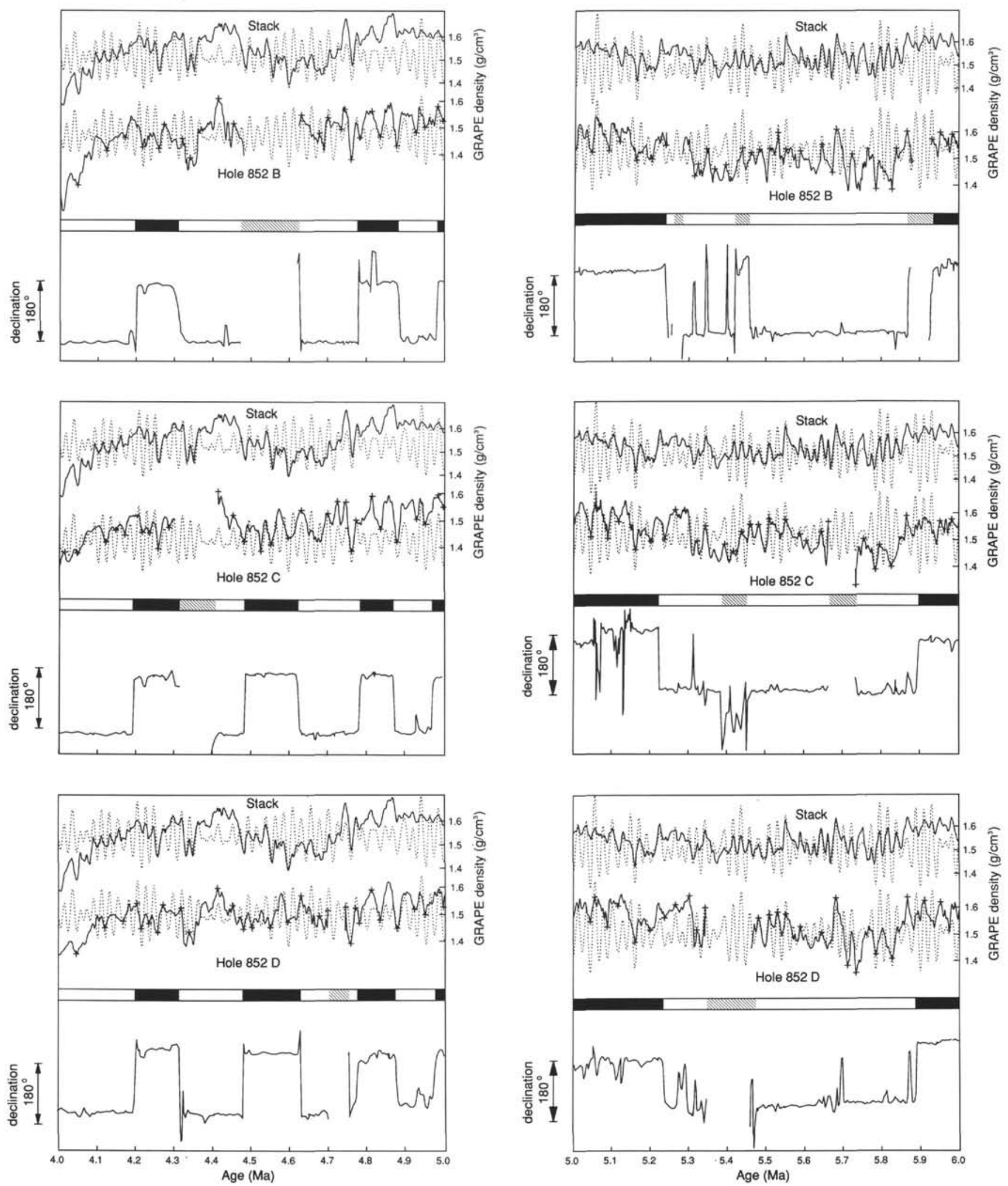

Figure 7. GRAPE density (middle) and magnetic declination (below) for Holes $852 \mathrm{~B}, 852 \mathrm{C}$, and $852 \mathrm{D}$ for the interval from 4 to $5 \mathrm{Ma}$, with orbital tuning target. Age control points are marked on the GRAPE density record. Above: stacked GRAPE density records of Sites 849,850 , and 851 for the same interval. Declinations have been rotated arbitrarily for ease of comparison; the original data are shown in the site chapters in Mayer, Pisias, Janecek, et al. (1992).

Figure 8. GRAPE density (middle) and magnetic declination (below) for Holes $852 \mathrm{~B}, 852 \mathrm{C}$, and $852 \mathrm{D}$ for the interval from 5 to $6 \mathrm{Ma}$, with orbital tuning target. Age control points are marked on the GRAPE density record. Above: stacked GRAPE density records of Sites 849,850 , and 851 for the same interval. Declinations have been rotated arbitrarily for ease of comparison; the original data are shown in the site chapters in Mayer, Pisias, Janecek, et al. (1992). 

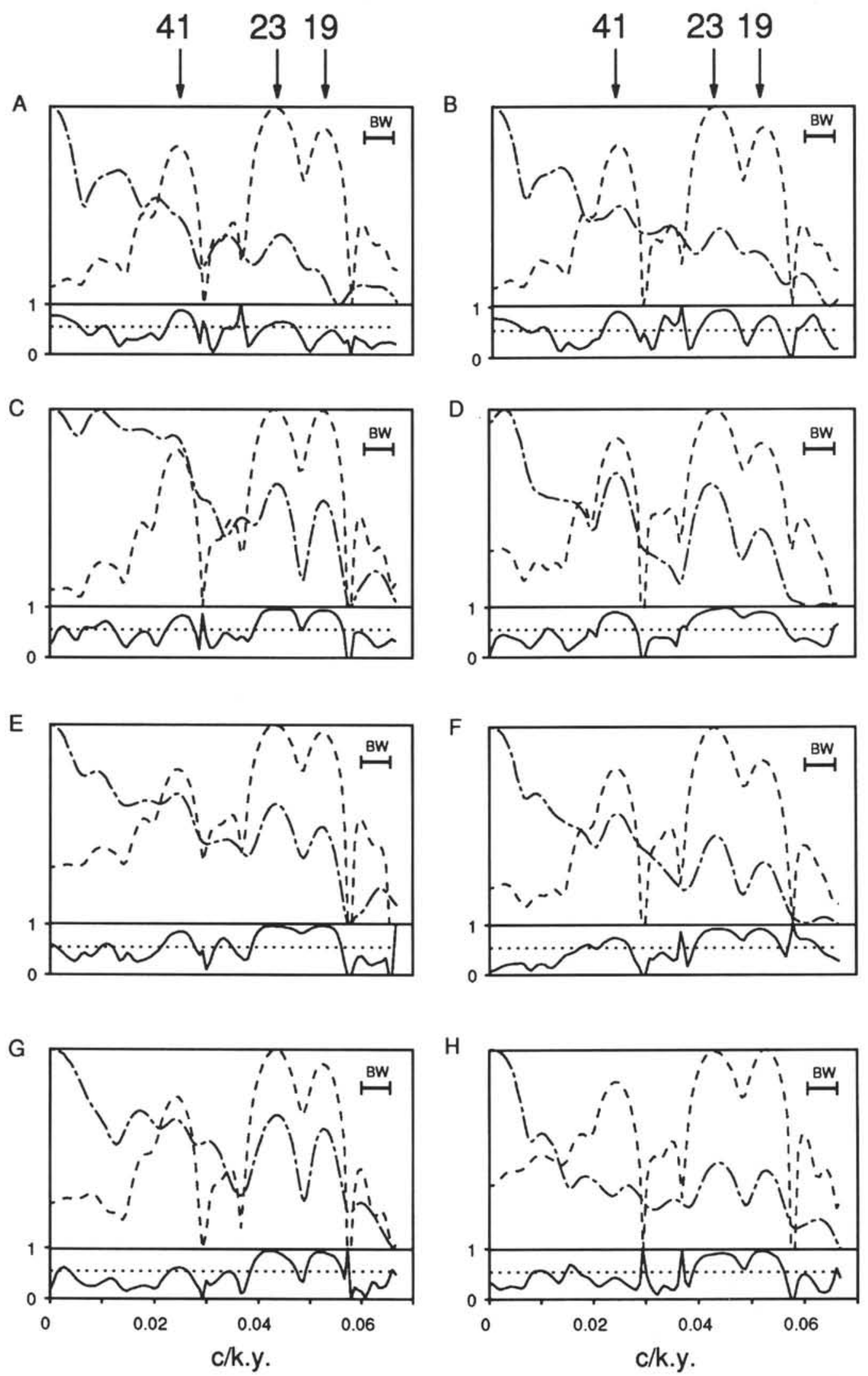

Figure 9. Cross-spectral analysis of average GRAPE density for Sites 849,850 , and 851 vs. summer insolation at $65^{\circ} \mathrm{N}$. A. 0 to $1 \mathrm{Ma}$ (from Tables 6, 7, and 8). B. 0 to $1 \mathrm{Ma}$ (from Table 12). C. 1 to $2 \mathrm{Ma}$. D. 2 to 3 Ma. E. 3 to 4 Ma. F. 4 to 5 Ma. G. 5 to 6 Ma. H. 6 to 7 Ma. The time series were sampled at 3-k.y. intervals and cross-spectra calculated for 80 lags. Dashed line $=$ insolation spectra; dash-dotted line $=$ GRAPE spectra; solid line $=$ coherency; dotted line $=80 \%$ confidence limit for coherency. Arrows at the top of the figure identify prominent peaks in insolation variance associated with obliquity (41 k.y.) and precession (23 and 19 k.y.). 
Table 20. Accumulation rates (mcd scale) estimated in overlapping 0.2 m.y. intervals for Site 846 (from Table 3).

\begin{tabular}{|c|c|c|c|c|c|c|c|c|}
\hline $\begin{array}{l}\text { Age } \\
\text { (Ma) }\end{array}$ & $\begin{array}{l}\text { Depth } \\
\text { (mcd) }\end{array}$ & $\begin{array}{c}\text { Rate } \\
(\mathrm{m} / \mathrm{m} . \mathrm{y} .)\end{array}$ & $\begin{array}{l}\text { Age } \\
\text { (Ma) }\end{array}$ & $\begin{array}{l}\text { Depth } \\
\text { (mcd) }\end{array}$ & $\begin{array}{c}\text { Rate } \\
\text { (m/m.y.) }\end{array}$ & $\begin{array}{l}\text { Age } \\
\text { (Ma) }\end{array}$ & $\begin{array}{l}\text { Depth } \\
\text { (mcd) }\end{array}$ & $\begin{array}{c}\text { Rate } \\
(\mathrm{m} / \mathrm{m} . \mathrm{y} .)\end{array}$ \\
\hline 0.0 & 0.00 & & 6.1 & 249.80 & 49.2 & 12.2 & 374.73 & 18.0 \\
\hline 0.1 & 4.39 & 41.5 & 6.2 & 253.20 & 29.6 & 12.3 & 376.53 & 18.0 \\
\hline 0.2 & 8.30 & 35.8 & 6.3 & 255.73 & 47.5 & 12.4 & 378.33 & 18.0 \\
\hline 0.3 & 11.55 & 36.0 & 6.4 & 262.71 & 48.0 & 12.5 & 380.13 & 18.0 \\
\hline 0.4 & 15.51 & 40.1 & 6.5 & 265.33 & 27.7 & 12.6 & 381.92 & 18.0 \\
\hline 0.5 & 19.56 & 35.4 & 6.6 & 268.24 & 27.2 & 12.7 & 383.72 & 18.0 \\
\hline 0.6 & 22.58 & 31.6 & 6.7 & 270.76 & 25.2 & 12.8 & 385.52 & 18.0 \\
\hline 0.7 & 25.89 & 34.5 & 6.8 & 273.28 & 25.2 & 12.9 & 387.31 & 18.0 \\
\hline 0.8 & 29.47 & 40.4 & 6.9 & 275.80 & 25.2 & 13.0 & 389.11 & 18.0 \\
\hline 0.9 & 33.98 & 35.6 & 7.0 & 278.32 & 29.4 & 13.1 & 390.91 & 18.0 \\
\hline 1.0 & 36.59 & 30.9 & 7.1 & 281.69 & 42.2 & 13.2 & 392.71 & 18.0 \\
\hline 1.1 & 40.16 & 38.0 & 7.2 & 286.77 & 39.9 & 13.3 & 394.50 & 23.4 \\
\hline 1.2 & 44.19 & 32.5 & 7.3 & 289.67 & 37.8 & 13.4 & 397.39 & 28.5 \\
\hline 1.3 & 46.67 & 32.2 & 7.4 & 294.32 & 38.7 & 13.5 & 400.20 & 19.1 \\
\hline 1.4 & 50.64 & 36.3 & 7.5 & 297.41 & 24.7 & 13.6 & 401.21 & 10.1 \\
\hline 1.5 & 53.93 & 31.9 & 7.6 & 299.26 & 18.4 & 13.7 & 402.22 & 10.1 \\
\hline 1.6 & 57.02 & 36.2 & 7.7 & 301.10 & 19.4 & 13.8 & 403.23 & 10.1 \\
\hline 1.7 & 61.17 & 38.4 & 7.8 & 303.14 & 21.0 & 13.9 & 404.24 & 10.1 \\
\hline 1.8 & 64.69 & 38.9 & 7.9 & 305.30 & 20.7 & 14.0 & 405.26 & 10.1 \\
\hline 1.9 & 68.96 & 47.0 & 8.0 & 307.28 & 18.0 & 14.1 & 406.27 & 10.1 \\
\hline 2.0 & 74.10 & 53.1 & 8.1 & 308.90 & 12.2 & 14.2 & 407.28 & 10.1 \\
\hline 2.1 & 79.58 & 45.6 & 8.2 & 309.72 & 8.1 & 14.3 & 408.29 & 10.1 \\
\hline 2.2 & 83.21 & 38.6 & 8.3 & 310.53 & 14.9 & 14.4 & 409.30 & 10.1 \\
\hline 2.3 & 87.29 & 39.7 & 8.4 & 312.70 & 17.8 & 14.5 & 410.31 & 10.1 \\
\hline 2.4 & 91.15 & 42.8 & 8.5 & 314.10 & 12.6 & 14.6 & 411.32 & 10.1 \\
\hline 2.5 & 95.86 & 47.9 & 8.6 & 315.22 & 13.3 & 14.7 & 412.33 & 10.1 \\
\hline 2.6 & 100.73 & 46.2 & 8.7 & 316.76 & 16.6 & 14.8 & 413.34 & 10.1 \\
\hline 2.7 & 105.10 & 47.4 & 8.8 & 318.53 & 17.7 & 14.9 & 414.35 & 10.1 \\
\hline 2.8 & 110.21 & 49.4 & 8.9 & 320.30 & 21.5 & 15.0 & 415.36 & 10.1 \\
\hline 2.9 & 114.98 & 48.1 & 9.0 & 322.83 & 21.3 & 15.1 & 416.37 & 10.1 \\
\hline 3.0 & 119.84 & 46.6 & 9.1 & 324.56 & 19.0 & 15.2 & 417.38 & 10.1 \\
\hline 3.1 & 124.31 & 39.8 & 9.2 & 326.62 & 18.2 & 15.3 & 418.39 & 10.1 \\
\hline 3.2 & 127.79 & 30.2 & 9.3 & 328.20 & 16.0 & 15.4 & 419.40 & 10.1 \\
\hline 3.3 & 130.35 & 33.4 & 9.4 & 329.82 & 15.1 & 15.5 & 420.41 & 10.1 \\
\hline 3.4 & 134.48 & 44.3 & 9.5 & 331.22 & 14.2 & 15.6 & 421.43 & 10.1 \\
\hline 3.5 & 139.22 & 45.7 & 9.6 & 332.66 & 12.9 & 15.7 & 422.44 & 10.1 \\
\hline 3.6 & 143.63 & 35.9 & 9.7 & 333.81 & 13.9 & 15.8 & 423.45 & 12.1 \\
\hline 3.7 & 146.41 & 29.0 & 9.8 & 335.44 & 15.1 & 15.9 & 424.85 & 15.1 \\
\hline 3.8 & 149.43 & 29.0 & 9.9 & 336.84 & 15.2 & 16.0 & 426.48 & 16.2 \\
\hline 3.9 & 152.21 & 33.9 & 10.0 & 338.48 & 14.7 & 16.1 & 428.10 & 16.2 \\
\hline 4.0 & 156.21 & 35.3 & 10.1 & 339.78 & 12.9 & 16.2 & 429.72 & 16.2 \\
\hline 4.1 & 159.28 & 30.1 & 10.2 & 341.07 & 12.9 & 16.3 & 431.34 & 16.2 \\
\hline 4.2 & 162.24 & 31.6 & 10.3 & 342.36 & 12.5 & 16.4 & 432.96 & 16.2 \\
\hline 4.3 & 165.59 & 34.9 & 10.4 & 343.57 & 11.1 & 16.5 & 434.59 & 16.2 \\
\hline 4.4 & 169.21 & 34.3 & 10.5 & 344.59 & 10.1 & 16.6 & 436.21 & 16.2 \\
\hline 4.5 & 172.45 & 41.6 & 10.6 & 345.60 & 10.6 & 16.7 & 437.83 & 16.2 \\
\hline 4.6 & 177.53 & 52.2 & 10.7 & 346.70 & 17.2 & 16.8 & 439.45 & 16.2 \\
\hline 4.7 & 182.90 & 50.0 & 10.8 & 349.04 & 23.3 & 16.9 & 441.07 & 16.2 \\
\hline 4.8 & 187.53 & 43.4 & 10.9 & 351.37 & 22.9 & 17.0 & 442.69 & 16.2 \\
\hline 4.9 & 191.57 & 37.4 & 11.0 & 353.61 & 19.3 & 17.1 & 444.32 & 16.2 \\
\hline 5.0 & 195.01 & 29.1 & 11.1 & 355.23 & 16.2 & 17.2 & 445.94 & 16.2 \\
\hline 5.1 & 197.39 & 33.7 & 11.2 & 356.85 & 16.8 & 17.3 & 447.56 & 16.2 \\
\hline 5.2 & 201.75 & 47.0 & 11.3 & 358.59 & 17.6 & 17.4 & 449.18 & 16.2 \\
\hline 5.3 & 206.79 & 44.6 & 11.4 & 360.36 & 17.8 & 17.5 & 450.80 & 16.2 \\
\hline 5.4 & 210.67 & 43.5 & 11.5 & 362.15 & 18.0 & 17.6 & 452.43 & 16.2 \\
\hline 5.5 & 215.49 & 53.1 & 11.6 & 363.95 & 18.0 & 17.7 & 454.05 & 16.2 \\
\hline 5.6 & 221.28 & 57.0 & 11.7 & 365.75 & 18.0 & 17.8 & 455.67 & 16.2 \\
\hline 5.7 & 226.89 & 58.9 & 11.8 & 367.55 & 18.0 & 17.9 & 457.29 & 16.2 \\
\hline 5.8 & 233.07 & 59.7 & 11.9 & 369.34 & 18.0 & 18.0 & 458.91 & \\
\hline 5.9 & 238.83 & 51.4 & 12.0 & 371.14 & 18.0 & & & \\
\hline 6.0 & 243.36 & 54.9 & 12.1 & 372.94 & 18.0 & & & \\
\hline
\end{tabular}




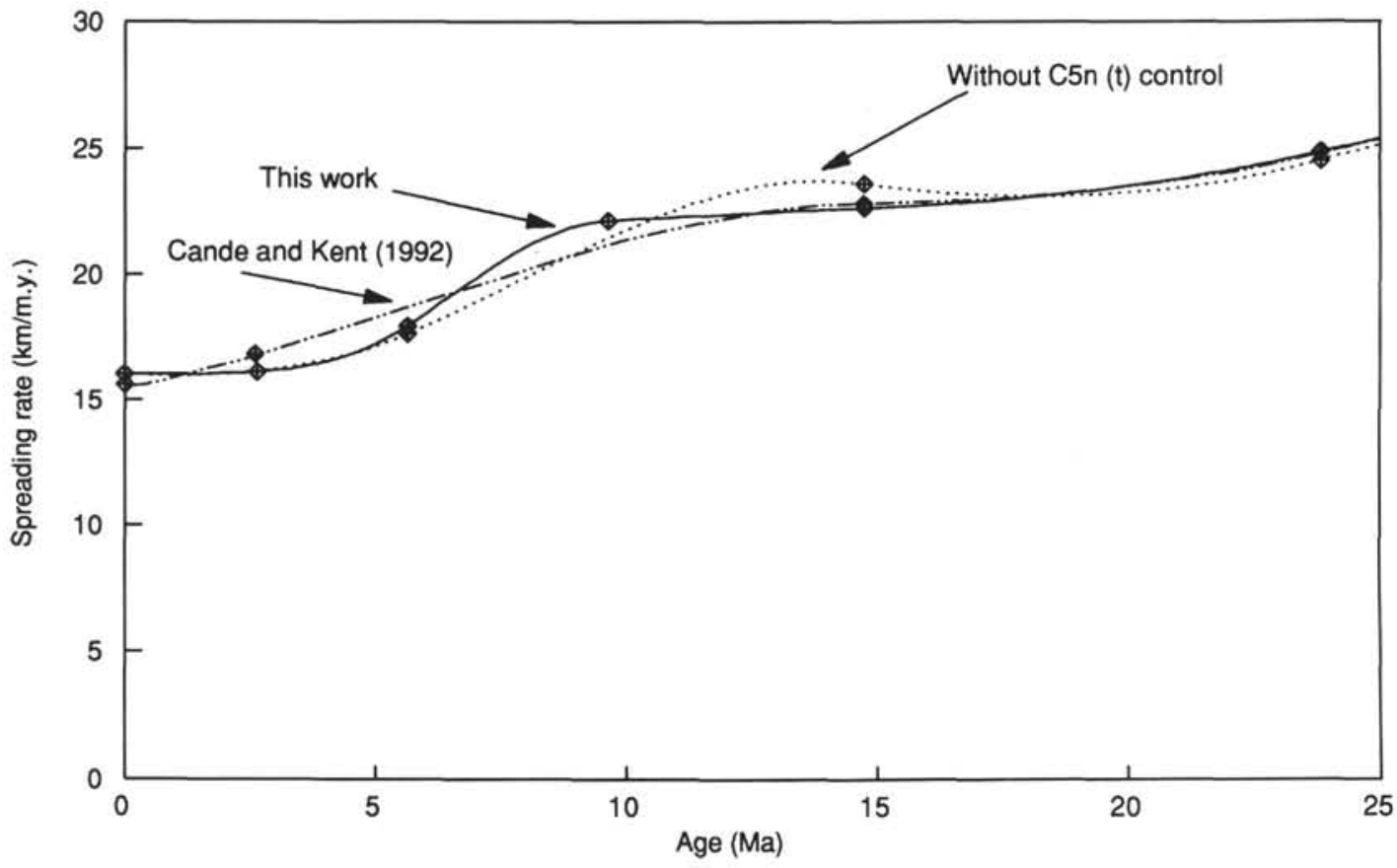

Figure 10. South Atlantic Ocean spreading rates derived by applying a cubic-spline function to the distances in CK92 (Table 2). Line A uses our calibration for C3An ( $\mathrm{t}$ ) in addition to those used in CK92; Line B, our preferred solution, includes an additional calibration at $\mathrm{C} 5 \mathrm{n} . \ln (\mathrm{t})$.

Table 21. Accumulation rates (mcd scale) estimated in overlapping 0.2 m.y. for Site 847 (from Table 4).

\begin{tabular}{|c|c|c|c|c|c|}
\hline $\begin{array}{l}\text { Age } \\
\text { (Ma) }\end{array}$ & $\begin{array}{l}\text { Depth } \\
\text { (mcd) }\end{array}$ & $\begin{array}{c}\text { Rate } \\
(\mathrm{m} / \mathrm{m} . \mathrm{y} .)\end{array}$ & $\begin{array}{l}\text { Age } \\
(\mathrm{Ma})\end{array}$ & $\begin{array}{l}\text { Depth } \\
\text { (mcd) }\end{array}$ & $\begin{array}{c}\text { Rate } \\
(\mathrm{m} / \mathrm{m} . \mathrm{y} .)\end{array}$ \\
\hline 0.0 & 0.00 & & 3.5 & 112.72 & 28.0 \\
\hline 0.1 & 3.35 & 35.3 & 3.6 & 115.62 & 29.8 \\
\hline 0.2 & 7.05 & 33.6 & 3.7 & 118.68 & 29.1 \\
\hline 0.3 & 10.07 & 32.7 & 3.8 & 121.45 & 27.6 \\
\hline 0.4 & 13.60 & 32.0 & 3.9 & 124.19 & 25.5 \\
\hline 0.5 & 16.46 & 25.7 & 4.0 & 126.55 & 23.4 \\
\hline 0.6 & 18.75 & 26.3 & 4.1 & 128.87 & 23.1 \\
\hline 0.7 & 21.71 & 29.5 & 4.3 & 133.92 & 31.3 \\
\hline 0.8 & 24.65 & 30.8 & 4.4 & 137.44 & 31.7 \\
\hline 0.9 & 27.86 & 28.9 & 4.5 & 140.27 & 43.0 \\
\hline 1.0 & 30.43 & 29.1 & 4.6 & 146.05 & 77.1 \\
\hline 1.1 & 33.69 & 34.5 & 4.7 & 155.69 & 66.8 \\
\hline 1.2 & 37.33 & 36.1 & 4.8 & 159,41 & 53.4 \\
\hline 1.3 & 40.91 & 37.1 & 4.9 & 166.37 & 50.9 \\
\hline 1.4 & 44.75 & 34.3 & 5.0 & 169.59 & 36.9 \\
\hline 1.5 & 47.76 & 29.9 & 5.1 & 173.75 & 39.8 \\
\hline 1.6 & 50.73 & 28.1 & 5.2 & 177.54 & 39.8 \\
\hline 1.7 & 53.38 & 33.0 & 5.3 & 181.70 & 39.9 \\
\hline 1.8 & 57.33 & 42.3 & 5.4 & 185.52 & 37.7 \\
\hline 2.0 & 66.05 & 41.5 & 5.5 & 189.25 & 40.5 \\
\hline 2.1 & 70.14 & 34.7 & 5.6 & 193.61 & 48.2 \\
\hline 2.2 & 73.00 & 29.4 & 5.7 & 198.89 & 45.7 \\
\hline 2.3 & 76.02 & 30.8 & 5.8 & 202.74 & 41.4 \\
\hline 2.4 & 79.15 & 35.0 & 5.9 & 207.17 & 40.9 \\
\hline 2.5 & 83.01 & 35.3 & 6.0 & 210.91 & 50.5 \\
\hline 2.6 & 86.22 & 31.4 & 6.1 & 217.27 & 64.1 \\
\hline 2.7 & 89.28 & 31.5 & 6.2 & 223.74 & 64.7 \\
\hline 2.8 & 92.53 & 30.2 & 6.3 & 230.21 & 58.3 \\
\hline 2.9 & 95.33 & 29.8 & 6.4 & 235.41 & 52.0 \\
\hline 3.0 & 98.49 & 28.4 & 6.5 & 240.61 & 52.0 \\
\hline 3.1 & 101.01 & 27.4 & 6.6 & 245.81 & 52.0 \\
\hline 3.2 & 103.96 & 30.7 & 6.7 & 251.01 & \\
\hline 3.3 & 107.16 & 30.3 & & & \\
\hline
\end{tabular}



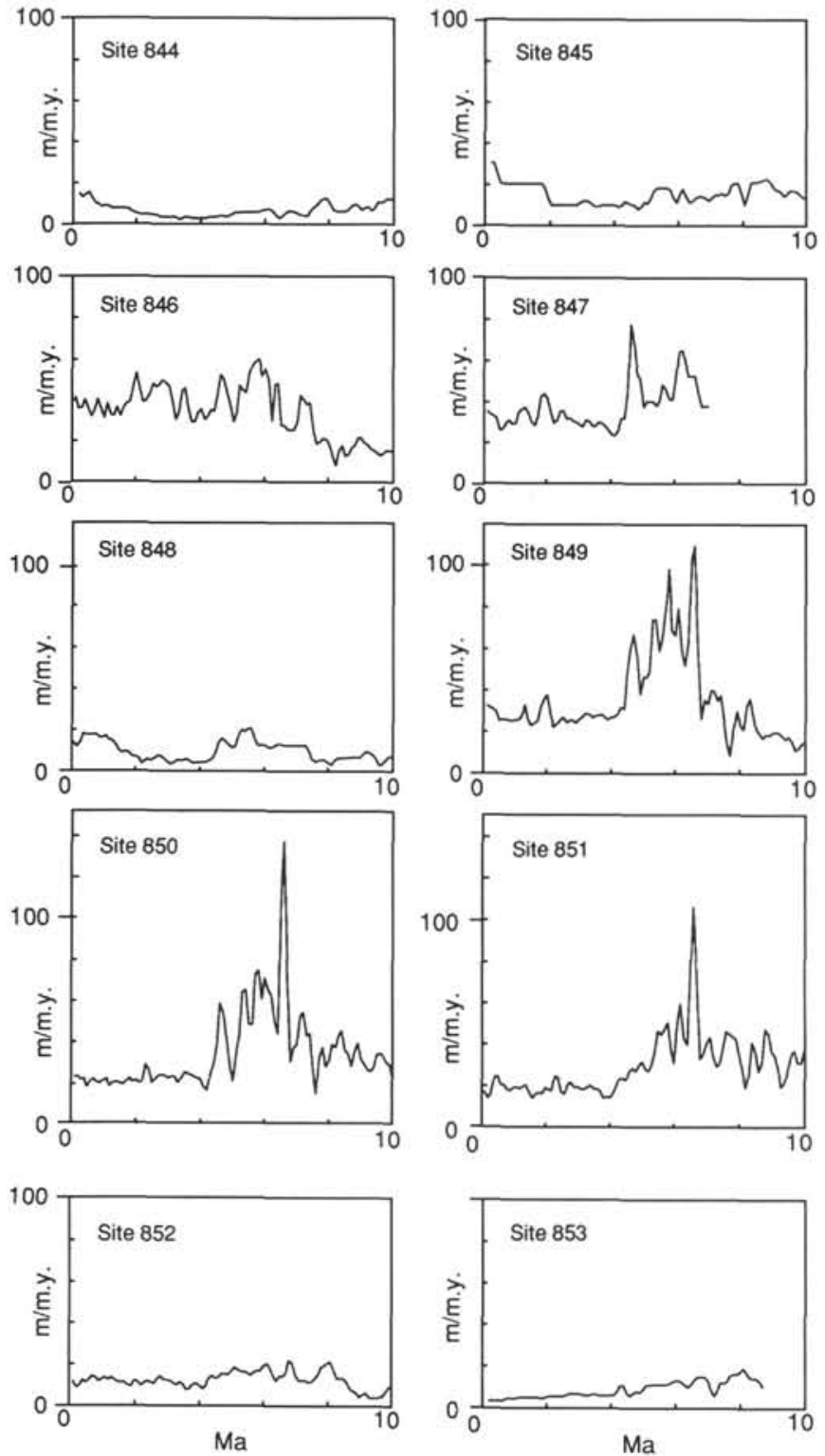

Figure 11. Sedimentation rates from Tables 18 through 28. Estimates are plotted at 0.1-m.y. intervals; each estimate plotted represents the mean for the 0.2-m.y. interval centered on that age, derived by interpolating a depth point every $0.1 \mathrm{Ma}$ from the table. Note that the values given are probably greater than the true in-situ sedimentation rates by about $10 \%$, and somewhat more in the intervals recovered by the XCB (Hagelberg et al., 1992; Fig. 3; Harris et al., this volume).
Table 22. Accumulation rates (mcd scale) estimated in overlapping 0.2 m.y. intervals for Site $\mathbf{8 4 8}$ (from Table 5).

\begin{tabular}{|c|c|c|c|c|c|}
\hline $\begin{array}{l}\text { Age } \\
\mathrm{Ma}\end{array}$ & $\begin{array}{l}\text { Depth } \\
\text { (mcd) }\end{array}$ & $\begin{array}{c}\text { Rate } \\
\mathrm{m} / \mathrm{m} . \mathrm{y} .\end{array}$ & $\begin{array}{l}\text { Age } \\
\text { Ma }\end{array}$ & $\begin{array}{l}\text { Depth } \\
\text { (mcd) }\end{array}$ & $\begin{array}{c}\text { Rate } \\
\mathrm{m} / \mathrm{m} . \mathrm{y} .\end{array}$ \\
\hline 0.0 & 0.00 & & 0.0 & 0.00 & \\
\hline 0.1 & 1.54 & 13.8 & 5.7 & 60.10 & 15.7 \\
\hline 0.2 & 2.76 & 11.8 & 5.8 & 61.34 & 12.4 \\
\hline 0.3 & 3.90 & 14.2 & 5.9 & 62.58 & 12.4 \\
\hline 0.4 & 5.60 & 18.3 & 6.0 & 63.82 & 12.4 \\
\hline 0.5 & 7.57 & 17.8 & 6.1 & 65.06 & 11.8 \\
\hline 0.6 & 9.17 & 17.6 & 6.2 & 66.19 & 11.3 \\
\hline 0.7 & 11.09 & 17.9 & 6.3 & 67.31 & 11.9 \\
\hline 0.8 & 12.74 & 17.9 & 6.4 & 68.56 & 12.8 \\
\hline 0.9 & 14.67 & 16.5 & 6.5 & 69.87 & 13.0 \\
\hline 1.0 & 16.04 & 16.4 & 6.6 & 71.16 & 12.6 \\
\hline 1.1 & 17.96 & 17.0 & 6.7 & 72.38 & 12.3 \\
\hline 1.2 & 19.44 & 15.0 & 6.8 & 73.61 & 12.3 \\
\hline 1.3 & 20.95 & 15.0 & 6.9 & 74.84 & 12.3 \\
\hline 1.4 & 22.44 & 13.1 & 7.0 & 76.07 & 12.3 \\
\hline 1.5 & 23.56 & 9.9 & 7.1 & 77.29 & 12.3 \\
\hline 1.6 & 24.42 & 8.8 & 7.2 & 78.52 & 12.3 \\
\hline 1.7 & 25.33 & 9.4 & 7.3 & 79.75 & 12.3 \\
\hline 1.8 & 26.29 & 9.2 & 7.4 & 80.98 & 10.0 \\
\hline 1.9 & 27.18 & 7.2 & 7.5 & 81.75 & 6.1 \\
\hline 2.0 & 27.72 & 7.1 & 7.6 & 82.20 & 4.6 \\
\hline 2.1 & 28.61 & 6.4 & 7.7 & 82.68 & 5.0 \\
\hline 2.2 & 29.01 & 3.7 & 7.8 & 83.21 & 5.3 \\
\hline 2.3 & 29.35 & 4.9 & 7.9 & 83.73 & 5.3 \\
\hline 2.4 & 29.98 & 5.9 & 8.0 & 84.26 & 4.1 \\
\hline 2.5 & 30.52 & 5.4 & 8.1 & 84.55 & 3.0 \\
\hline 2.6 & 31.06 & 6.3 & 8.2 & 84.86 & 4.8 \\
\hline 2.7 & 31.78 & 7.4 & 8.3 & 85.50 & 6.3 \\
\hline 2.8 & 32.55 & 7.2 & 8.4 & 86.13 & 6.3 \\
\hline 2.9 & 33.22 & 5.8 & 8.5 & 86.77 & 6.3 \\
\hline 3.0 & 33.71 & 4.3 & 8.6 & 87.40 & 6.5 \\
\hline 3.1 & 34,08 & 3.5 & 8.7 & 88.06 & 6.6 \\
\hline 3.2 & 34.41 & 4.0 & 8.8 & 88.73 & 6.7 \\
\hline 3.3 & 34.88 & 5.2 & 8.9 & 89,40 & 6.7 \\
\hline 3.4 & 35.46 & 5.0 & 9.0 & 90.06 & 6.6 \\
\hline 3.5 & 35.88 & 5.2 & 9.1 & 90.72 & 8.3 \\
\hline 3.6 & 36.50 & 5.8 & 9.2 & 91.72 & 9.6 \\
\hline 3.7 & 37.04 & 4.7 & 9.3 & 92.65 & 8.9 \\
\hline 3.8 & 37.44 & 3.9 & 9.4 & 93.50 & 8.3 \\
\hline 3.9 & 37.82 & 4.0 & 9.5 & 94.32 & 5.9 \\
\hline 4.0 & 38.24 & 4.1 & 9.6 & 94.69 & 3.2 \\
\hline 4.1 & 38.64 & 4.3 & 9.7 & 94.95 & 3.2 \\
\hline 4.2 & 39.10 & 4.3 & 9.8 & 95.32 & 5.3 \\
\hline 4.3 & 39.50 & 5.0 & 9.9 & 96.01 & 7.1 \\
\hline 4.4 & 40.10 & 6.3 & 10.0 & 96.74 & 7.2 \\
\hline 4.5 & 40.77 & 9.1 & 10.1 & 97.46 & 7.2 \\
\hline 4.6 & 41.92 & 14.1 & 10.2 & 98.18 & 7.2 \\
\hline 4.7 & 43.58 & 15.8 & 10.3 & 98.90 & 7.2 \\
\hline 4.8 & 45.08 & 14.3 & 10.4 & 99.63 & 7.2 \\
\hline 4.9 & 46.43 & 12.6 & 10.5 & 100.35 & 7.2 \\
\hline 5.0 & 47.59 & 11.2 & 10.6 & 101.07 & 7.2 \\
\hline 5.1 & 48.68 & 11.9 & 10.7 & 101.80 & 7.2 \\
\hline 5.2 & 49.97 & 16.8 & 10.8 & 102.52 & 6.6 \\
\hline 5.3 & 52.04 & 20.3 & 10.9 & 103.12 & 6.0 \\
\hline 5.4 & 54.04 & 19.4 & 11.0 & 103.71 & 6.4 \\
\hline 5.5 & 55.92 & 20.8 & 11.1 & 104.41 & 7.0 \\
\hline 5.6 & 58.20 & 20.9 & 11.2 & 105.11 & \\
\hline
\end{tabular}


Table 23. Accumulation rates (mcd scale) estimated in overlapping $\mathbf{0 . 2}$ m.y. intervals for Site 849 (from Table 6).

\begin{tabular}{|c|c|c|c|c|c|}
\hline $\begin{array}{l}\text { Age } \\
\text { (Ma) }\end{array}$ & $\begin{array}{l}\text { Depth } \\
\text { (mcd) }\end{array}$ & $\begin{array}{c}\text { Rate } \\
(\mathrm{m} / \mathrm{m} . \mathrm{y} .)\end{array}$ & $\begin{array}{l}\text { Age } \\
(\mathrm{Ma})\end{array}$ & $\begin{array}{l}\text { Depth } \\
\text { (mcd) }\end{array}$ & $\begin{array}{c}\text { Rate } \\
(\mathrm{m} / \mathrm{m} . \mathrm{y} .)\end{array}$ \\
\hline 0.0 & 0.02 & & 5.6 & 189.91 & 65.5 \\
\hline 0.1 & 3.12 & 32.9 & 5.7 & 196.45 & 79.7 \\
\hline 0.2 & 6.61 & 32.2 & 5.8 & 205.84 & 98.6 \\
\hline 0.3 & 9.55 & 31.1 & 5.9 & 216.16 & 69.2 \\
\hline 0.4 & 12.83 & 30.1 & 6.0 & 219.69 & 66.6 \\
\hline 0.5 & 15.56 & 25.7 & 6.1 & 229.49 & 79.7 \\
\hline 0.6 & 17.96 & 26.2 & 6.2 & 235.62 & 59.1 \\
\hline 0.7 & 20.81 & 26.1 & 6.3 & 241.32 & 52.3 \\
\hline 0.8 & 23.19 & 25.7 & 6.4 & 246,09 & 63.7 \\
\hline 0.9 & 25.95 & 25.5 & 6.5 & 254.05 & 102.3 \\
\hline 1.0 & 28.28 & 26.0 & 6.6 & 266.55 & 109.6 \\
\hline 1.1 & 31.15 & 25.9 & 6.7 & 275.96 & 58.1 \\
\hline 1.2 & 33.46 & 28.0 & 6.8 & 278.16 & 26.3 \\
\hline 1.3 & 36.75 & 33.1 & 6.9 & 281.22 & 35.8 \\
\hline 1.4 & 40.08 & 26.1 & 7.0 & 285.32 & 33.6 \\
\hline 1.5 & 41.97 & 23.1 & 7.1 & 287.94 & 40.5 \\
\hline 1.6 & 44.71 & 24.4 & 7.2 & 293.41 & 39.9 \\
\hline 1.7 & 46.85 & 25.6 & 7.3 & 295.91 & 35.2 \\
\hline 1.8 & 49.82 & 32.3 & 7.4 & 300.45 & 38.1 \\
\hline 1.9 & 53.31 & 36.4 & 7.5 & 303.53 & 26.8 \\
\hline 2.0 & 57.10 & 38.1 & 7.6 & 305.81 & 14.2 \\
\hline 2.1 & 60.93 & 29.4 & 7.7 & 306.37 & 8.6 \\
\hline 2.2 & 62.99 & 22.4 & 7.8 & 307.54 & 21.4 \\
\hline 2.3 & 65.42 & 23.8 & 7.9 & 310.64 & 29.9 \\
\hline 2.4 & 67.75 & 25.9 & 8.0 & 313.51 & 23.4 \\
\hline 2.5 & 70.59 & 27.3 & 8.1 & 315.33 & 21.4 \\
\hline 2.6 & 73.21 & 24.8 & 8.2 & 317.79 & 32.3 \\
\hline 2.7 & 75.55 & 25.8 & 8.3 & 321.79 & 36.5 \\
\hline 2.8 & 78.36 & 25.7 & 8.4 & 325.08 & 26.7 \\
\hline 2.9 & 80.69 & 24.5 & 8.5 & 327.14 & 21.8 \\
\hline 3.0 & 83.25 & 26.2 & 8.6 & 329.43 & 19.1 \\
\hline 3.1 & 85.93 & 27.3 & 8.7 & 330.95 & 17.1 \\
\hline 3.2 & 88.72 & 29.1 & 8.8 & 332.85 & 18.8 \\
\hline 3.3 & 91.76 & 28.7 & 8.9 & 334.71 & 18.5 \\
\hline 3.4 & 94.46 & 27.2 & 9.0 & 336.55 & 19.7 \\
\hline 3.5 & 97.21 & 27.9 & 9.1 & 338.64 & 20.1 \\
\hline 3.6 & 100.04 & 28.1 & 9.2 & 340.57 & 19.7 \\
\hline 3.7 & 102.82 & 28.6 & 9.3 & 342.59 & 18.0 \\
\hline 3.8 & 105.76 & 27.3 & 9.4 & 344.18 & 16.5 \\
\hline 3.9 & 108.28 & 26.2 & 9.5 & 345.88 & 17.5 \\
\hline 4.0 & 111.00 & 27.2 & 9.6 & 347.68 & 15.2 \\
\hline 4.1 & 113.73 & 27.4 & 9.7 & 348.92 & 11.2 \\
\hline 4.2 & 116.48 & 28.9 & 9.8 & 349.93 & 12.1 \\
\hline 4.3 & 119.50 & 32.0 & 9.9 & 351.34 & 14.5 \\
\hline 4.4 & 122.89 & 31.4 & 10.0 & 352.82 & 14.9 \\
\hline 4.5 & 125.79 & 49.2 & 10.1 & 354.31 & 14.9 \\
\hline 4.6 & 132.72 & 60.0 & 10.2 & 355.80 & 14.9 \\
\hline 4.7 & 137.78 & 67.2 & 10.3 & 357.29 & 14.9 \\
\hline 4.8 & 146.17 & 57.0 & 10.4 & 358.77 & 14.9 \\
\hline 4.9 & 149.18 & 38.5 & 10.5 & 360.26 & 17.0 \\
\hline 5.0 & 153.86 & 47.0 & 10.6 & 362.18 & 21.3 \\
\hline 5.1 & 158.57 & 46.5 & 10.7 & 364.52 & 25.9 \\
\hline 5.2 & 163.17 & 49.4 & 10.8 & 367.37 & 28.4 \\
\hline 5.3 & 168.45 & 74.3 & 10.9 & 370.21 & 28.4 \\
\hline 5.4 & 178.02 & 74.5 & 11.0 & 373.05 & 28.4 \\
\hline 5.5 & 183.35 & 59.5 & 11.1 & 375.89 & \\
\hline
\end{tabular}

Table 24. Accumulation rates (mcd scale) estimated in overlapping 0.2 m.y. intervals in Site 850 (from Table 7).

\begin{tabular}{|c|c|c|c|c|c|}
\hline $\begin{array}{l}\text { Age } \\
(\mathrm{Ma})\end{array}$ & $\begin{array}{l}\text { Depth } \\
\text { (mcd) }\end{array}$ & $\begin{array}{c}\text { Rate } \\
(\mathrm{m} / \mathrm{m} . \mathrm{y} .)\end{array}$ & $\begin{array}{l}\text { Age } \\
(\mathrm{Ma})\end{array}$ & $\begin{array}{l}\text { Depth } \\
\text { (mcd) }\end{array}$ & $\begin{array}{c}\text { Rate } \\
(\mathrm{m} / \mathrm{m} . \mathrm{y} .)\end{array}$ \\
\hline 0.0 & 0.00 & & 6.1 & 177.62 & 64.3 \\
\hline 0.1 & 1.85 & 22.0 & 6.2 & 183.10 & 61.7 \\
\hline 0.2 & 4.41 & 22.2 & 6.3 & 189.95 & 49.1 \\
\hline 0.3 & 6.29 & 20.8 & 6.4 & 192.91 & 42.5 \\
\hline 0.4 & 8.57 & 20.9 & 6.5 & 198.45 & 106.0 \\
\hline 0.5 & 10.47 & 17.1 & 6.6 & 214.12 & 135.4 \\
\hline 0.6 & 11.99 & 19.8 & 6.7 & 225.53 & 69.6 \\
\hline 0.7 & 14.44 & 20.9 & 6.8 & 228.03 & 29.2 \\
\hline 0.8 & 16.17 & 19.5 & 6.9 & 231.38 & 35.6 \\
\hline 0.9 & 18.34 & 18.9 & 7.0 & 235.15 & 37.7 \\
\hline 1.0 & 19.95 & 20.2 & 7.1 & 238.92 & 51.4 \\
\hline 1.1 & 22.37 & 20.9 & 7.2 & 245.43 & 53.4 \\
\hline 1.2 & 24.13 & 17.5 & 7.3 & 249.60 & 41.6 \\
\hline 1.3 & 25.87 & 19.2 & 7.4 & 253.75 & 42.6 \\
\hline 1.4 & 27.97 & 19.4 & 7.5 & 258.12 & 26.1 \\
\hline 1.5 & 29.75 & 19.1 & 7.6 & 258.96 & 14.3 \\
\hline 1.6 & 31.80 & 18.1 & 7.7 & 260.98 & 31.3 \\
\hline 1.7 & 33.38 & 19.5 & 7.8 & 265.23 & 35.8 \\
\hline 1.8 & 35.70 & 21.4 & 7.9 & 268.13 & 26.9 \\
\hline 1.9 & 37.65 & 19.6 & 8.0 & 270.62 & 30.4 \\
\hline 2.0 & 39.62 & 20.4 & 8.1 & 274.21 & 37.8 \\
\hline 2.1 & 41.73 & 19.1 & 8.2 & 278.17 & 36.2 \\
\hline 2.2 & 43.44 & 19.5 & 8.3 & 281.46 & 41.7 \\
\hline 2.3 & 45.63 & 27.7 & 8.4 & 286.51 & 44.3 \\
\hline 2.4 & 48.98 & 25.8 & 8.5 & 290.32 & 34.9 \\
\hline 2.5 & 50.78 & 19.2 & 8.6 & 293.49 & 33.4 \\
\hline 2.6 & 52.83 & 21.0 & 8.7 & 297.01 & 27.3 \\
\hline 2.7 & 54.99 & 22.7 & 8.8 & 298.96 & 34.0 \\
\hline 2.8 & 57.37 & 22.9 & 8.9 & 303.82 & 38.3 \\
\hline 2.9 & 59.56 & 22.4 & 9.0 & 306.62 & 30.9 \\
\hline 3.0 & 61.85 & 21.5 & 9.1 & 310.00 & 28.6 \\
\hline 3.1 & 63.86 & 22.2 & 9.2 & 312.34 & 26.0 \\
\hline 3.2 & 66.29 & 22.0 & 9.3 & 315.20 & 24.9 \\
\hline 3.3 & 68.26 & 19.1 & 9.4 & 317.31 & 26.8 \\
\hline 3.4 & 70.11 & 21.5 & 9.5 & 320.56 & 32.5 \\
\hline 3.5 & 72.57 & 24.5 & 9.6 & 323.82 & 33.3 \\
\hline 3.6 & 75.00 & 23.5 & 9.7 & 327.22 & 32.2 \\
\hline 3.7 & 77.26 & 22.9 & 9.8 & 330.27 & 29.4 \\
\hline 3.8 & 79.57 & 22.1 & 9.9 & 333.10 & 27.9 \\
\hline 3.9 & 81.69 & 21.4 & 10.0 & 335.85 & 21.7 \\
\hline 4.0 & 83.86 & 20.8 & 10.1 & 337.43 & 31.7 \\
\hline 4.1 & 85.85 & 17.4 & 10.2 & 342.18 & 40.7 \\
\hline 4.2 & 87.34 & 15.8 & 10.3 & 345.57 & 24.1 \\
\hline 4.3 & 89.00 & 23.4 & 10.4 & 347.01 & 27.2 \\
\hline 4.4 & 92.02 & 28.3 & 10.5 & 351.01 & 60.5 \\
\hline 4.5 & 94.66 & 37.0 & 10.6 & 359.10 & 63.8 \\
\hline 4.6 & 99.41 & 57.4 & 10.7 & 363.77 & 37.0 \\
\hline 4.7 & 106.15 & 52.5 & 10.8 & 366.51 & 35.1 \\
\hline 4.8 & 109.90 & 40.8 & 10.9 & 370.79 & 44.9 \\
\hline 4.9 & 114.30 & 26.5 & 11.0 & 375.48 & 46.9 \\
\hline 5.0 & 115.20 & 19.8 & 11.1 & 380.17 & 45.7 \\
\hline 5.1 & 118.26 & 33.7 & 11.2 & 384.63 & 43.1 \\
\hline 5.2 & 121.93 & 42.2 & 11.3 & 388.80 & 41.7 \\
\hline 5.3 & 126.70 & 63.2 & 11.4 & 392.97 & 24.9 \\
\hline 5.4 & 134.57 & 64.1 & 11.5 & 393.77 & 13.5 \\
\hline 5.5 & 139.53 & 47.3 & 11.6 & 395.67 & 26.1 \\
\hline 5.6 & 144.04 & 46.9 & 11.7 & 399.00 & 33.3 \\
\hline 5.7 & 148.90 & 71.6 & 11.8 & 402.32 & 33.3 \\
\hline 5.8 & 158.37 & 73.6 & 11.9 & 405.65 & \\
\hline 5.9 & 163.63 & 59.4 & & & \\
\hline 6.0 & 170.25 & 70.0 & & & \\
\hline
\end{tabular}


Table 25. Accumulation rates (mcd scale) estimated in overlapping $\mathbf{0 . 2}$ m.y. intervals for Site 851 (from Table 8).

\begin{tabular}{|c|c|c|c|c|c|}
\hline $\begin{array}{l}\text { Age } \\
\text { (Ma) }\end{array}$ & $\begin{array}{l}\text { Depth } \\
\text { (mcd) }\end{array}$ & $\begin{array}{c}\text { Rate } \\
(\mathrm{m} / \mathrm{m} . \mathrm{y} .)\end{array}$ & $\begin{array}{l}\text { Age } \\
\text { (Ma) }\end{array}$ & $\begin{array}{l}\text { Depth } \\
\text { (mcd) }\end{array}$ & $\begin{array}{c}\text { Rate } \\
(\mathrm{m} / \mathrm{m} . \mathrm{y} .)\end{array}$ \\
\hline 0.0 & 1.40 & & 6.1 & 139.10 & 50.1 \\
\hline 0.1 & 3.43 & 17.3 & 6.2 & 145.74 & 59.5 \\
\hline 0.2 & 4.86 & 13.9 & 6.3 & 151.01 & 42.7 \\
\hline 0.3 & 6.22 & 15.9 & 6.4 & 154.29 & 39.5 \\
\hline 0.4 & 8.05 & 19.3 & 6.5 & 158.90 & 74.7 \\
\hline 0.5 & 10.09 & 17.2 & 6.6 & 169.23 & 106.4 \\
\hline 0.6 & 11.49 & 18.3 & 6.7 & 180.18 & 70.4 \\
\hline 0.7 & 13.74 & 20.6 & 6.8 & 183.31 & 32.9 \\
\hline 0.8 & 15.61 & 18.2 & 6.9 & 186.76 & 35.0 \\
\hline 0.9 & 17.37 & 17.6 & 7.0 & 190.30 & 40.0 \\
\hline 1.0 & 19.13 & 19.1 & 7.1 & 194.75 & 43.0 \\
\hline 1.1 & 21.18 & 19.4 & 7.2 & 198.91 & 34.3 \\
\hline 1.2 & 23.01 & 18.5 & 7.3 & 201.60 & 29.2 \\
\hline 1.3 & 24.89 & 19.6 & 7.4 & 204.75 & 30.5 \\
\hline 1.4 & 26.94 & 19.9 & 7.5 & 207.70 & 37.2 \\
\hline 1.5 & 28.88 & 16.9 & 7.6 & 212.19 & 46.1 \\
\hline 1.6 & 30.31 & 13.9 & 7.7 & 216.92 & 45.0 \\
\hline 1.7 & 31.66 & 16.2 & 7.8 & 221.20 & 43.3 \\
\hline 1.8 & 33.55 & 16.5 & 7.9 & 225.58 & 42.4 \\
\hline 1.9 & 34.96 & 16.1 & 8.0 & 229.68 & 34.3 \\
\hline 2.0 & 36.76 & 19.1 & 8.1 & 232.43 & 27.4 \\
\hline 2.1 & 38.78 & 18.2 & 8.2 & 235.17 & 19.1 \\
\hline 2.2 & 40.40 & 17.6 & 8.3 & 236.25 & 25.3 \\
\hline 2.3 & 42.30 & 24.7 & 8.4 & 240.24 & 40.6 \\
\hline 2.4 & 45.34 & 23.8 & 8.5 & 244.38 & 35.9 \\
\hline 2.5 & 47.07 & 16.8 & 8.6 & 247.42 & 26.8 \\
\hline 2.6 & 48.70 & 16.0 & 8.7 & 249.74 & 31.3 \\
\hline 2.7 & 50.26 & 20.3 & 8.8 & 253.69 & 47.0 \\
\hline 2.8 & 52.76 & 21.7 & 8.9 & 259.14 & 44.7 \\
\hline 2.9 & 54.61 & 19.3 & 9.0 & 262.63 & 36.1 \\
\hline 3.0 & 56.62 & 19.6 & 9.1 & 266.36 & 34.5 \\
\hline 3.1 & 58.53 & 18.5 & 9.2 & 269.53 & 27.5 \\
\hline 3.2 & 60.31 & 18.2 & 9.3 & 271.86 & 19.5 \\
\hline 3.3 & 62.17 & 19.2 & 9.4 & 273.44 & 21.3 \\
\hline 3.4 & 64.16 & 19.9 & 9.5 & 276.13 & 27.7 \\
\hline 3.5 & 66.16 & 18.6 & 9.6 & 278.98 & 34.9 \\
\hline 3.6 & 67.87 & 18.8 & 9.7 & 283.11 & 36.6 \\
\hline 3.7 & 69.91 & 17.5 & 9.8 & 286.31 & 31.1 \\
\hline 3.8 & 71.38 & 14.4 & 9.9 & 289.33 & 30.6 \\
\hline 3.9 & 72.79 & 14.7 & 10.0 & 292.43 & 36.3 \\
\hline 4.0 & 74.32 & 14.3 & 10.1 & 296.59 & 43.5 \\
\hline 4.1 & 75.66 & 15.7 & 10.2 & 301.13 & 45.4 \\
\hline 4.2 & 77.47 & 19.6 & 10.3 & 305.68 & 45.4 \\
\hline 4.3 & 79.58 & 23.0 & 10.4 & 310.22 & 45.4 \\
\hline 4.4 & 82.06 & 23.6 & 10.5 & 314.76 & 45.4 \\
\hline 4.5 & 84.31 & 22.5 & 10.6 & 319.30 & 45.4 \\
\hline 4.6 & 86.56 & 26.8 & 10.7 & 323.83 & 44.4 \\
\hline 4.7 & 89.68 & 28.4 & 10.8 & 328.18 & 43.5 \\
\hline 4.8 & 92.25 & 26.8 & 10.9 & 332.52 & 43.5 \\
\hline 4.9 & 95.03 & 29.6 & 11.0 & 336.87 & 43.5 \\
\hline 5.0 & 98.16 & 31.7 & 11.1 & 341.22 & 43.5 \\
\hline 5.1 & 101.37 & 27.8 & 11.2 & 345.56 & 40.0 \\
\hline 5.2 & 103.73 & 26.8 & 11.3 & 349.21 & 36.0 \\
\hline 5.3 & 106.73 & 29.7 & 11.4 & 352.77 & 33.2 \\
\hline 5.4 & 109.66 & 36.5 & 11.5 & 355.85 & 30.2 \\
\hline 5.5 & 114.02 & 46.2 & 11.6 & 358.80 & 29.6 \\
\hline 5.6 & 118.90 & 44.3 & 11.7 & 361.76 & 29.6 \\
\hline 5.7 & 122.88 & 46.6 & 11.8 & 364.72 & 29.6 \\
\hline 5.8 & 128.22 & 50.3 & 11.9 & 367.67 & 29.6 \\
\hline 5.9 & 132.94 & 37.5 & 12.0 & 370.63 & \\
\hline 6.0 & 135.71 & 30.8 & & & \\
\hline
\end{tabular}

Table 26. Accumulation rates (mcd scale) estimated in overlapping 0.2 m.y. intervals for Site 852 (from Table 9).

\begin{tabular}{|c|c|c|c|c|c|}
\hline $\begin{array}{l}\text { Age } \\
\text { (Ma) }\end{array}$ & $\begin{array}{l}\text { Depth } \\
\text { (mcd) }\end{array}$ & $\begin{array}{c}\text { Rate } \\
(\mathrm{m} / \mathrm{m} . \mathrm{y} .)\end{array}$ & $\begin{array}{l}\text { Age } \\
\text { (Ma) }\end{array}$ & $\begin{array}{l}\text { Depth } \\
\text { (mcd) }\end{array}$ & $\begin{array}{c}\text { Rate } \\
(\mathrm{m} / \mathrm{m}, \mathrm{y} .)\end{array}$ \\
\hline 0.0 & 0.00 & & 5.5 & 66.08 & 15.3 \\
\hline 0.1 & 1.27 & 11.5 & 5.6 & 67.54 & 14.4 \\
\hline 0.2 & 2.29 & 8.9 & 5.7 & 68.96 & 15.9 \\
\hline 0.3 & 3.06 & 9.4 & 5.8 & 70.72 & 16.5 \\
\hline 0.4 & 4.16 & 12.1 & 5.9 & 72.27 & 16.4 \\
\hline 0.5 & 5.48 & 11.3 & 6.0 & 74.00 & 18.8 \\
\hline 0.6 & 6.42 & 11.9 & 6.1 & 76.03 & 19.9 \\
\hline 0.7 & 7.87 & 14.1 & 6.2 & 77.99 & 17.8 \\
\hline 0.8 & 9.23 & 13.4 & 6.3 & 79.59 & 14.0 \\
\hline 0.9 & 10.55 & 11.6 & 6.4 & 80.79 & 11.8 \\
\hline 1.0 & 11.56 & 12.6 & 6.5 & 81.95 & 13.9 \\
\hline 1.1 & 13.07 & 13.3 & 6.6 & 83.57 & 14.0 \\
\hline 1.2 & 14.21 & 12.3 & 6.7 & 84.75 & 15.2 \\
\hline 1.3 & 15.53 & 13.3 & 6.8 & 86.61 & 21.4 \\
\hline 1.4 & 16.87 & 12.0 & 6.9 & 89.03 & 20.1 \\
\hline 1.5 & 17.93 & 11.0 & 7.0 & 90.64 & 14.1 \\
\hline 1.6 & 19.08 & 11.1 & 7.1 & 91.85 & 12.1 \\
\hline 1.7 & 20.18 & 10.9 & 7.2 & 93.06 & 12.1 \\
\hline 1.8 & 21.27 & 9.7 & 7.3 & 94.27 & 12.1 \\
\hline 1.9 & 22.12 & 9.1 & 7.4 & 95.48 & 10.9 \\
\hline 2.0 & 23.09 & 11.8 & 7.5 & 96.45 & 11.4 \\
\hline 2.1 & 24.48 & 11.6 & 7.6 & 97.75 & 12.7 \\
\hline 2.2 & 25.41 & 9.4 & 7.7 & 98.99 & 14.8 \\
\hline 2.3 & 26.35 & 10.5 & 7.8 & 100.70 & 18.1 \\
\hline 2.4 & 27.52 & 11.7 & 7.9 & 102.61 & 19.1 \\
\hline 2.5 & 28.69 & 11.0 & 8.0 & 104.52 & 20.3 \\
\hline 2.6 & 29.72 & 10.3 & 8.1 & 106.67 & 20.7 \\
\hline 2.7 & 30.76 & 12.7 & 8.2 & 108.66 & 16.5 \\
\hline 2.8 & 32.27 & 14.0 & 8.3 & 109.98 & 13.1 \\
\hline 2.9 & 33.56 & 12.4 & 8.4 & 111.29 & 13.1 \\
\hline 3.0 & 34.76 & 12.0 & 8.5 & 112.60 & 13.1 \\
\hline 3.1 & 35.95 & 11.8 & 8.6 & 113.91 & 11.2 \\
\hline 3.2 & 37.13 & 10.5 & 8.7 & 114.83 & 8.2 \\
\hline 3.3 & 38.05 & 9.8 & 8.9 & 116.28 & 6.2 \\
\hline 3.4 & 39.09 & 10.9 & 9.0 & 116.80 & 4.4 \\
\hline 3.5 & 40.24 & 10.1 & 9.1 & 117.15 & 5.6 \\
\hline 3.6 & 41.11 & 7.7 & 9.2 & 117.91 & 6.2 \\
\hline 3.7 & 41.77 & 8.3 & 9.3 & 118.39 & 4.2 \\
\hline 3.8 & 42.77 & 10.0 & 9.4 & 118.75 & 3.8 \\
\hline 3.9 & 43.78 & 10.5 & 9.5 & 119.15 & 4.0 \\
\hline 4.0 & 44.87 & 9.7 & 9.6 & 119.54 & 4.0 \\
\hline 4.1 & 45.72 & 8.1 & 9.7 & 119.96 & 4.5 \\
\hline 4.2 & 46.48 & 9.2 & 9.8 & 120.44 & 6.6 \\
\hline 4.3 & 47.56 & 12.9 & 9.9 & 121.28 & 8.8 \\
\hline 4.4 & 49.06 & 14.3 & 10.0 & 122.20 & 9.5 \\
\hline 4.5 & 50.42 & 13.0 & 10.1 & 123.17 & 9.8 \\
\hline 4.6 & 51.67 & 13.6 & 10.2 & 124.16 & 9.9 \\
\hline 4.7 & 53.14 & 15.3 & 10.3 & 125.15 & 9.9 \\
\hline 4.8 & 54.72 & 15.0 & 10.4 & 126.14 & 9.9 \\
\hline 4.9 & 56.14 & 14.7 & 10.5 & 127.13 & 8.8 \\
\hline 5.0 & 57.66 & 16.5 & 10.6 & 127.90 & 4.7 \\
\hline 5.1 & 59.43 & 18.0 & 10.7 & 128.08 & 1.7 \\
\hline 5.2 & 61.25 & 17.0 & 10.8 & 128.25 & \\
\hline 5.3 & 62.83 & 16.1 & & & \\
\hline
\end{tabular}


Table 27. Accumulation rates (mcd scale) estimated in overlapping $\mathbf{0 . 2}$ m.y. intervals for Site 853 (from Table 10).

\begin{tabular}{|c|c|c|c|c|c|}
\hline $\begin{array}{l}\text { Age } \\
\text { (Ma) }\end{array}$ & $\begin{array}{l}\text { Depth } \\
\text { (mcd) }\end{array}$ & $\begin{array}{c}\text { Rate } \\
(\mathrm{m} / \mathrm{m} . \mathrm{y} .)\end{array}$ & $\begin{array}{l}\text { Age } \\
\text { (Ma) }\end{array}$ & $\begin{array}{l}\text { Depth } \\
\text { (mcd) }\end{array}$ & $\begin{array}{c}\text { Rate } \\
(\mathrm{m} / \mathrm{m} . \mathrm{y} .)\end{array}$ \\
\hline 0.0 & 0.22 & & 4.4 & 24.57 & 10.7 \\
\hline 0.1 & 0.55 & 3.3 & 4.5 & 25.48 & 7.0 \\
\hline 0.2 & 0.88 & 3.3 & 4.6 & 25.98 & 5.9 \\
\hline 0.3 & 1.20 & 3.3 & 4.7 & 26.66 & 7.1 \\
\hline 0.4 & 1.53 & 3.3 & 4.8 & 27.40 & 7.9 \\
\hline 0.5 & 1.86 & 3.3 & 4.9 & 28.25 & 7.7 \\
\hline 0.6 & 2.20 & 3.5 & 5.0 & 28.93 & 8.7 \\
\hline 0.7 & 2.56 & 4.2 & 5.1 & 29.99 & 10.7 \\
\hline 0.8 & 3.04 & 4.5 & 5.2 & 31.07 & 10.9 \\
\hline 0.9 & 3.46 & 4.3 & 5.3 & 32.17 & 11.1 \\
\hline 1.0 & 3.89 & 4.4 & 5.4 & 33.28 & 11.1 \\
\hline 1.1 & 4.34 & 4.8 & 5.5 & 34.39 & 11.1 \\
\hline 1.2 & 4.86 & 5.2 & 5.6 & 35.51 & 11.1 \\
\hline 1.3 & 5.38 & 5.2 & 5.7 & 36.62 & 11.1 \\
\hline 1.4 & 5.89 & 5.2 & 5.8 & 37.73 & 11.4 \\
\hline 1.5 & 6.41 & 5.2 & 5.9 & 38.90 & 12.3 \\
\hline 1.6 & 6.92 & 5.2 & 6.0 & 40.20 & 13.1 \\
\hline 1.7 & 7.44 & 5.0 & 6.1 & 41.51 & 13.1 \\
\hline 1.8 & 7.93 & 4.6 & 6.2 & 42.82 & 12.4 \\
\hline 1.9 & 8.36 & 4.6 & 6.3 & 43.99 & 10.8 \\
\hline 2.0 & 8.85 & 5.2 & 6.4 & 44.99 & 10.0 \\
\hline 2.1 & 9.40 & 5.6 & 6.5 & 45.98 & 11.1 \\
\hline 2.2 & 9.96 & 5.6 & 6.6 & 47.20 & 13.5 \\
\hline 2.3 & 10.51 & 5.6 & 6.7 & 48.67 & 14.7 \\
\hline 2.4 & 11.07 & 5.6 & 6.8 & 50.15 & 14.7 \\
\hline 2.5 & 11.62 & 5.6 & 6.9 & 51.62 & 14.7 \\
\hline 2.6 & 12.18 & 6.1 & 7.0 & 53.10 & 13.5 \\
\hline 2.7 & 12.85 & 6.7 & 7.1 & 54.32 & 9.0 \\
\hline 2.8 & 13.52 & 6.7 & 7.2 & 54.89 & 5.7 \\
\hline 2.9 & 14.20 & 6.7 & 7.3 & 55.46 & 8.2 \\
\hline 3.0 & 14.87 & 6.8 & 7.4 & 56.52 & 11.9 \\
\hline 3.1 & 15.55 & 6.7 & 7.5 & 57.85 & 11.8 \\
\hline 3.2 & 16.20 & 6.0 & 7.6 & 58.88 & 12.5 \\
\hline 3.3 & 16.76 & 6.0 & 7.7 & 60.36 & 15.4 \\
\hline 3.4 & 17.39 & 6.6 & 7.8 & 61.96 & 16.0 \\
\hline 3.5 & 18.07 & 6.7 & 7.9 & 63.56 & 16.0 \\
\hline 3.6 & 18.74 & 6.4 & 8.0 & 65.17 & 17.2 \\
\hline 3.7 & 19.35 & 6.1 & 8.1 & 67.00 & 18.7 \\
\hline 3.8 & 19.96 & 6.0 & 8.2 & 68.91 & 16.7 \\
\hline 3.9 & 20.56 & 6.0 & 8.3 & 70.34 & 14.2 \\
\hline 4.0 & 21.16 & 6.0 & 8.4 & 71.75 & 14.1 \\
\hline 4.1 & 21.77 & 6.2 & 8.5 & 73.16 & 14.1 \\
\hline 4.2 & 22.41 & $\begin{array}{r}7.9 \\
\end{array}$ & 8.6 & 74.57 & \\
\hline 4.3 & 23.34 & 10.8 & & & \\
\hline
\end{tabular}

Table 28. Accumulation rates (mcd scale) estimated in overlapping 0.2 m.y. intervals for Site 954 (from Table 11).

\begin{tabular}{|c|c|c|c|c|c|}
\hline $\begin{array}{l}\text { Age } \\
\text { (Ma) }\end{array}$ & $\begin{array}{l}\text { Depth } \\
\text { (mcd) }\end{array}$ & $\begin{array}{c}\text { Rate } \\
(\mathrm{m} / \mathrm{m} . \mathrm{y} .)\end{array}$ & $\begin{array}{l}\text { Age } \\
\text { (Ma) }\end{array}$ & $\begin{array}{l}\text { Depth } \\
\text { (mcd) }\end{array}$ & $\begin{array}{c}\text { Rate } \\
(\mathrm{m} / \mathrm{m} . \mathrm{y} .)\end{array}$ \\
\hline 0.0 & 0.00 & & 4.8 & 19.71 & 0.2 \\
\hline 0.1 & 0.52 & 5.2 & 4.9 & 19.73 & 0.2 \\
\hline 0.2 & 1.05 & 5.2 & 5.0 & 19.75 & 0.2 \\
\hline 0.3 & 1.57 & 5.2 & 5.1 & 19.77 & 0.2 \\
\hline 0.4 & 2.09 & 5.2 & 5.2 & 19.79 & 0.2 \\
\hline 0.5 & 2.62 & 5.2 & 5.3 & 19.81 & 0.2 \\
\hline 0.6 & 3.14 & 5.2 & 5.4 & 19.83 & 0.2 \\
\hline 0.7 & 3.66 & 5.3 & 5.5 & 19.85 & 0.2 \\
\hline 0.8 & 4.20 & 5.7 & 5.6 & 19.87 & 0.2 \\
\hline 0.9 & 4.81 & 6.0 & 5.7 & 19.89 & 0.2 \\
\hline 1.0 & 5.41 & 6.1 & 5.8 & 19.91 & 0.8 \\
\hline 1.1 & 6.03 & 6.5 & 5.9 & 20.05 & 3.1 \\
\hline 1.2 & 6.71 & 6.8 & 6.0 & 20.53 & 4.8 \\
\hline 1.3 & 7.39 & 6.8 & 6.1 & 21.00 & 5.2 \\
\hline 1.4 & 8.08 & 6.8 & 6.2 & 21.58 & 5.3 \\
\hline 1.5 & 8.76 & 6.8 & 6.3 & 22.07 & 4.4 \\
\hline 1.6 & 9.45 & 6.8 & 6.4 & 22.45 & 3.7 \\
\hline 1.7 & 10.13 & 6.5 & 6.5 & 22.82 & 4.3 \\
\hline 1.8 & 10.76 & 5.5 & 6.6 & 23.31 & 5.5 \\
\hline 2.0 & 11.88 & 7.2 & 6.7 & 23.92 & 6.2 \\
\hline 2.1 & 12.68 & 8.0 & 6.8 & 24.54 & 6.2 \\
\hline 2.2 & 13.48 & 8.0 & 6.9 & 25.15 & 9.7 \\
\hline 2.3 & 14.28 & 8.0 & 7.0 & 26.49 & 13.4 \\
\hline 2.4 & 15.08 & 8.0 & 7.1 & 27.83 & 11.4 \\
\hline 2.5 & 15.88 & 8.0 & 7.2 & 28.78 & 9.4 \\
\hline 2.6 & 16.68 & 5.1 & 7.3 & 29.72 & 9.4 \\
\hline 2.7 & 16.90 & 5.2 & 7.4 & 30.66 & 12.3 \\
\hline 2.8 & 17.12 & 2.2 & 7.5 & 32.18 & 12.3 \\
\hline 2.9 & 17.34 & 2.2 & 7.6 & 33.12 & 11.8 \\
\hline 3.0 & 17.56 & 2.1 & 7.7 & 34.54 & 15.1 \\
\hline 3.1 & 17.76 & 1.8 & 7.8 & 36.14 & 16.0 \\
\hline 3.2 & 17.92 & 1.6 & 7.9 & 37.75 & 16.0 \\
\hline 3.3 & 18.08 & 2.2 & 8.0 & 39.35 & 15.1 \\
\hline 3.4 & 18.36 & 3.1 & 8.1 & 40.76 & 14.3 \\
\hline 3.5 & 18.70 & 3.4 & 8.2 & 42.21 & 9.4 \\
\hline 3.6 & 19.04 & 3.3 & 8.3 & 42.63 & 3.8 \\
\hline 3.7 & 19.37 & 2.3 & 8.4 & 42.98 & 3.5 \\
\hline 3.8 & 19.51 & 0.8 & 8.5 & 43.32 & 3.5 \\
\hline 3.9 & 19.53 & 0.2 & 8.6 & 43.67 & 4.1 \\
\hline 4.0 & 19.55 & 0.2 & 8.7 & 44.14 & 4.9 \\
\hline 4.1 & 19.57 & 0.2 & 8.9 & 45.17 & 5.3 \\
\hline 4.3 & 19.61 & 0.2 & 9.0 & 45.72 & 5.7 \\
\hline 4.4 & 19.63 & 0.2 & 9.1 & 46.30 & 7.1 \\
\hline 4.5 & 19.65 & 0.2 & 9.2 & 47.15 & 7.5 \\
\hline 4.3 & 19.61 & 0.2 & 9.3 & 47.80 & 6.2 \\
\hline 4.4 & 19.63 & 0.2 & 9.4 & 48.38 & 6.2 \\
\hline 4.5 & 19.65 & 0.2 & 9.5 & 49.04 & 6.5 \\
\hline 4.6 & 19.67 & 0.2 & 9.6 & 49.67 & \\
\hline
\end{tabular}

